\title{
BLOW-UP PROFILE FOR THE COMPLEX-VALUED SEMILINEAR WAVE EQUATION
}

\author{
ASMA AZAIEZ
}

\begin{abstract}
In this paper, we consider a blow-up solution for the complexvalued semilinear wave equation with power non-linearity in one space dimension. We first characterize all the solutions of the associated stationary problem as a two-parameter family. Then, we use a dynamical system formulation to show that the solution in self-similar variables approaches some particular stationary one in the energy norm, in the non-characteristic case. This gives the blow-up profile for the original equation in the non-characteristic case. Our analysis is not just a simple adaptation of the already handled real case. In particular, there is one more neutral-direction in our problem, which we control thanks to a modulation technique.
\end{abstract}

\section{INTRODUCTION}

1.1. The problem and known results. We consider the following complexvalued one-dimensional semilinear wave equation:

$$
\left\{\begin{array}{l}
\partial_{t}^{2} u=\partial_{x}^{2} u+|u|^{p-1} u \\
u(0)=u_{0} \text { and } u_{t}(0)=u_{1}
\end{array}\right.
$$

where $u(t): x \in \mathbb{R} \rightarrow u(x, t) \in \mathbb{C}, p>1, u_{0} \in H_{l o c, u}^{1}$ and $u_{1} \in L_{l o c, u}^{2}$, with

$$
\|v\|_{L_{l o c, u}^{2}}^{2}=\sup _{a \in \mathbb{R}} \int_{|x-a|<1}|v(x)|^{2} d x \text { and }\|v\|_{H_{l o c, u}^{1}}^{2}=\|v\|_{L_{l o c, u}^{2}}^{2}+\|\nabla v\|_{L_{l o c, u}^{2}}^{2} .
$$

The Cauchy problem for equation (11) in the space $H_{l o c, u}^{1} \times L_{l o c, u}^{2}$ follows from the finite speed of propagation and the wellposedness in $H^{1} \times L^{2}$. See for instance Ginibre, Soffer and Velo [15, Ginibre and Velo [16, and Lindblad and Sogge 29] (for the local in time wellposedness in $H^{1} \times L^{2}$ ). The existence of blow-up solutions for equation (11) is a consequence of the finite speed of propagation and ODE techniques (see for example Levine [28] and Antonini and Merle [3]). More blow-up results can be found in Caffarelli and Friedman 4, Alinhac 1 and 2, Kichenassamy and Littman [23, 22], and Shatah and Struwe [42].

The real case (in one space dimension) has been understood completely in a series of papers by Merle and Zaag [33, 34], 37] and [38] and in Côte and Zaag [5] (see also the note [35]). Some of those results have been extended to higher

Received by the editors June 19, 2013 and, in revised form, October 25, 2013 and November 30, 2013 .

2010 Mathematics Subject Classification. Primary 35L05, 35L81, 35B44, 39B32, 35B40, 34K21, 35B35.

Key words and phrases. Wave equation, blow-up profile, stationary solution, modulation technique, complex-valued PDE. 
dimensions for conformal or subconformal $p$ :

$$
1<p \leq p_{c} \equiv 1+\frac{4}{N-1},
$$

under radial symmetry outside the origin in [36]. For non-radial solutions, we would like to mention [30] and 31] where the blow-up rate was obtained. We also mention the recent contribution of [39] and [40] where the blow-up behavior is given, together with some stability results.

Considering the behavior of radial solutions at the origin, Donninger and Schörkhuber [8] were able to prove the stability of the space-independent solution (i.e. the solution of the associated ODE $u^{\prime \prime}=u^{p}$ ) with respect to perturbation in the initial data. Willing to be as exhaustive as possible in our bibliography about the blow-up question for equation (11), we would like to mention some blow-up results in the superconformal, Sobolev critical and supercritical ranges for equation (11).

When

$$
N \geq 2 \text { and } p_{c}<p<p_{s} \equiv \frac{N+2}{N-2},
$$

Killip, Stoval and Visan found in 24 an upper bound on the blow-up rate. That bound is larger than the solution of the associated ODE $u^{\prime \prime}=u^{p}$, and is therefore thought to be non-optimal. In [17] Hamza and Zaag gives a different proof of the results of 24, improving some of their estimates.

When

$$
N \geq 3 \text { and } p=p_{s},
$$

equation (1) has attracted much interest. Many authors addressed the question of obtaining sufficient conditions for scattering and blow-up through energy estimates, in relation to the ground state (see Kenig and Merle [20] and Duyckaerts and Merle [13]). Furthermore, dynamics around the soliton were studied: see Krieger and Schlag [25] and Krieger, Nakanishi and Schlag [26] and 27]. There are also some remarkable classification theorems by Duyckaerts, Kenig and Merle [9], 11, 10] and 12. Concerning the blow-up behavior, we would like to mention that Donninger, Huang, Krieger and Schlag prove in [6] the existence of so-called "exotic" blow-up solutions when $N=3$, whose blow-up rate oscillates between several pure-power laws.

When

$$
N \geq 3 \text { and } p>p_{s},
$$

much less is known. We would just like to mention that the stability result of Donninger and Schörkhuber proved in [7] for the superconformal range does hold in the Sobolev supercritical range too, at least when $N=3$. There is also a remarkable result by Kenig and Merle [21] on the dynamics of solutions with some compactness property.

In this work, our aim is to study the profile of blow-up solutions in the complex case of equation (11). In particular, relying on the work of Merle and Zaag in 33, we give a trapping result near the set of non-zero stationary solutions in self-similar variables. This study is far from being trivial since the complex structure introduces an additional zero eigenfunction in the linearized equation around the expected profile, and also because of the coupling between the real and the imaginary parts.

If $u$ is a blow-up solution of (1), we define (see for example Alinhac [1]) a continuous curve $\Gamma$ as the graph of a function $x \mapsto T(x)$ such that the domain of definition 
of $u$ (or the maximal influence domain of $u$ ) is

$$
D_{u}=\{(x, t) \mid t<T(x)\} .
$$

From the finite speed of propagation, $T$ is a 1-Lipschitz function. The time $\check{T}=\inf _{x \in \mathbb{R}} T(x)$ and the graph $\Gamma$ are called (respectively) the blow-up time and the blow-up graph of $u$.

Let us introduce the following non-degeneracy condition for $\Gamma$. If we introduce for all $x \in \mathbb{R}, t \leq T(x)$ and $\delta>0$, the cone

$$
\mathcal{C}_{x, t, \delta}=\{(\xi, \tau) \neq(x, t)|0 \leq \tau \leq t-\delta| \xi-x \mid\},
$$

then our non-degeneracy condition is the following: $x_{0}$ is a non-characteristic point if

$$
\exists \delta=\delta\left(x_{0}\right) \in(0,1) \text { such that } u \text { is defined on } \mathcal{C}_{x_{0}, T\left(x_{0}\right), \delta_{0}} .
$$

If condition (3) is not true, then we call $x_{0}$ a characteristic point. Already when $u$ is real-valued, we know from [37] and [5] that there exist blow-up solutions with characteristic points.

Given some $x_{0} \in \mathbb{R}$, we introduce the following self-similar change of variables:

$$
w_{x_{0}}(y, s)=\left(T\left(x_{0}\right)-t\right)^{\frac{2}{p-1}} u(x, t), \quad y=\frac{x-x_{0}}{T\left(x_{0}\right)-t}, \quad s=-\log \left(T\left(x_{0}\right)-t\right) .
$$

This change of variables transforms the backward light cone with vertex $\left(x_{0}, T\left(x_{0}\right)\right)$ into the infinite cylinder $(y, s) \in(-1,1) \times\left[-\log T\left(x_{0}\right),+\infty\right)$. The function $w_{x_{0}}$ (we write $w$ for simplicity) satisfies the following equation for all $|y|<1$ and $s \geq-\log T\left(x_{0}\right):$

$$
\partial_{s}^{2} w=\mathcal{L} w-\frac{2(p+1)}{(p-1)^{2}} w+|w|^{p-1} w-\frac{p+3}{p-1} \partial_{s} w-2 y \partial_{y s} w,
$$

where

$$
\mathcal{L} w=\frac{1}{\rho} \partial_{y}\left(\rho\left(1-y^{2}\right) \partial_{y} w\right) \text { and } \rho(y)=\left(1-y^{2}\right)^{\frac{2}{p-1}} .
$$

This equation will be studied in the space

$\mathcal{H}=\left\{q \in H_{\text {loc }}^{1} \times L_{\text {loc }}^{2}((-1,1), \mathbb{C}) \mid\|q\|_{\mathcal{H}}^{2} \equiv \int_{-1}^{1}\left(\left|q_{1}\right|^{2}+\left|q_{1}^{\prime}\right|^{2}\left(1-y^{2}\right)+\left|q_{2}\right|^{2}\right) \rho d y<+\infty\right\}$,

which is the energy space for $w$. Note that $\mathcal{H}=\mathcal{H}_{0} \times L_{\rho}^{2}$ where

(8) $\mathcal{H}_{0}=\left\{r \in H_{l o c}^{1}((-1,1), \mathbb{C}) \mid\|r\|_{\mathcal{H}_{0}}^{2} \equiv \int_{-1}^{1}\left(\left|r^{\prime}\right|^{2}\left(1-y^{2}\right)+|r|^{2}\right) \rho d y<+\infty\right\}$.

Let us define

$$
E\left(w, \partial_{s} w\right)=\int_{-1}^{1}\left(\frac{1}{2}\left|\partial_{s} w\right|^{2}+\frac{1}{2}\left|\partial_{y} w\right|^{2}\left(1-y^{2}\right)+\frac{p+1}{(p-1)^{2}}|w|^{2}-\frac{1}{p+1}|w|^{p+1}\right) \rho d y .
$$

By the argument of Antonini and Merle [3, which works straightforwardly in the complex case, we see that $E$ is a Lyapunov functional for equation (5). Similarly, some arguments of the real case can be adapted with no problems to the complexcase, others cannot. As a matter of fact, the derivation of the blow-up rate works as in the real case whereas the convergence to the profile needs intricate estimates, 
and this is the goal of our paper. Let us first briefly state the result for the blow-up rate, then focus on the convergence question.

1.2. Blow-up rate. Only in this subsection, the space dimension will be extended to any $N \geq 1$. We assume in addition that $p$ is conformal or subconformal (see (2)). We recall that for the real case of equation (11), Merle and Zaag determined in [30. and [31] the blow-up rate for (11) in the region $\{(x, t) \mid t<\check{T}\}$ in a first step. Then in [32, they extended their result to the whole domain of definition $\{(x, t) \mid t<T(x)\}$. In the following, we give the growth estimate near the blow-up surface for solutions of equation (11).

Proposition 1 (Growth estimate near the blow-up surface for solutions of equation (10). If $u$ is a solution of (11) with blow-up surface $\Gamma:\{x \rightarrow T(x)\}$, and if $x_{0} \in \mathbb{R}^{N}$ is non-characteristic (in the sense of (3)), then:

(i) (Uniform bounds on $w$ ) For all $s \geq-\log \frac{T\left(x_{0}\right)}{4}$ :

$$
0<\epsilon_{0}(N, p) \leq\left\|w_{x_{0}}(s)\right\|_{H^{1}(B)}+\left\|\partial_{s} w_{x_{0}}(s)\right\|_{L^{2}(B)} \leq K .
$$

(ii) (Uniform bounds on $u$ ) For all $t \in\left[\frac{3}{4} T\left(x_{0}\right), T\left(x_{0}\right)\right.$ ):

$$
\begin{aligned}
& 0<\epsilon_{0}(N, p) \leq\left(T\left(x_{0}\right)-t\right)^{\frac{2}{p-1}} \frac{\|u(t)\|_{L^{2}\left(B\left(x_{0}, T\left(x_{0}\right)-t\right)\right)}}{\left(T\left(x_{0}\right)-t\right)^{N / 2}} \\
& +\left(T\left(x_{0}\right)-t\right)^{\frac{2}{p-1}+1}\left(\frac{\left\|\partial_{t} u(t)\right\|_{L^{2}\left(B\left(x_{0}, T\left(x_{0}\right)-t\right)\right)}}{\left(T\left(x_{0}\right)-t\right)^{N / 2}}+\frac{\|\nabla u(t)\|_{L^{2}\left(B\left(x_{0}, T\left(x_{0}\right)-t\right)\right)}}{\left(T\left(x_{0}\right)-t\right)^{N / 2}}\right) \leq K,
\end{aligned}
$$

where the constant $K$ depends only on $N, p$, and on an upper bound on $T\left(x_{0}\right)$, $1 / T\left(x_{0}\right), \delta_{0}\left(x_{0}\right)$ and the initial data in $H_{l o c, u}^{1} \times L_{l o c, u}^{2}$.

Proof. The idea of the proof is the same as in 32. For the sake of completeness, we give a sketch of the proof in Appendix A.

With the bounds in Proposition 1, we ask the question of compactness of the solution and the question of convergence of $w$ to a stationary solution of (5).

1.3. Blow-up profile. From now on, we assume again that

$$
N=1 \text {. }
$$

This subsection is the heart of our work. Indeed, unlike for the blow-up rate, it is not a simple adaptation of the real case. It involves many new ideas of ours. The first step towards the determination of the blow-up profile is to characterize all stationary solutions in $\mathcal{H}_{0}$ of equation (5).

Proposition 2 (Characterization of all stationary solution of equation (5) in $\mathcal{H}_{0}$ ). (i) Consider $w \in \mathcal{H}_{0}$ a stationary solution of (5). Then, either $w \equiv 0$ or there exist $\delta \in(-1,1)$ and $\theta \in \mathbb{R}$ such that $w(y)=e^{i \theta} \kappa(\delta, y)$ where

$$
\forall(\delta, y) \in(-1,1)^{2}, \kappa(\delta, y)=\kappa_{0} \frac{\left(1-\delta^{2}\right)^{\frac{1}{p-1}}}{(1+\delta y)^{\frac{2}{p-1}}} \text { and } \kappa_{0}=\left(\frac{2(p+1)}{(p-1)^{2}}\right)^{\frac{1}{p-1}} .
$$

(ii) It holds that

$$
E(0,0)=0 \text { and } \forall \delta \in(-1,1), \forall \theta \in \mathbb{R}, E\left(e^{i \theta} \kappa(\delta, \cdot), 0\right)=E\left(\kappa_{0}, 0\right)>0,
$$

where $E$ is given by (9). 
Remark. Note that the proof of this proposition is very different from the real case. Indeed, in the real case, the result follows from a transformation of the hyperbolic plane, which gives nothing in the complex case. We succeed in proving the result relying on ODE techniques for complex-valued equation.

Remark. Unlike the real case where the set of stationary solutions is made of three connected components: $\{0\},\{+\kappa(\delta, y)\}$ and $\{-\kappa(\delta, y)\}$, we have only two connected components: $\{0\}$ and $\left\{e^{i \theta} \kappa(\delta, y)|\theta \in \mathbb{R},| \delta \mid<1\right\}$. This is one of the novelties of our approach. Indeed, here we need a modulation technique to control the parameter $\theta$ which may take any real value, unlike the real case, where it was equal to $k \pi$ only.

The second step is the same as in the real case, and involves no novelty on our behalf. It uses the Lyapunov functional to show that when $x_{0}$ is non-characteristic, then $w_{x_{0}}$ approaches the set of non-zero stationary solutions. This is the result:

Proposition 3 (Approaching the set of non-zero stationary solutions near a noncharacteristic point). Consider $u$ a solution of (10) with blow-up curve $\Gamma:\{x \rightarrow$ $T(x)\}$. If $x_{0} \in \mathbb{R}$ is non-characteristic, then:

(A.i) $\inf _{\{\theta \in \mathbb{R},|\delta|<1\}}\left\|w_{x_{0}}(\cdot, s)-e^{i \theta} \kappa(\delta, \cdot)\right\|_{H^{1}(-1,1)}+\left\|\partial_{s} w_{x_{0}}\right\|_{L^{2}(-1,1)} \rightarrow 0$ as $s \rightarrow \infty$. (A.ii) $E\left(w_{x_{0}}(s), \partial_{s} w_{x_{0}}(s)\right) \rightarrow E\left(\kappa_{0}, 0\right)$ as $s \rightarrow \infty$.

Proof. The idea of the proof is the same as in [33. For the sake of completeness, we give a sketch of the proof in Appendix A.

From this result, we wonder whether $\theta$ and $\delta$ have limits as $s \rightarrow \infty$; in other words, whether $w_{x_{0}}(\cdot, s)$ converges to some $e^{i \theta_{\infty}\left(x_{0}\right)} \kappa\left(\delta_{\infty}\left(x_{0}\right)\right)$ for some $\theta_{\infty}\left(x_{0}\right) \in$ $\mathbb{R}$ and $\left|\delta_{\infty}\left(x_{0}\right)\right|<1$. The answer is in fact positive, as one sees in Theorem 5 below that the following trapping result of solutions of equation (5) near non-zero stationary solutions is a major tool towards this result.

In the following, we consider $w \in C\left(\left[s^{*}, \infty\right), \mathcal{H}\right)$ and show that if $w\left(s^{*}\right)$ is close enough to some non-zero stationary solution and satisfies an energy barrier, then $w(s)$ converges to a neighboring stationary solution as $s \rightarrow \infty$.

Theorem 4 (Trapping near the set of non-zero stationary solutions of (5)). There exist positive $\epsilon_{0}, \mu_{0}$ and $C_{0}$ such that if $w \in C\left(\left[s^{*}, \infty\right), \mathcal{H}\right)$ for some $s^{*} \in \mathbb{R}$ is a solution of equation (5) such that

$$
\forall s \geq s^{*}, E\left(w(s), \partial_{s} w(s)\right) \geq E\left(\kappa_{0}, 0\right),
$$

and

$$
\left\|\left(\begin{array}{c}
w\left(s^{*}\right) \\
\partial_{s} w\left(s^{*}\right)
\end{array}\right)-e^{i \theta^{*}}\left(\begin{array}{c}
\kappa\left(\delta^{*}, \cdot\right) \\
0
\end{array}\right)\right\|_{\mathcal{H}} \leq \epsilon^{*}
$$

for some $\delta^{*} \in(-1,1), \theta^{*} \in \mathbb{R}$ and $\epsilon^{*} \in\left(0, \epsilon_{0}\right]$, then there exists $\delta_{\infty} \in(-1,1)$ and $\theta_{\infty} \in \mathbb{R}$ such that

$$
\left|\operatorname{arctanh} \delta_{\infty}-\operatorname{arctanh} \delta^{*}\right|+\left|\theta_{\infty}-\theta^{*}\right| \leq C_{0} \epsilon^{*}
$$

and for all $s \geq s^{*}$ :

$$
\left\|\left(\begin{array}{c}
w(s) \\
\partial_{s} w(s)
\end{array}\right)-e^{i \theta_{\infty}}\left(\begin{array}{c}
\kappa\left(\delta_{\infty}, \cdot\right) \\
0
\end{array}\right)\right\|_{\mathcal{H}} \leq C_{0} \epsilon^{*} e^{-\mu_{0}\left(s-s^{*}\right)} .
$$

Remark. Condition (12) is crucial for the conclusion. Indeed, if (13) is satisfied but not (12), we may have a different conclusion, as with the explicit solution 
$w(y, s)=\kappa_{0} \frac{\left(1-\delta^{2}\right)^{\frac{1}{p-1}}}{\left(1+\mu e^{s}+\delta y\right)^{\frac{2}{p-1}}}$ which may be made arbitrarily close to $\kappa(\delta, y)$ and satisfies convergences to 0 as $s \rightarrow+\infty$.

As we said earlier, the third step towards the derivation of the blow-up profile simply uses Proposition 3 and Theorem 4 to get the following:

Theorem 5 (Blow-up profile near a non-characteristic point). If $u$ is a solution of (11) with blow-up curve $\Gamma:\{x \rightarrow T(x)\}$ and $x_{0} \in \mathbb{R}$ is non-characteristic (in the sense as in (3) ), then there exist $\delta_{\infty}\left(x_{0}\right) \in(-1,1), \theta_{\infty}\left(x_{0}\right) \in \mathbb{R}$ and $s^{*}\left(x_{0}\right) \geq$ $-\log T\left(x_{0}\right)$ such that for all $s \geq s^{*}\left(x_{0}\right)$, (14) holds with $\epsilon^{*}=\epsilon_{0}$, where $C_{0}$ and $\epsilon_{0}$ are given in Theorem 4 . Moreover,

$$
\left\|w_{x_{0}}(s)-e^{i \theta_{\infty}\left(x_{0}\right)} \kappa\left(\delta_{\infty}\left(x_{0}\right)\right)\right\|_{H^{1}(-1,1)}+\left\|\partial_{s} w_{x_{0}}(s)\right\|_{L^{2}(-1,1)} \rightarrow 0 \text { as } s \rightarrow \infty .
$$

Remark. From the Sobolev embedding, we know that the convergence takes place also in $L^{\infty}$, in the sense that

$$
\left\|w_{x_{0}}(s)-e^{i \theta_{\infty}\left(x_{0}\right)} \kappa\left(\delta_{\infty}\left(x_{0}\right)\right)\right\|_{L^{\infty}(-1,1)} \rightarrow 0 \text { as } s \rightarrow \infty .
$$

Remark. As we mentioned above one of our difficulties comes from the invariance of the solution under complex rotation, which induces an additional zero-mode in the linearization of equation (5) around $\kappa(\delta, y)$. In order to overcome that difficulty, we use a modulation technique (see Proposition 4.1 below). Let us mention that the extension from the real to complex case has been successfully performed by Filippas and Merle [14] in the case of the semilinear heat equation with Sobolev subcritical non-linearity:

$$
\partial_{t} u=\Delta u+|u|^{p-1} u, \text { with } p>1 \text { and }(N-2) p<N+2 .
$$

We mention that the modulation technique was already crucial in [14] to control the additional zero-mode coming from the invariance of equation (15) under complex rotation. Note however that the adaptation from the real to the complex case for the wave equation is far more difficult, since we have additional problems, coming from the fact that we have to handle non-self-adjoint operators.

This paper is organized as follows: 2

In Section 2, we characterize the set of stationary solutions, proving Proposition

In Section 3, we study the properties of the linearized operator of equation (5) around a non-zero stationary solution.

Finally, Section 4 is devoted to the proof of Proposition 3. Theorem 4 and Theorem 5 .

\section{Characterization of the Stationary SOlutions IN SELF-SIMILAR VARIABLES}

In this section, we prove Proposition 2 which characterizes all $\mathcal{H}_{0}$ solutions of

$$
\frac{1}{\rho}\left(\rho\left(1-y^{2}\right) w^{\prime}\right)^{\prime}-\frac{2(p+1)}{(p-1)^{2}} w+|w|^{p-1} w=0,
$$

the stationary version of (5). As our solution is a complex-valued one, we will use in addition to the techniques of Section 2.3 in 33, other techniques as the determination of the phase and some projections. Note that since 0 and $\kappa_{0} e^{i \theta}$ 
are trivial solutions to equation (5) for any $\theta \in \mathbb{R}$, we see from Lemma C.5 that $\mathcal{T}_{\delta}\left(e^{i \theta} \kappa_{0}\right)=e^{i \theta} \kappa(\delta, y)$ is also a stationary solution to (5). Let us introduce the set

$$
S \equiv\left\{0, e^{i \theta} \kappa(\delta, \cdot),|\delta|<1, \theta \in \mathbb{R}\right\}
$$

and prove that there are no more solutions of (16) in $\mathcal{H}_{0}$ outside the set $S$.

Proof. We first prove $(i i)$, since its proof is short.

(ii) Since we clearly have from the definition (9) that $E(0,0)=0$, we will compute $E\left(e^{i \theta} \kappa(\delta, \cdot), 0\right)$. From (9) and the proof of the real case (page 59 in [33]), we see that

$$
E\left(e^{i \theta} \kappa(\delta, \cdot), 0\right)=E(\kappa(\delta, \cdot), 0)=E\left(\kappa_{0}, 0\right)>0 .
$$

Thus, (11) follows.

(i) Consider $w \in \mathcal{H}_{0}$ a non-zero solution of (16). Let us prove that there are some $\delta \in(-1,1)$ and $\theta \in \mathbb{R}$ such that $w=e^{i \theta} \kappa(\delta, \cdot)$. For this purpose, define

$$
\xi=\frac{1}{2} \log \left(\frac{1+y}{1-y}\right)(\text { that is } y=\tanh \xi) \text { and } v(\xi)=w(y)\left(1-y^{2}\right)^{\frac{1}{p-1}} .
$$

As in the real case, we see from straightforward calculations that $v \not \equiv 0$ is an $H^{1}(\mathbb{R})$ solution to

$$
\partial_{\xi}^{2} v+|v|^{p-1} v-\frac{4}{(p-1)^{2}} v=0, \forall \xi \in \mathbb{R} .
$$

Our aim is to prove the existence of $\theta_{0} \in \mathbb{R}$ and $\xi_{0} \in \mathbb{R}$ such that $v(\xi)=e^{i \theta_{0}} \check{k}\left(\xi+\xi_{0}\right)$ where

$$
\check{k}(\xi)=\frac{\kappa_{0}}{\cosh ^{\frac{2}{p-1}}(\xi)} .
$$

Since $v \in H^{1}(\mathbb{R}) \subset C^{\frac{1}{2}}(\mathbb{R})$, we see that $v$ is a strong $C^{2}$ solution of equation (19). Since $v \not \equiv 0$, there exists $\xi_{0} \in \mathbb{R}$ such that $v\left(\xi_{0}\right) \neq 0$. By invariance of (19) under translation, we may suppose that $\xi_{0}=0$. Let

$$
G^{*}=\{\xi \in \mathbb{R} \mid \check{w}(\xi) \neq 0\},
$$

a non-empty open set by continuity. Note that $G^{*}$ contains some non-empty interval $I$ containing 0 . We also introduce $R(\xi)=|v(\xi)|, \theta$ and $\check{\theta}$ two determinations of the phase given by

$$
\theta(\xi)=\arctan \left(\frac{\operatorname{Im} v(\xi)}{\operatorname{Re} v(\xi)}\right) \text { and } \check{\theta}(\xi)=\operatorname{arccotan}\left(\frac{\operatorname{Re} v(\xi)}{\operatorname{Im} v(\xi)}\right),
$$

and $h: G^{*} \rightarrow \mathbb{R}$ given by

$$
\forall \xi \in G^{*}, h(\xi)=\left\{\begin{array}{l}
\theta^{\prime}(\xi), \text { if } \operatorname{Re} v(\xi) \neq 0 \\
\check{\theta}^{\prime}(\xi), \text { if } \operatorname{Im} v(\xi) \neq 0 .
\end{array}\right.
$$

We claim that $h$ is well defined and that $h \in C^{1}\left(G^{*}\right)$. Indeed, let $\xi_{0} \in \mathbb{R}$ such that $v\left(\xi_{0}\right) \neq 0$. Necessarily, either its real or its imaginary part is non-zero. If for instance $\operatorname{Re} v\left(\xi_{0}\right) \neq 0$, by continuity

$$
\exists \delta_{0}>0, \forall\left|\xi-\xi_{0}\right|<\delta_{0}, \operatorname{Re} \check{w}(\xi) \neq 0,
$$

so $\theta$ is well defined in $\left(\xi_{0}-\delta_{0}, \xi_{0}+\delta_{0}\right)$, and $h$ is well defined and $C^{1}$ in $\left(\xi_{0}-\delta_{0}, \xi_{0}+\delta_{0}\right)$. Now, if $\operatorname{Im} v\left(\xi_{0}\right) \neq 0$, by the same way, we prove that $h$ given by $h(\xi)=\check{\theta}^{\prime}(\xi)$ is well defined and $C^{1}$ in a small interval $\left(\xi_{0}-\delta_{0}, \xi_{0}+\delta_{0}\right)$. 
This definition is non-ambiguous. Indeed, if ever both $\theta$ and $\check{\theta}$ are defined on the same interval $(a, b)$ with $a<b$, then there exists $k \in \mathbb{Z}$ such that

$$
\forall \xi \in(a, b), \theta(\xi)=\check{\theta}(\xi)+2 k \pi .
$$

Differentiating this, we get

$$
\forall \xi \in(a, b), \theta^{\prime}(\xi)=\check{\theta}^{\prime}(\xi) .
$$

Thus, (22) defines $h(\xi)$ with no ambiguity.

Take $\xi \in G^{*}$. Using one of the angle determinations in (21) and projecting equation (19), we see that

$$
\forall \xi \in G^{*},\left\{\begin{array}{l}
R^{\prime \prime}(\xi)-R(\xi) h^{2}(\xi)-c_{0} R(\xi)+R^{p}(\xi)=0, c_{0}=\frac{4}{(p-1)^{2}}, \\
2 R^{\prime}(\xi) h(\xi)+R(\xi) h^{\prime}(\xi)=0 .
\end{array}\right.
$$

Integrating the second equation on the interval $I \subset G^{*}$, we see that for all $\xi \in I$, $h(\xi)=\frac{h(0) R^{2}(0)}{R^{2}(\xi)}$. Plugging this in the first equation, we get

$$
\forall \xi \in I, R^{\prime \prime}(\xi)-\frac{\mu}{R^{3}(\xi)}-c_{0} R(\xi)+R^{p}(\xi)=0, \text { where } \mu=h^{2}(0) R^{4}(0) .
$$

Now let

$$
G=\left\{\xi \in G^{*}, \forall \xi^{\prime} \in I_{\xi}, h\left(\xi^{\prime}\right)=\frac{h(0) R^{2}(0)}{R^{2}\left(\xi^{\prime}\right)}\right\},
$$

where $I_{\xi}=[0, \xi)$ if $\xi \geq 0$ or $I_{\xi}=(\xi, 0]$ if $\xi \leq 0$. Note that $I \subset G$. Now, we give the following:

Lemma 2.1. There exists $\epsilon_{0}>0$ such that

$$
\forall \xi \in G, \forall \xi^{\prime} \in I_{\xi}, 0<\epsilon_{0} \leq\left|v\left(\xi^{\prime}\right)\right| \leq \frac{1}{\epsilon_{0}} .
$$

Proof. Take $\xi \in G$. By definition (25) of $G$, we see that equation (24) is satisfied for all $\xi^{\prime} \in I_{\xi}$. Multiplying $R^{\prime \prime}(\xi)-\frac{\mu}{R^{3}(\xi)}-c_{0} R(\xi)+R^{p}(\xi)=0$ by $R^{\prime}$ and integrating between 0 and $\xi$, we get:

$\forall \xi \in I_{\xi}, \mathcal{E}\left(\xi^{\prime}\right)=\mathcal{E}(0)$, where $\mathcal{E}\left(\xi^{\prime}\right)=\frac{1}{2} R^{\prime 2}\left(\xi^{\prime}\right)+\frac{\mu}{2 R^{2}\left(\xi^{\prime}\right)}-\frac{c_{0}}{2} R^{2}\left(\xi^{\prime}\right)+\frac{R^{p+1}\left(\xi^{\prime}\right)}{p+1}$,

or equivalently,

$$
\forall \xi^{\prime} \in I_{\xi}, F\left(R\left(\xi^{\prime}\right)\right)=\frac{1}{2} R^{\prime}\left(\xi^{\prime}\right)^{2} \geq 0, \text { where } F(r)=-\frac{\mu}{2 r^{2}}+\frac{c_{0}}{2} r^{2}-\frac{r^{p+1}}{p+1}+\mathcal{E}(0) .
$$

Since $F(r) \rightarrow-\infty$ as $r \rightarrow 0$ or $r \rightarrow \infty$, there exists $\epsilon_{0}=\epsilon_{0}(\mu, E(0))>0$ such that $\epsilon_{0} \leq R\left(\xi^{\prime}\right) \leq \frac{1}{\epsilon_{0}}$, which yields the conclusion of Lemma 2.1

We claim the following:

Lemma 2.2. It holds that $G=\mathbb{R}$.

Proof. Note first that by construction, $G$ is a non-empty interval (note that $0 \in I \subset$ $G$ where $I$ is defined right after (20) $)$. We have only to prove that $\sup G=+\infty$, since the fact that $\inf G=-\infty$ can be deduced by replacing $v(\xi)$ by $v(-\xi)$.

By contradiction, suppose that $\sup G=a<+\infty$. By continuity, we have

$$
\forall \xi \in[0, a], h(\xi)=\frac{h(0) R(0)^{2}}{R(\xi)^{2}},
$$


on the one hand. On the other hand, by Lemma 2.1. for all $\xi^{\prime} \in[0, a), 0<\epsilon_{0} \leq$ $\left|v\left(\xi^{\prime}\right)\right| \leq \frac{1}{\epsilon_{0}}$. Using (26) $v(a) \neq 0$, and $a \in G^{*}$. Using (26), we see that $a \in G$. By continuity, we can write for all $\xi \in(a-\delta, a+\delta)$, where $\delta>0$ is small enough,

$$
\left\{\begin{array}{l}
R^{\prime \prime}(\xi)-R(\xi) \theta^{\prime}(\xi)^{2}-c_{0} R(\xi)+R(\xi)^{p}=0, c_{0}=\frac{4}{(p-1)^{2}}, \\
2 R^{\prime}(\xi) h(\xi)+R(\xi) h^{\prime}(\xi)=0 .
\end{array}\right.
$$

From the second equation and (26) applied with $\xi=a$, we see that $h(\xi)=$ $\frac{h(a)(R(a))^{2}}{(R(\xi))^{2}}=\frac{h(0)(R(0))^{2}}{(R(\xi))^{2}}$. Therefore, it follows that $(a, a+\delta) \in E$, which contradicts the fact that $a=\sup G$.

Note from Lemma 2.2 that (23) and (24) hold for all $\xi \in \mathbb{R}$. We claim that $h(0)=0$. Indeed, if not, then by (24), we have $\mu \neq 0$, and since $G=\mathbb{R}$, we see from Lemma 2.1 that for all $\xi \in \mathbb{R},|v(\xi)| \geq \epsilon_{0}$. Therefore $v \notin L^{2}(\mathbb{R})$, which contradicts the fact that $v \in H^{1}(\mathbb{R})$. Thus, $h(0)=0$, and $\mu=0$. By uniqueness of solutions to the second equation of (23), we see that $h(\xi)=0$ for all $\xi \in \mathbb{R}$, so $\theta(\xi)=\theta(0)$ and $v^{\prime}(0)=\lambda e^{i \theta(0)}(\lambda \in \mathbb{R})$. Thus

$$
\left\{\begin{array}{l}
v(0)=R(0) e^{i \theta(0)} \\
v^{\prime}(0)=\lambda e^{i \theta(0)}
\end{array}\right.
$$

Let $W \in H^{1}(\mathbb{R})$ be a real solution of

$$
\left\{\begin{array}{l}
W^{\prime \prime}-c_{0} W+|W|^{p-1} W=0 \\
W(0)=R(0) \\
W^{\prime}(0)=\lambda
\end{array}\right.
$$

By uniqueness of the Cauchy problem of equation (19), we have for all $\xi \in \mathbb{R}, v(\xi)=$ $W(\xi) e^{i \theta(0)}$, and as $v \in H^{1}(\mathbb{R}), W$ is also in $H^{1}(\mathbb{R})$. Since it is well known that the real solutions of (27) in $H^{1}(\mathbb{R})$ are

$$
\text { either } W \equiv 0 \text { or } W(\xi)= \pm \check{k}\left(\xi+\xi_{0}\right) \text { for some } \xi_{0} \in \mathbb{R} \text {, }
$$

for the reader's convenience, we recall the proof in Appendix B. It follows that $v(\xi)=e^{i \theta_{0}} \check{k}\left(\xi+\xi_{0}\right)$ for some $\xi_{0} \in \mathbb{R}$, because $W \not \equiv 0$ and $W>0$. Thus, for $d=\tanh \xi_{0} \in(-1,1)$ and $y=\tanh \xi$, we get

$$
\begin{aligned}
& v(\xi)=e^{i \theta_{0}} \kappa_{0}\left[1-\tanh \left(\xi+\xi_{0}\right)^{2}\right]^{\frac{1}{p-1}}=e^{i \theta_{0}} \kappa_{0}\left[1-\left(\frac{\tanh \xi+\tanh \xi_{0}}{1+\tanh \xi \tanh \xi_{0}}\right)^{2}\right]^{\frac{1}{p-1}} \\
& =e^{i \theta_{0}} \kappa_{0}\left[1-\left(\frac{y+\delta}{1+\delta y}\right)^{2}\right]^{\frac{1}{p-1}}=e^{i \theta_{0}} \kappa_{0}\left[\frac{\left(1-\delta^{2}\right)\left(1-y^{2}\right)^{2}}{(1+\delta y)}\right]^{\frac{1}{p-1}} \\
& =e^{i \theta_{0}} \kappa(\delta, y)\left(1-y^{2}\right)^{\frac{1}{p-1}}
\end{aligned}
$$

By (18), we see that $w(y)=e^{i \theta_{0}} \kappa(\delta, y)$. This concludes the proof of Proposition 2

\section{THE LINEARIZED OPERATOR AROUND A NON-ZERO STATIONARY SOLUTION}

In this section, we study the properties of the linearized operator of equation (5) around the stationary solution $\kappa(\delta, y)$ (10). We recall that in 33 . the authors have treated the real case by introducing $q=\left(q_{1}, q_{2}\right) \in \mathbb{R} \times \mathbb{R}$ and linearizing around $\kappa(\delta, y)$. It turns out that the real part of our linearized operator for complex-valued solution is identical to the real part of the linearized operator in the real case treated 
in 33 . As a matter of fact, we will rely on 33 for the real part and have to invent new methods for the imaginary part.

For any complex number $z$, we use in the following the notation

$$
\check{z}=\operatorname{Re}(z) \text { and } \tilde{z}=\operatorname{Im}(z) \text {. }
$$

If we introduce $q=\left(q_{1}, q_{2}\right)=\left(\begin{array}{l}q_{1} \\ q_{2}\end{array}\right) \in \mathbb{C} \times \mathbb{C}$ for all $s \in\left[s_{0}, \infty\right)$, for a given $s_{0} \in \mathbb{R}$, by

$$
\left(\begin{array}{c}
w(y, s) \\
\partial_{s} w(y, s)
\end{array}\right)=\left(\begin{array}{c}
\kappa(\delta, y) \\
0
\end{array}\right)+\left(\begin{array}{c}
q_{1}(y, s) \\
q_{2}(y, s)
\end{array}\right)
$$

then we see from equation (5) that $q$ satisfies the following equation for all $s \geq s_{0}$ :

$$
\frac{\partial}{\partial s}\left(\begin{array}{l}
q_{1} \\
q_{2}
\end{array}\right)=L_{\delta}\left(\begin{array}{l}
q_{1} \\
q_{2}
\end{array}\right)+\left(\begin{array}{c}
0 \\
f_{\delta}\left(q_{1}\right)
\end{array}\right)
$$

where

$$
\begin{gathered}
L_{\delta}\left(\begin{array}{c}
q_{1} \\
q_{2}
\end{array}\right)=\left(\begin{array}{c}
q_{2} \\
\mathcal{L} q_{1}+\check{\psi}(\delta, y) \check{q}_{1}+i \tilde{\psi}(\delta, y) \tilde{q_{1}}-\frac{p+3}{p-1} q_{2}-2 y \partial_{y} q_{2}
\end{array}\right), \\
\check{\psi}(\delta, y)=p \kappa(\delta, y)^{p-1}-\frac{2(p+1)}{(p-1)^{2}}, \\
\tilde{\psi}(\delta, y)=\kappa(\delta, y)^{p-1}-\frac{2(p+1)}{(p-1)^{2}}, \\
f_{\delta}\left(q_{1}\right)=\check{f}_{\delta}\left(\check{q}_{1}, \tilde{q}_{1}\right)+i \tilde{f}_{\delta}\left(\check{q}_{1}, \tilde{q}_{1}\right),
\end{gathered}
$$

where $\check{f}_{\delta}\left(\check{q}_{1}, \tilde{q}_{1}\right)=\left|\kappa(\delta, y)+q_{1}\right|^{p-1}\left(\kappa(\delta, y)+\check{q}_{1}\right)-\kappa(\delta, y)^{p}-p \kappa^{p-1}(\delta, y) \check{q}_{1}$,

$$
\tilde{f}_{\delta}\left(\check{q}_{1}, \tilde{q}_{1}\right)=\left|\kappa(\delta, y)+q_{1}\right|^{p-1} \tilde{q}_{1}-\kappa^{p-1}(\delta, y) \tilde{q}_{1} .
$$

From (29), dissociating the real and imaginary parts, we get for all $s \geq s_{0}$ :

$$
\frac{\partial}{\partial s}\left(\begin{array}{c}
\check{q}_{1} \\
\check{q}_{2}
\end{array}\right)=\check{L}_{\delta}\left(\begin{array}{c}
\check{q}_{1} \\
\check{q}_{2}
\end{array}\right)+\left(\begin{array}{c}
0 \\
\check{f}_{\delta}
\end{array}\right)
$$

where

$$
\check{L}_{\delta}\left(\begin{array}{c}
\check{q}_{1} \\
\check{q}_{2}
\end{array}\right)=\left(\begin{array}{c}
\check{q}_{2} \\
\mathcal{L} \check{q}_{1}+\check{\psi}(\delta, y) \check{q}_{1}-\frac{p+3}{p-1} \check{q}_{2}-2 y \partial_{y} \check{q}_{2}
\end{array}\right),
$$

and

$$
\frac{\partial}{\partial s}\left(\begin{array}{c}
\tilde{q}_{1} \\
\tilde{q}_{2}
\end{array}\right)=\tilde{L}_{\delta}\left(\begin{array}{c}
\tilde{q}_{1} \\
\tilde{q}_{2}
\end{array}\right)+\left(\begin{array}{c}
0 \\
\tilde{f}_{\delta}
\end{array}\right)
$$

where

$$
\tilde{L}_{\delta}\left(\begin{array}{c}
\tilde{q}_{1} \\
\tilde{q}_{2}
\end{array}\right)=\left(\begin{array}{c}
\tilde{q}_{2} \\
\mathcal{L} \tilde{q}_{1}+\tilde{\psi}(\delta, y) \tilde{q}_{1}-\frac{p+3}{p-1} \tilde{q}_{2}-2 y \partial_{y} \tilde{q}_{2}
\end{array}\right) .
$$

Remark. From (29) we see that for $q=\left(\begin{array}{c}\check{q_{1}} \\ \check{q_{2}}\end{array}\right)+i\left(\begin{array}{c}\tilde{q_{1}} \\ \tilde{q_{2}}\end{array}\right)$, we have

$$
L_{\delta}\left(\begin{array}{c}
q_{1} \\
q_{2}
\end{array}\right)=\check{L}_{\delta}\left(\begin{array}{c}
\check{q_{1}} \\
\check{q_{2}}
\end{array}\right)+i \tilde{L}_{\delta}\left(\begin{array}{c}
\tilde{q_{1}} \\
\tilde{q_{2}}
\end{array}\right) \text {. }
$$

Note that the operator $\check{L}_{\delta}(32)$ already appears in the real case studied in [33]. For that reason, we recall from that paper the properties of $\check{L}_{\delta}$, and focus here on the 
properties of $\tilde{L}_{\delta}$, which is one of the novelties of our work. Note from (7) that we have

$$
\|q\|_{\mathcal{H}}=[\phi(q, q)]^{\frac{1}{2}}<+\infty,
$$

where the hermitian inner product $\phi$ is defined by

$$
\phi(q, r)=\phi\left(\left(\begin{array}{l}
q_{1} \\
q_{2}
\end{array}\right),\left(\begin{array}{l}
r_{1} \\
r_{2}
\end{array}\right)\right)=\int_{-1}^{1}\left(q_{1} \bar{r}_{1}+q_{1}^{\prime} \bar{r}_{1}^{\prime}\left(1-y^{2}\right)+q_{2} \bar{r}_{2}\right) \rho(y) d y .
$$

Using integration by parts and the definition of $\mathcal{L}(\underline{6})$, we have the following:

$$
\phi(q, r)=\int_{-1}^{1}\left(q_{1}\left(-\mathcal{L} \bar{r}_{1}+\bar{r}_{1}\right)+q_{2} \bar{r}_{2}\right) \rho(y) d y .
$$

We note that $q \in \mathcal{H}$ if and only if $\check{q} \in \mathcal{H}$ and $\tilde{q} \in \mathcal{H}$, and

$$
\|q\|_{\mathcal{H}}^{2}=\|\check{q}\|_{\mathcal{H}}^{2}+\|\tilde{q}\|_{\mathcal{H}}^{2} \text {. }
$$

This section is organized as follows:

-We first recall some spectral properties of $\check{L}_{\delta}$ which was proved by Merle and Zaag in 33 .

-Then, we focus on the study of $\tilde{L}_{\delta}$, precisely, we compute $\tilde{L}_{\delta}^{*}$ the conjugate operator of $\tilde{L}_{\delta}$ and we give a zero direction for it.

-Using the projection on the eigenspace of $\tilde{L}_{\delta}$, we introduce a function which will capture the dispersive character of equation (29), and give some dispersive estimates in order to prove Theorem 4 .

3.1. Spectral properties of $\check{L}_{\delta}$. From Section 4 in [33], we know that $\check{L}_{\delta}$ has two non-negative eigenvalues $\lambda=1$ and $\lambda=0$ with eigenfunctions

$$
\check{F}_{1}(\delta, y)=\left(1-\delta^{2}\right)^{\frac{p}{p-1}}\left(\begin{array}{c}
(1+\delta y)^{-\frac{p+1}{p-1}} \\
(1+\delta y)^{-\frac{p+1}{p-1}}
\end{array}\right) \text { and } \check{F}_{0}(\delta, y)=\left(1-\delta^{2}\right)^{\frac{1}{p-1}}\left(\begin{array}{c}
\frac{y+\delta}{(1+\delta y)^{\frac{p+1}{p-1}}} \\
0
\end{array}\right) \text {. }
$$

Note that for some $C_{0}>0$ and any $\lambda \in\{0,1\}$, we have

$$
\forall|\delta|<1, \quad \frac{1}{C_{0}} \leq\left\|\check{F}_{\lambda}^{\delta}\right\|_{\mathcal{H}} \leq C_{0} \text { and }\left\|\partial_{\delta} \check{F}_{\lambda}^{\delta}\right\|_{\mathcal{H}} \leq \frac{C_{0}}{1-\delta^{2}}
$$

We also know that $\check{L}_{\delta}^{*}$ the conjugate operator of $\check{L}_{\delta}$ with respect to $\phi$ is given by

$$
\check{L}_{\delta}^{*}\left(\begin{array}{c}
r_{1} \\
r_{2}
\end{array}\right)=\left(\begin{array}{c}
\check{R}_{\delta}\left(r_{2}\right) \\
-\mathcal{L} r_{1}+r_{1}+\frac{p+3}{p-1} r_{2}+2 y r_{2}^{\prime}-\frac{8}{(p-1)} \frac{r_{2}}{\left(1-y^{2}\right)}
\end{array}\right)
$$

for any $\left(r_{1}, r_{2}\right) \in(\mathcal{D}(\mathcal{L}))^{2}$, where $r=\check{R}_{\delta}\left(r_{2}\right)$ is the unique solution of

$$
-\mathcal{L} r+r=\mathcal{L} r_{2}+\check{\psi}(\delta, y) r_{2} .
$$

Here, the domain $\mathcal{D}(\mathcal{L})$ of $\mathcal{L}$ defined in (6) is the set of all $r \in L_{\rho}^{2}$ such that $\mathcal{L} r \in L_{\rho}^{2}$. Furthermore, $\check{L}_{\delta}^{*}$ has two non-negative eigenvalues $\lambda=0$ and $\lambda=1$ with eigenfunctions $\check{W}_{\lambda}$ such that

$$
\check{W}_{1,2}(\delta, y)=\check{c}_{1} \frac{\left(1-y^{2}\right)(1-\delta)^{\frac{1}{p-1}}}{(1+\delta y)^{\frac{p+1}{p-1}}}, \check{W}_{0,2}(\delta, y)=\check{c}_{0} \frac{(y+\delta)(1-\delta)^{\frac{1}{p-1}}}{(1+\delta y)^{\frac{p+1}{p-1}}},
$$


with 1

$$
\frac{1}{\check{c}_{\lambda}}=2\left(\frac{2}{p-1}+\lambda\right) \int_{-1}^{1}\left(\frac{y^{2}}{1-y^{2}}\right)^{1-\lambda} \rho(y) d y,
$$

and $\check{W}_{\lambda, 1}(\delta, \cdot)$ is the unique solution of the equation

$$
-\mathcal{L} r+r=\left(\lambda-\frac{p+3}{p-1}\right) r_{2}-2 y r_{2}^{\prime}+\frac{8}{p-1} \frac{r_{2}}{1-y^{2}}
$$

with $r_{2}=\check{W}_{\lambda, 2}(\delta, \cdot)$. Note that we have the following relations for $\lambda=0$ or $\lambda=1$ :

$$
\phi\left(\check{W}_{\lambda}(\delta, \cdot), \check{F}_{\lambda}(\delta, \cdot)\right)=1 \text { and } \phi\left(\check{W}_{\lambda}(\delta, \cdot), \check{F}_{1-\lambda}(\delta, \cdot)\right)=0 .
$$

Let us introduce for $\lambda \in\{0,1\}$ the projectors $\check{\pi}_{\lambda}^{\delta}(r)$, and $\check{\pi}_{-}^{\delta}(r)$ for any $r \in \mathcal{H}$ by

$$
\begin{gathered}
\check{\pi}_{\lambda}^{\delta}(r)=\phi\left(\check{W}_{\lambda}(\delta, \cdot), r\right), \\
r=\check{\pi}_{0}^{\delta}(r) \check{F}_{0}(\delta, \cdot)+\check{\pi}_{1}^{\delta}(r) \check{F}_{1}(\delta, \cdot)+\check{\pi}_{-}^{\delta}(r),
\end{gathered}
$$

and the space

$$
\check{\mathcal{H}}_{-}^{\delta} \equiv\left\{r \in \mathcal{H} \mid \check{\pi}_{1}^{\delta}(r)=\check{\pi}_{0}^{\delta}(r)=0\right\} .
$$

Introducing the bilinear form

$$
\check{\varphi}_{\delta}(q, r)=\int_{-1}^{1}\left(-\check{\psi}(\delta, \cdot) q_{1} r_{1}+q_{1}^{\prime} r_{1}^{\prime}\left(1-y^{2}\right)+q_{2} r_{2}\right) \rho d y
$$

where $\check{\psi}(\delta, y)$ is defined in (30), we recall from Proposition 4.7 on page 90 in 33 . that there exists $C_{0}>0$ such that for all $|\delta|<1$, for all $r \in \tilde{\mathcal{H}}_{-}^{\delta}$,

$$
\frac{1}{C_{0}}\|r\|_{\mathcal{H}}^{2} \leq \check{\varphi}_{\delta}(r, r) \leq C_{0}\|r\|_{\mathcal{H}}^{2} \text {. }
$$

In the following sections, we follow the method of 33] to study the spectral properties of $\tilde{L}_{\delta}$.

3.2. A zero direction of $\tilde{L}_{\delta}$. Let us show that $\lambda=0$ is an eigenvalue for $\tilde{L}_{\delta}$. We claim the following:

Lemma 3.1 (Zero direction of $\tilde{L}_{\delta}$ ).

(i) For all $|\delta|<1, \lambda=0$ is an eigenvalue of the linear operator $\tilde{L}_{\delta}$ and its corresponding eigenfunction is

$$
\tilde{F}_{0}(\delta, y)=\left(\begin{array}{c}
\kappa(\delta, y) \\
0
\end{array}\right)
$$

(ii) Moreover, it holds for some $C_{0}>0$ that

$$
\forall|\delta|<1, \quad \frac{1}{C_{0}} \leq\left\|\tilde{F}_{0}(\delta, \cdot)\right\|_{\mathcal{H}} \leq C_{0} \text { and }\left\|\partial_{\delta} \tilde{F}_{0}(\delta, \cdot)\right\|_{\mathcal{H}} \leq \frac{C_{0}}{1-\delta^{2}} .
$$

Remark. There is a more geometrical way to see that $\lambda=0$ is an eigenvalue for $\tilde{L}_{\delta}$ and $\check{L}_{\delta}$ (in other words, a double eigenvalue for $L_{\delta}$ given in (30) $)$ : simply note that equation (5) has a two-parameter family of stationary solutions

$$
K(\delta, \theta, y)=\left(e^{i \theta} \kappa(\delta, y), 0\right)
$$

\footnotetext{
${ }^{1}$ In section 4 of 33 , we had non-explicit normalizing constants $\check{c}_{\lambda}=\check{c}_{\lambda}(\delta)$. In Lemma 2.4 in [39], the authors compute the explicit dependence of $\check{c}_{\lambda}(\delta)$.
} 
hence, $\partial_{\delta} K(\delta, 0, y)=\left(\partial_{\delta} \kappa(\delta, y), 0\right)$ and $\partial_{\theta} K(\delta, 0, y)=(i \kappa(\delta, y), 0)$ are eigenfunctions of the linearized operator of equation (15) around $K(\delta, 0, y)=(\kappa(\delta, y), 0)$, which is precisely the operator $L_{\delta}$. Splitting $L_{\delta}$ into real and imaginary parts shows that $\left(\partial_{\delta} \kappa(\delta, y), 0\right)$ and $(\kappa(\delta, y), 0)$ are eigenfunctions of $\check{L}_{\delta}$ and $\tilde{L}_{\delta}$, respectively. A simple calculation shows indeed that $\left(\partial_{\delta} \kappa(\delta, y), 0\right)$ is proportional to $\tilde{F}_{0}(\delta, y)$ given in (36). The fact that $\lambda=1$ is an eigenvalue of $L_{\delta}$ follows from similar ideas: noting that

$$
\bar{K}(\delta, \mu, y, s)=\left(\frac{\kappa_{0}\left(1-\delta^{2}\right)^{\frac{1}{p-1}}}{\left(1+\mu e^{s}+\delta y\right)^{\frac{2}{p-1}}},-\frac{2 \mu e^{s}}{p-1} \frac{\kappa_{0}\left(1-\delta^{2}\right)^{\frac{1}{p-1}}}{\left(1+\mu e^{s}+\delta y\right)^{\frac{p+1}{p-1}}}\right)
$$

is an explicit solution of equation (5) with $\bar{K}(\delta, 0, y, s)=(\kappa(\delta, y), 0)$, when $\mu=0$, differentiating with respect to the new parameter $\mu$, we obtain an eigenfunction for $L_{\delta}$ with $\lambda=1$.

Proof. $(i)$ As $\kappa(\delta, y)$ is a stationary solution of (5), it satisfies (16), hence

$$
\mathcal{L} \kappa(\delta, y)-\frac{2(p+1)}{(p-1)^{2}} \kappa(\delta, y)+\kappa(\delta, y)^{p}=0 .
$$

By definition (34) of $\tilde{L}_{\delta}$, we see that $\tilde{L}_{\delta}\left(\begin{array}{c}\kappa(\delta, y) \\ 0\end{array}\right)=0$.

(ii) Noting that $\kappa(\delta, y)=\mathcal{T}_{\delta}\left(\kappa_{0}\right)$ where the transformation $\mathcal{T}_{\delta}$ is defined in (176), applying Lemma C.6 and using (10), we get the first bound. In order to prove the second one, we recall the following integral calculation rules from [33]:

Claim 3.2. Consider for some $\alpha>-1$ and $\beta \in \mathbb{R}$ the following integral:

$$
I(\delta)=\int_{-1}^{1} \frac{\left(1-y^{2}\right)^{\alpha}}{(1+\delta y)^{\beta}} d y .
$$

(i) if $\alpha+1-\beta>0$, then $\frac{1}{K} \leq I(\delta) \leq K$;

(ii) if $\alpha+1-\beta=0$, then $\frac{1}{K} \leq \frac{I(\delta)}{\left|\log \left(1-\delta^{2}\right)\right|} \leq K$;

(iii) if $\alpha+1-\beta<0$, then $\frac{1}{K} \leq I(\delta)\left(1-\delta^{2}\right)^{-(\alpha+1)+\beta} \leq K$.

Proof. See page 84 of 33 .

Using the definition of $\tilde{F}_{0}$ (42), the fact that

$$
\forall(\delta, y) \in(-1,1)^{2},|y+\delta|+\left|1-\delta^{2}\right|+\left(1-y^{2}\right) \leq C(1+\delta y)
$$

and straightforward computations we see that

$$
\left|\partial_{\delta} \tilde{F}_{0}(\delta, y)\right| \leq C \frac{\left(1-\delta^{2}\right)^{\frac{2-p}{p-1}}}{(1+\delta y)^{\frac{2}{p-1}}}, \quad\left|\partial_{\delta, y}^{2} \tilde{F}_{0}(\delta, y)\right| \leq C \frac{\left(1-\delta^{2}\right)^{\frac{2-p}{p-1}}}{(1+\delta y)^{\frac{p+1}{p-1}}} .
$$

Using this and Claim 3.2, we see that (43) holds for $\tilde{F}_{0}$.

3.3. The conjugate operator $\tilde{L}_{\delta}^{*}$. In this step of the work of [33], the authors have computed $\check{L}_{\delta}^{*}$ by simple calculations using the definition of the conjugate, namely that $\phi\left(\check{L}_{\delta}(q), r\right)=\phi\left(q, \check{L}_{\delta}^{*}(r)\right)$ and the fact that $\mathcal{L}$ is self-adjoint. In the same way, we introduce, in the following, the conjugate operator of $\tilde{L}_{\delta}$ with respect to $\phi$. 
Lemma 3.3 (The conjugate operator of $\tilde{L}_{\delta}$ with respect to $\phi$ ). For all $|\delta|<1$, the operator $\tilde{L}_{\delta}^{*}$ conjugate of $\tilde{L}_{\delta}$ with respect to $\phi$ is given by

$$
\tilde{L}_{\delta}^{*}\left(\begin{array}{c}
r_{1} \\
r_{2}
\end{array}\right)=\left(\begin{array}{c}
\tilde{R}_{\delta}\left(r_{2}\right) \\
-\mathcal{L} r_{1}+r_{1}+\frac{p+3}{p-1} r_{2}+2 y r_{2}^{\prime}-\frac{8}{(p-1)} \frac{r_{2}}{\left(1-y^{2}\right)}
\end{array}\right)
$$

for any $\left(r_{1}, r_{2}\right) \in(\mathcal{D}(\mathcal{L}))^{2}$, where $g=\tilde{R}_{\delta}\left(r_{2}\right)$ is the unique solution of

$$
-\mathcal{L} g+g=\mathcal{L} r_{2}+\tilde{\psi}(\delta, y) r_{2} .
$$

Proof. The proof is the same as the proof of Lemma 4.1 on page 81 in 33 .

In the following, we give an eigenfunction of $\tilde{L}_{\delta}^{*}$ associated to the eigenvalue $\lambda=0$.

Lemma 3.4 (Eigenfunction of $\tilde{L}_{\delta}^{*}$ for the eigenvalue $\lambda=0$ ).

(i) (Existence) For all $|\delta|<1$, there exists $\tilde{W}_{0} \in \mathcal{H}$ continuous in terms of $d$ such that $\tilde{L}_{\delta}^{*}\left(\tilde{W}_{0}\right)=0$ where

$$
\tilde{W}_{0,2}(\delta, y)=\tilde{c}_{0} \kappa(\delta, y) \text { and } \frac{1}{\tilde{c}_{0}}=\frac{4 \kappa_{0}^{2}}{p-1} \int_{-1}^{1} \frac{\rho(y)}{1-y^{2}} d y
$$

and $\tilde{W}_{0,1}$ is the unique solution of the equation

$$
-\mathcal{L} g+g=-\frac{p+3}{p-1} r_{2}-2 y r_{2}^{\prime}+\frac{8}{p-1} \frac{r_{2}}{1-y^{2}}
$$

with $r_{2}=\tilde{W}_{0,2}$. Moreover, we have

$$
\phi\left(\tilde{W}_{0}, \tilde{F}_{0}\right)=1 .
$$

(ii) (Normalization) There exists $C_{0}>0$ such that for $|\delta|<1$,

$$
\left\|\tilde{W}_{0}(\delta, \cdot)\right\|_{\mathcal{H}} \leq C_{0} \quad \text { and }\left\|\partial_{\delta} \tilde{W}_{0}(\delta, \cdot)\right\|_{\mathcal{H}} \leq \frac{C_{0}}{1-\delta^{2}} .
$$

Before proving this lemma, let us recall the result from 33 .

Claim 3.5. For any $r_{2} \in \mathcal{H}_{0}$, the equation (48) has a unique solution $g \in \mathcal{H}_{0}$ (8) such that

$$
\|g\|_{\mathcal{H}_{0}} \leq C\left\|r_{2}\right\|_{\mathcal{H}_{0}} .
$$

Proof. See Claim 4.5 on page 86 in 33 .

Proof of Lemma 3.4. ( $i$ ) From the definition of $\tilde{L}_{\delta}^{*}$ (see (45)), $\tilde{W}_{0}(\delta, \cdot)=$ $\left(\tilde{W}_{0,1}(\delta, \cdot), \tilde{W}_{0,2}(\delta, \cdot)\right)$ is an eigenfunction for the eigenvalue $\lambda=0$ if and only if

(51) $\int \tilde{R}_{\delta}\left(\tilde{W}_{0,2}(\delta, \cdot)\right)=0$,

$(52)\left\{-\mathcal{L} \tilde{W}_{0,1}(\delta, \cdot)+\tilde{W}_{0,1}(\delta, \cdot)+\frac{p+3}{p-1} \tilde{W}_{0,2}(\delta, \cdot)+2 y \partial_{y} \tilde{W}_{0,2}(\delta, \cdot)-\frac{8}{(p-1)} \frac{\tilde{W}_{0,2}(\delta, \cdot)}{\left(1-y^{2}\right)}=0\right.$.

Note that $\tilde{R}_{\delta}\left(r_{2}\right)$ is the unique solution of (46). Therefore, if $\tilde{W}_{0}$ is a solution of (51)-(52), then we have

$$
\mathcal{L} \tilde{W}_{0,2}(\delta, \cdot)+\tilde{\psi}(\delta, \cdot) \tilde{W}_{0,2}(\delta, \cdot)=0 .
$$

Note also that, since $\kappa(\delta, y)$ is a stationary solution of equation (5), it follows that

$$
\mathcal{L} \kappa(\delta, y)+\tilde{\psi} \kappa(\delta, y)=0 .
$$


This suggests that we take $\tilde{W}_{0,2}(\delta, y)=\tilde{c}_{0}(\delta) \kappa(\delta, y)$ with $\tilde{c}_{0}(\delta) \neq 0$ and $\tilde{W}_{0,1}$ the unique solution of (52) (note that $\kappa(\delta, \cdot) \in \mathcal{H}_{0}$ by definition (10) and use Claim 3.5 for the existence and uniqueness of $\left.\tilde{W}_{0,1}(\delta, \cdot)\right)$. In this step, we will try to normalize $\tilde{W}_{0}$. From the definition of $\phi(35)$, Lemma 3.1 and (48), we write

$$
\begin{aligned}
\phi\left(\tilde{W}_{0}(\delta, \cdot), \tilde{F}_{0}(\delta, \cdot)\right)=\int_{-1}^{1}\left(\left(-\mathcal{L} \tilde{W}_{0,1}(\delta, y)\right.\right. & \left.+\tilde{W}_{0,1}(\delta, y)\right) \kappa(\delta, y) \\
& \left.+\tilde{W}_{0,1}(\delta, y) \kappa(\delta, y)\right) \rho(y) d y \\
=\int_{-1}^{1}\left(-\frac{p+3}{p-1} \tilde{W}_{0,2}(\delta, y)\right. & -2 y \tilde{W}_{0,2}^{\prime}(\delta, y) \\
& \left.+\frac{8}{p-1} \frac{\tilde{W}_{0,2}(\delta, y)}{\left(1-y^{2}\right)}\right) \kappa(\delta, y) \rho(y) d y .
\end{aligned}
$$

Note in particular that $\tilde{W}_{0,2}(\delta, y)=\tilde{c}_{0}(\delta) \kappa(\delta, y)$, so

$$
\begin{aligned}
& \phi\left(\tilde{W}_{0}(\delta, \cdot), \tilde{F}_{0}(\delta, \cdot)\right) \\
& \quad=\tilde{c}_{0}(\delta)\left[\int_{-1}^{1}\left(-\frac{p+3}{p-1}+\frac{8}{(p-1)\left(1-y^{2}\right)}\right) \kappa^{2}(\delta, y) \rho(y) d y+\int_{-1}^{1} \kappa^{2}(\delta, y)(y \rho(y))^{\prime} d y\right] \\
& \quad=\tilde{c}_{0}(\delta) \int_{-1}^{1}\left(-\frac{p+3}{p-1}+\frac{8}{(p-1)\left(1-y^{2}\right)}+1-\frac{4 y^{2}}{(p-1)\left(1-y^{2}\right)}\right) \kappa^{2}(\delta, y) \rho(y) d y \\
& =\tilde{c}_{0}(\delta) \frac{4}{p-1} \kappa_{0}^{2}\left(1-\delta^{2}\right)^{\frac{2}{p-1}} \int_{-1}^{1} \frac{1}{(1+\delta y)^{\frac{4}{p-1}}} \frac{\rho(y)}{1-y^{2}} d y .
\end{aligned}
$$

Performing the change of variable $Y=\frac{y+\delta}{1+\delta y}$, we get

$$
\phi\left(\tilde{W}_{0}(\delta, \cdot), \tilde{F}_{0}(\delta, \cdot)\right)=\tilde{c}_{0}(\delta) \frac{4}{p-1} \kappa_{0}^{2} \int_{-1}^{1}\left(1-Y^{2}\right)^{\frac{3-p}{p-1}} d Y .
$$

Therefore, in order to get $\phi\left(\tilde{W}_{0}, \tilde{F}_{0}\right)=1$, it is enough to fix $\tilde{c}_{0}(\delta)$ as a positive constant independent from $\delta$ as stated in (47).

(ii) (Normalization) Since $\tilde{W}_{0,1}$ and $\partial_{\delta} \tilde{W}_{0,1}$ are solutions to equation (48), respectively with $r_{2}=\tilde{W}_{0,2}$ and $r_{2}=\partial_{\delta} \tilde{W}_{0,2}$, we see from Claim 3.5 that for all $|\delta|<1$,

$$
\left\|\tilde{W}_{0}\right\|_{\mathcal{H}} \leq C_{0}\left\|\tilde{W}_{0,2}\right\|_{\mathcal{H}_{0}} \text { and }\left\|\partial_{\delta} \tilde{W}_{0}\right\|_{\mathcal{H}} \leq C_{0}\left\|\partial_{\delta} \tilde{W}_{0,2}\right\|_{\mathcal{H}_{0}} .
$$

Using (44) together with the definition of $\tilde{W}_{0,2}$ and straightforward computations, we see that for all $|\delta|<1$ and $|y|<1$,

$$
\begin{gathered}
\left|\tilde{W}_{0,2}(\delta, y)\right| \leq C \frac{\left(1-\delta^{2}\right)^{\frac{1}{p-1}}}{(1+\delta y)^{\frac{2}{p-1}}}, \quad\left|\partial_{y} \tilde{W}_{0,2}(\delta, y)\right| \leq C \frac{\left(1-\delta^{2}\right)^{\frac{1}{p-1}}}{(1+\delta y)^{\frac{p+1}{p-1}}} \\
\left|\partial_{\delta} \tilde{W}_{0,2}(\delta, y)\right| \leq C \frac{\left(1-\delta^{2}\right)^{\frac{2-p}{p-1}}}{(1+\delta y)^{\frac{2}{p-1}}}, \quad\left|\partial_{\delta, y}^{2} \tilde{W}_{0,2}(\delta, y)\right| \leq C \frac{\left(1-\delta^{2}\right)^{\frac{2-p}{p-1}}}{(1+\delta y)^{\frac{p+1}{p-1}}} .
\end{gathered}
$$

Since we have by this, by Claim 3.2 and by the definition of the norm in $\mathcal{H}_{0}$, $\left\|\tilde{W}_{0,2}\right\|_{\mathcal{H}_{0}}+\left(1-\delta^{2}\right)\left\|\partial_{\delta} \tilde{W}_{0,2}\right\|_{\mathcal{H}_{0}} \leq C_{0}$, we see that (50) follows by (54). This concludes the proof of Lemma 3.4 . 
3.4. Expansion of $q$ with respect to the eigenspaces of $\tilde{L}_{\delta}$. In the following, we expand any $r \in \mathcal{H}$ with respect to the eigenspaces of $\tilde{L}_{\delta}$ partially computed in Lemma 3.1. We claim the following:

Definition 3.6 (Expansion of $r$ with respect to the eigenspaces of $\tilde{L}_{\delta}$ ). Consider $r \in \mathcal{H}$ and introduce

$$
\tilde{\pi}_{0}^{\delta}(r)=\phi\left(\tilde{W}_{0}(\delta, \cdot), r\right),
$$

where $\tilde{W}_{0}(\delta, \cdot)$ is the eigenfunction of $\tilde{L}_{\delta}^{*}$ computed in Lemma 3.4. and $\tilde{\pi}_{-}^{\delta}(r)$ is defined by

$$
r=\tilde{\pi}_{0}^{\delta}(r) \tilde{F}_{0}(\delta, \cdot)+\tilde{\pi}_{-}^{\delta}(r) .
$$

Applying the operator $\tilde{\pi}_{0}^{\delta}$ to $(\underline{56})$, we write

$$
\tilde{\pi}_{0}^{\delta}(r)=\tilde{\pi}_{0}^{\delta}(r) \tilde{\pi}_{0}^{\delta}\left(\tilde{F}_{0}(\delta, \cdot)\right)+\tilde{\pi}_{0}^{\delta}\left(\tilde{\pi}_{-}^{\delta}(r)\right) .
$$

By (49),

$$
\tilde{\pi}_{0}^{\delta}\left(\tilde{F}_{0}(\delta, \cdot)\right)=\phi\left(\tilde{W}_{0}(\delta, \cdot), \tilde{F}_{0}(\delta, \cdot)\right)=1
$$

therefore,

$$
\phi\left(\tilde{W}_{0}(\delta, \cdot), \tilde{\pi}_{-}^{\delta}(r)\right)=\tilde{\pi}_{0}^{\delta}\left(\tilde{\pi}_{-}^{\delta}(r)\right)=0
$$

Thus, we have

$$
\tilde{\pi}_{-}^{\delta}(r) \in \tilde{\mathcal{H}}_{-}^{\delta} \equiv\left\{r \in \mathcal{H} \mid \tilde{\pi}_{0}^{\delta}(r)=0\right\} .
$$

Remark. Note that if $r \in \tilde{\mathcal{H}}_{-}^{\delta}$, then $\tilde{\pi}_{-}^{\delta}(r)=r$ (just use (56) and (57)) and $\tilde{L}_{\delta} r \in$ $\tilde{\mathcal{H}}_{-}^{\delta}$. Indeed, using the definition of $\pi_{0}^{\delta}$ (55), the definition of $\tilde{L}_{\delta}^{*}$ and Lemma 3.4, we write $\tilde{\pi}_{0}^{\delta}\left(\tilde{L}_{\delta} r\right)=\phi\left(\tilde{W}_{0}(\delta, \cdot), \tilde{L}_{\delta} r\right)=\phi\left(\tilde{L}_{\delta}^{*} \tilde{W}_{0}(\delta, \cdot), r\right)=0$. Moreover $\tilde{\pi}_{-}^{\delta}\left(\tilde{F}_{0}(\delta, \cdot)\right)=0$ (just use (56) with $r=\tilde{F}_{0}(\delta, \cdot)$ and (49)).

Remark. Note that $\tilde{\pi}_{0}^{\delta}(r)$ is the projection of $r$ on the eigenfunction of $\tilde{L}_{\delta}$ associated to $\lambda=0$, and $\tilde{\pi}_{-}^{\delta}(r)$ is the negative part of $r$.

3.5. Equivalent norms on $\mathcal{H}$ and $\tilde{\mathcal{H}}_{-}^{\delta}$ adapted to the dispersive structure. We introduce

$$
\begin{aligned}
\tilde{\varphi}_{\delta}(q, r) & =\int_{-1}^{1}\left(-\tilde{\psi}(\delta, \cdot) q_{1} r_{1}+q_{1}^{\prime} r_{1}^{\prime}\left(1-y^{2}\right)+q_{2} r_{2}\right) \rho d y \\
& =\int_{-1}^{1}\left(-q_{1}\left(\mathcal{L} r_{1}+\tilde{\psi}(\delta, \cdot) r_{1}\right)+q_{2} r_{2}\right) \rho d y .
\end{aligned}
$$

Proposition 3.7 (Equivalence in $\tilde{\mathcal{H}}_{-}^{\delta}$ of the $\mathcal{H}$ norm and the $\tilde{\varphi}_{\delta}$ norm). There exists $C_{0}>0$ such that for all $|\delta|<1$, the following hold:

(i) (Equivalence of norms in $\tilde{\mathcal{H}}_{-}^{\delta}$ ) For all $r \in \tilde{\mathcal{H}}_{-}^{\delta}$,

$$
\frac{1}{C_{0}}\|r\|_{\mathcal{H}}^{2} \leq \tilde{\varphi}_{\delta}(r, r) \leq C_{0}\|r\|_{\mathcal{H}}^{2} .
$$

(ii) (Equivalence of norms in $\mathcal{H}$ ) For all $r \in \mathcal{H}$,

$$
\frac{1}{C_{0}}\|r\|_{\mathcal{H}} \leq\left(\left|\tilde{\pi}_{0}^{\delta}(r)\right|+\sqrt{\tilde{\varphi}_{\delta}\left(r_{-}, r_{-}\right)}\right) \leq C_{0}\|r\|_{\mathcal{H}} \text {, where } r_{-}=\tilde{\pi}_{-}^{\delta}(r) .
$$


We introduce for all $\epsilon>0$

(59)

$$
\begin{aligned}
\tilde{\varphi}_{\delta, \epsilon}(q, r) & =\int_{-1}^{1} q_{1}\left(-(1-\epsilon) \mathcal{L} r_{1}+\left(-(1-\epsilon) \tilde{\psi}(\delta, y)-\epsilon \frac{2 p(p+1)}{(p-1)^{2}} \frac{\left(1-\delta^{2}\right)}{(1+\delta y)^{2}}\right) r_{1}\right) \rho d y \\
& +(1-\epsilon) \int_{-1}^{1} q_{2} r_{2} \rho d y .
\end{aligned}
$$

To prove this proposition, we use the following:

Lemma 3.8 (Reduction of the proof of Proposition 3.7). There exists $\epsilon_{0} \in(0,1)$ such that for all $|\delta|<1$ and $r \in \tilde{\mathcal{H}}_{-}^{\delta}, \varphi_{\delta, \epsilon_{0}}(r, r) \geq 0$.

Lemma 3.8 implies Proposition 3.7 . As we have $|\tilde{\psi}(\delta, y)| \leq \frac{C}{1-y^{2}}$, we proceed exactly like in [33] page 91 .

Proof of Lemma 3.8. We proceed in three parts:

- In Part 1, we find a hyperplane of $\mathcal{H}$ where $\tilde{\varphi}_{\delta, \epsilon}$ is non-negative.

- In Part 2, we find a straight line in $\mathcal{H}$, where $\tilde{\varphi}_{\delta, \epsilon}$ is negative and which is "orthogonal" to $\tilde{\mathcal{H}}_{-}^{\delta}$ with respect to $\tilde{\varphi}_{\delta, \epsilon}$.

- In Part 3, we proceed by contradiction and prove that $\tilde{\varphi}_{\delta, \epsilon}$ is non-negative on $\tilde{\mathcal{H}}_{-}^{\delta}$.

Part $1: \tilde{\varphi}_{\delta, \epsilon}$ is non-negative on a hyperplane. We claim the following:

Lemma 3.9. There exists $\epsilon_{1}>0$ such that for all $|\delta|<1$ and $\epsilon \in\left(0, \epsilon_{1}\right], \tilde{\varphi}_{\delta, \epsilon}$ is non-negative on the hyperplane

$$
E_{1}=\left\{q \in \mathcal{H} \mid \int_{-1}^{1} \mathcal{T}_{-\delta}\left(q_{1}\right) \rho(y) d y=0\right\},
$$

where $\mathcal{T}_{-\delta}$ is defined in (176).

Proof. Define from (175) $\epsilon_{1}=\min \left(1, \frac{\gamma_{1}}{\gamma_{1}-\frac{2 p(p+1)}{(p-1)^{2}}}\right)>0$ and fix $\epsilon \in\left(0, \epsilon_{1}\right]$. We consider $u=\left(u_{1}, u_{2}\right) \in E_{1}$, and write from (59)

$$
\begin{aligned}
\tilde{\varphi}_{\delta, \epsilon}(u, u) & =\int_{-1}^{1} u_{1}\left(-(1-\epsilon) \mathcal{L} u_{1}+\left[-(1-\epsilon) \tilde{\psi}(\delta, y)-\epsilon \frac{2 p(p+1)}{(p-1)^{2}} \frac{\left(1-\delta^{2}\right)}{(1+\delta y)^{2}}\right] u_{1}\right) \rho(y) d y \\
& +(1-\epsilon) \int_{-1}^{1} u_{2}^{2} \rho(y) d y .
\end{aligned}
$$

If $U_{1}=\mathcal{T}_{-\delta} u_{1}$, then $u_{1}=\mathcal{T}_{\delta} U_{1}$ and we have from (176)

$$
\begin{aligned}
u_{1}(y)=\frac{\left(1-\delta^{2}\right)^{\frac{1}{p-1}}}{(1+\delta y)^{\frac{2}{p-1}}} U_{1}(z) \text { with } z & =\frac{y+\delta}{1+\delta y}, \\
\mathcal{L} u_{1}(y)+\tilde{\psi}(\delta, y) u_{1}(y) & =\frac{\left(1-\delta^{2}\right)^{\frac{p}{p-1}}}{(1+\delta y)^{\frac{2 p}{p-1}}} \mathcal{L} U_{1}(z), \\
\rho(y) d y & =\frac{(1+\delta y)^{\frac{2(p+1)}{p-1}}}{\left(1-\delta^{2}\right)^{\frac{p+1}{p-1}}} \rho(z) d z, \\
0 & =\int U_{1}(z) \rho(z) d z .
\end{aligned}
$$


Therefore, we see from (61) and Lemma C.4 that

$$
\begin{aligned}
\tilde{\varphi}_{\delta, \epsilon}(u, u)= & \int_{-1}^{1} U_{1}(z)\left(-(1-\epsilon) \mathcal{L} U_{1}(z)-\epsilon \frac{2 p(p+1)}{(p-1)^{2}} U_{1}(z)\right) \rho(z) d z \\
& +(1-\epsilon) \int_{-1}^{1} u_{2}^{2} \rho(y) d y \\
\geq & \left(-(1-\epsilon) \gamma_{1}-\epsilon \frac{2 p(p+1)}{(p-1)^{2}}\right) \int_{-1}^{1} U_{1}^{2}(z) \rho(z) d z+(1-\epsilon) \int_{-1}^{1} u_{2}^{2} \rho(y) d y \geq 0
\end{aligned}
$$

since $\epsilon \leq \epsilon_{1}$ hence $\left(-(1-\epsilon) \gamma_{1}-\epsilon \frac{2 p(p+1)}{(p-1)^{2}}\right) \geq 0$ and $1-\epsilon \geq 0$. This concludes the proof of Lemma 3.9

Part $2: \tilde{\varphi}_{\delta, \epsilon}$ is negative on a straight line orthogonal to $\tilde{\mathcal{H}}_{-}^{\delta}$. We need to find $\tilde{V}_{0}(\delta, \epsilon, \cdot)$ in $\mathcal{H}$ such that $\tilde{\varphi}_{\delta, \epsilon}\left(\tilde{V}_{0}(\delta, \epsilon, \cdot), r\right)=0$ for all $r \in \tilde{\mathcal{H}}_{-}^{\delta}$. Since we know from the definition of $\tilde{\mathcal{H}}_{-}^{\delta}$ (57) that

$$
\forall r \in \tilde{\mathcal{H}}_{-}^{\delta}, \phi\left(\tilde{W}_{0}, r\right)=\tilde{\pi}_{0}^{\delta}(r)=0,
$$

we proceed as on page 93 in [33] and search $\tilde{V}_{0}(\delta, \epsilon, \cdot)$ such that

$$
\forall r \in \mathcal{H}, \phi\left(\tilde{W}_{0}(\delta, \cdot), r\right)=\tilde{\varphi}_{\delta, \epsilon}\left(\tilde{V}_{0}(\delta, \epsilon, \cdot), r\right) .
$$

Then, we will show that $\tilde{\varphi}_{\delta, \epsilon}$ is negative on the straight line spanned by $\tilde{V}_{0}(\delta, \epsilon, \cdot)$. Consider $\epsilon>0$ small enough and take $|\delta|<1$. We claim the following:

Lemma 3.10. There exists $\epsilon_{2}>0$ such that for all $\epsilon \in\left(0, \epsilon_{2}\right)$ and $|\delta|<1$ :

(i) There exists $\tilde{V}_{0}(\delta, \epsilon, \cdot) \in \mathcal{H}_{0}$ such that (62) holds.

(ii) Moreover there exists $c>0$ such that

$$
\sup _{|\delta|<1}\left\|\epsilon \tilde{V}_{0}(\delta, \epsilon, \cdot)+c \tilde{F}_{0}(\delta, \cdot)\right\|_{\mathcal{H}} \rightarrow 0 \text { as } \epsilon \rightarrow 0^{+} .
$$

(iii) The bilinear form $\tilde{\varphi}_{\delta, \epsilon}$ is negative on a line of $\mathcal{H}$ spanned by $\tilde{V}_{0}(\delta, \epsilon, \cdot)$.

Proof of Lemma 3.10. We proceed in three steps:

-In Step 1, we find a PDE satisfied by $\tilde{V}_{0,1}(\delta, \epsilon, \cdot)$ and transform it with the Lorentz transform in similarity variables defined in (176).

In Step 2, we solve the transformed PDE and find the asymptotic behavior of $\tilde{V}_{0,1}(\delta, \epsilon, \cdot)$ as $\epsilon \rightarrow 0^{+}$, uniformly in $|\delta|<1$, which gives $(i)$ and $(i i)$.

In Step 3, we use that asymptotic behavior to show that $\tilde{\varphi}_{\delta, \epsilon}$ is negative on a straight line spanned by $\tilde{V}_{0}(\delta, \epsilon, \cdot)$, which gives $(i i i)$.

Step 1 (Reduction to the solution of some PDE). From the definition of $\tilde{\varphi}_{\delta, \epsilon}$ (59) and $\phi$ (35), we see that in order to satisfy (62), it is enough to take

$$
\tilde{V}_{0,2}(\delta, \epsilon, \cdot)=\tilde{W}_{0,2}(\delta, \cdot) /(1-\epsilon)
$$

and to prove the existence of a $\tilde{V}_{0,1}(\delta, \epsilon, \cdot)$ solution to

$$
\begin{aligned}
-(1-\epsilon) \mathcal{L} \tilde{V}_{0,1}(\delta, \epsilon, \cdot) & +\left(-(1-\epsilon) \tilde{\psi}(\delta, \cdot)-\epsilon \frac{2 p(p+1)}{(p-1)^{2}} \frac{\left(1-\delta^{2}\right)}{(1+\delta y)^{2}}\right) \tilde{V}_{0,1}(\delta, \epsilon, \cdot) \\
& =-\mathcal{L} \tilde{W}_{0,1}(\delta, \cdot)+\tilde{W}_{0,1}(\delta, \cdot) .
\end{aligned}
$$


Claim 3.11 (Reduction to an explicitly solvable PDE). Consider $\tilde{V}_{0,1}(\delta, \epsilon, \cdot)$ and introduce $\tilde{v}_{0,1}(\delta, \epsilon, \cdot)$ defined by

$$
\tilde{v}_{0,1}(\delta, \epsilon, \cdot)=\mathcal{T}_{-\delta} \tilde{V}_{0,1}(\delta, \epsilon, \cdot),
$$

where $\mathcal{T}_{\delta}$ is defined in (176). Then,

(i) $\tilde{V}_{0,1}(\delta, \epsilon, \cdot)$ is a solution to (65) if and only if $\tilde{v}_{0,1}(\delta, \epsilon, \cdot)$ is a solution to the equation

$$
\begin{aligned}
(1-\epsilon) \mathcal{L} \tilde{v}_{0,1}(\delta, \epsilon, z) & +\epsilon \frac{2 p(p+1)}{(p-1)^{2}} \tilde{v}_{0,1}(\delta, \epsilon, z)=f_{0}^{\delta}(z) \\
& \equiv \frac{1-\delta^{2}}{(1-\delta z)^{2}} \mathcal{T}_{-\delta}\left(\mathcal{L} \tilde{W}_{0,1}(\delta, y)-\tilde{W}_{0,1}(\delta, y)\right) .
\end{aligned}
$$

(ii) The linear form $h \mapsto \int_{-1}^{1} f_{0}^{\delta} h \rho$ defined for all $h \in \mathcal{H}_{0}$ is continuous and for some $C_{0}>0$, we have

$$
\forall \delta \in(-1,1),\left\|f_{0}^{\delta}\right\|_{\mathcal{H}_{0}^{\prime}} \leq C_{0}\left\|\tilde{W}_{0}(\delta, \cdot)\right\|_{\mathcal{H}} \leq C_{0}^{2} .
$$

Proof. (i) Using (176) we see that

$$
\begin{aligned}
& \tilde{V}_{0,1}(\delta, \epsilon, y)=\frac{\left(1-\delta^{2}\right)^{\frac{1}{p-1}}}{(1+\delta y)^{\frac{2}{p-1}}} \tilde{v}_{0,1}(\delta, \epsilon, z) \text { with } z=\frac{y+\delta}{1+\delta y}, \\
& \mathcal{L} \tilde{V}_{0,1}(\delta, \epsilon, y)+\tilde{\psi}(\delta, y) \tilde{V}_{0,1}(\delta, \epsilon, y)=\frac{\left(1-\delta^{2}\right)^{\frac{p}{p-1}}}{(1+\delta y)^{\frac{2 p}{p-1}}} \mathcal{L} \tilde{v}_{0,1}(\delta, \epsilon, z) .
\end{aligned}
$$

Since $\frac{(1-\delta z)^{2}}{1-\delta^{2}}=\frac{1-\delta^{2}}{(1+\delta y)^{2}}$, we see that equation (65) and (67) are equivalent.

(ii) The proof of $(i i)$ is the same as the proof of Claim 4.11 on page 94 in [33.

Step 2 (Solution of equation (67) and asymptotic behavior as $\epsilon \rightarrow 0^{+}$). We prove (i) and (ii) of Lemma 3.10 here. Let us first recall the following result from [33].

Claim 3.12 (Solution of equation (67) $)$. Consider

$$
f(y)=\sum_{n=0}^{\infty} \tilde{f}_{n} h_{n}(y) \in \mathcal{H}_{0}^{\prime},
$$

where $h_{n}$ are the eigenfunctions of $\mathcal{L}$ defined in Proposition C.3. Then, for any $\epsilon \in\left(0, \frac{1}{2}\right)$, the equation

$$
(1-\epsilon) \mathcal{L} v+\epsilon \frac{2 p(p+1)}{(p-1)^{2}} v=f
$$

has a unique solution in $\mathcal{H}_{0}$ given by

$$
v=\sum_{n=0}^{\infty} \frac{\tilde{f}_{n}}{\gamma_{n}+\left(\frac{2(p+1)}{(p-1)^{2}}-\gamma_{n}\right) \epsilon} h_{n},
$$

where $\gamma_{n} \leq 0$ are the eigenvalues of $\mathcal{L}$ introduced in Proposition C.3.

Now, we use this claim to prove $(i)$ and $(i i)$.

Proof of (i) of Lemma 3.10. Using (ii) in Claim 3.11, we see that $f_{0}^{\delta} \in \mathcal{H}_{0}^{\prime}$. Therefore, Claim 3.12 applies, and we have a unique solution $\tilde{v}_{0,2}(\delta, \epsilon, \cdot) \in \mathcal{H}_{0}$ to equation (67). Using $(i)$ of Lemma 3.4 and Lemma C.6 below we get a solution $\tilde{V}_{0,1}(\delta, \epsilon, \cdot) \in \mathcal{H}_{0}$ to equation (65). 
Proof of (ii) of Lemma 3.10. Note that the spectral properties of $\mathcal{L}$ are given in Proposition C.3 below. Since $h_{0}=c_{0}$ by Proposition C.3, we see from Claim 3.12 and (ii) in Claim 3.11 that for $\epsilon$ small enough,

$$
\left\|\tilde{v}_{0,1}(\delta, \epsilon, \cdot)-\frac{\tilde{f}_{0}}{\frac{2(p+1)}{(p-1)^{2}} \epsilon} c_{0}\right\|_{\mathcal{H}_{0}} \leq C\left\|f_{0}^{\delta}\right\|_{\mathcal{H}_{0}^{\prime}} \leq C,
$$

where from $(i i)$ in Lemma C.6 and the fact that $c_{0}=\mathcal{T}_{-\delta}\left(c_{0} \frac{\kappa(\delta, y)}{\kappa_{0}}\right)$ (see (176) $)$, we have

$$
\begin{aligned}
\tilde{f}_{0}^{\delta}=c_{0} \int_{-1}^{1} f(z) \rho(z) d z & =\frac{c_{0}}{\kappa_{0}} \int_{-1}^{1}\left(\mathcal{L} \tilde{W}_{0,1}(\delta, y)-\tilde{W}_{0,1}(\delta, y)\right) \kappa(\delta, y) \rho(y) d y \\
& =-\frac{c_{0}}{\kappa_{0}} \phi\left(\tilde{W}_{0}(\delta, \cdot), \tilde{F}_{0}(\delta, \cdot)\right)=-\frac{c_{0}}{\kappa_{0}}
\end{aligned}
$$

(use also the expression (35) of $\phi$ together with (42) and (49)). As $\tilde{V}_{0,2}(\delta, \epsilon, \cdot)$ is explicitly given by (64) and (47), we see that (63) follows from (66), (66), the fact that $\mathcal{T}_{\delta}\left(\kappa_{0}\right)=\kappa(\delta, y)$ and the expression of $\tilde{F}_{0}(42)$.

Step 3. Sign of $\tilde{\varphi}_{\delta, \epsilon}$ on the line spanned by $\tilde{V}_{0}(\delta, \epsilon, \cdot)$.

Proof of (iii) of Lemma 3.10. We will prove now that $\tilde{\varphi}_{\delta, \epsilon}$ is negative on the straight line spanned by $\tilde{V}_{0}(\delta, \epsilon, \cdot)$. From (62), (63) and (49), we see that

$$
\begin{aligned}
\tilde{\varphi}_{\delta, \epsilon}\left(\tilde{V}_{0}(\delta, \epsilon, \cdot), \tilde{V}_{0}(\delta, \epsilon, \cdot)\right) & =\phi\left(\tilde{W}_{0}(\delta, \cdot), \tilde{V}_{0}(\delta, \epsilon, \cdot)\right) \\
& \sim-\frac{c}{\epsilon} \phi\left(\tilde{W}_{0}(\delta, \cdot), \tilde{F}_{0}(\delta, \cdot)\right)=-\frac{c}{\epsilon}, \text { as } \epsilon \rightarrow 0,
\end{aligned}
$$

uniformly in $|\delta|<1$. So $\tilde{\varphi}_{\delta, \epsilon}\left(\tilde{V}_{0}(\delta, \epsilon, \cdot), \tilde{V}_{0}(\delta, \epsilon, \cdot)\right)<0$. This concludes the proof of Lemma 3.10.

Part 3 : End of the proof of Lemma 3.8. From Lemmas 3.9 and 3.10, we define $\epsilon_{0}=\min \left(\epsilon_{1}, \epsilon_{2}\right) \in(0,1)$. We will now prove by contradiction that $\tilde{\varphi}_{\delta, \epsilon_{0}}$ is positive on $\tilde{\mathcal{H}}_{-}^{\delta}$ for all $|\delta|<1$.

We note that from (57) and (62), for all $|\delta|<1$ and $\epsilon \in\left(0, \epsilon_{0}\right]$, the definition of $\tilde{\mathcal{H}}_{-}^{\delta}$ (57) is as follows:

$$
\tilde{\mathcal{H}}_{-}^{\delta}=\left\{r \in \mathcal{H} \mid \tilde{\varphi}_{\delta, \epsilon}\left(\tilde{V}_{0}(\delta, \epsilon, \cdot), r\right)=0\right\} .
$$

Consider $|\delta|<1$. By contradiction, assume that $\tilde{\varphi}_{\delta, \epsilon}$ is negative so

$$
\text { there is a non-zero } r \in \tilde{\mathcal{H}}_{-}^{\delta} \text { such that } \tilde{\varphi}_{\delta, \epsilon}(r, r)<0 \text {. }
$$

We mention that $r$ is not collinear $\tilde{V}_{0}(\delta, \epsilon, \cdot)$. Indeed, if $r=\alpha \tilde{V}_{0}(\delta, \epsilon, \cdot)$ with $\alpha \in \mathbb{R}^{*}$, then we would have

$$
\tilde{\varphi}_{\delta, \epsilon}\left(\tilde{V}_{0}(\delta, \epsilon, \cdot), r\right)=\alpha \tilde{\varphi}_{\delta, \epsilon}\left(\tilde{V}_{0}(\delta, \epsilon, \cdot), \tilde{V}_{0}(\delta, \epsilon, \cdot)\right) \neq 0,
$$

by (iii) in Lemma 3.10, which contradicts (69). Thus, the vector subspace

$$
E_{2}=\operatorname{span}\left(\tilde{V}_{0}(\delta, \epsilon, \cdot), r\right)
$$

is of dimension 2. Therefore, as the subspace $E_{1}$ (60) is of codimension 1 , there exists a non-zero $u \in E_{1} \cap E_{2}$.

On the one hand, since $u \in E_{1}$, we have from Lemma 3.9 that

$$
\tilde{\varphi}_{\delta, \epsilon}(u, u) \geq 0 .
$$


On the other hand, since $\tilde{\varphi}_{\delta, \epsilon}$ is negative on $E_{2}$ by $(i i i)$ of Lemma 3.10, we must have from (69) and (70),

$$
\tilde{\varphi}_{\delta, \epsilon}(u, u)<0 .
$$

This contradicts (171). So, $\tilde{\varphi}_{\delta, \epsilon}$ is non-negative on $\tilde{\mathcal{H}}_{-}^{\delta}$. This concludes the proof of Lemma 3.8 and Proposition 3.7 .

\section{TRAPPing NEAR THE SET OF STATIONARY SOLUtions}

In this part of the work in the real case in [33, the authors have assumed that (13) holds for some $s^{*} \in \mathbb{R}$ and $d^{*} \in(-1,1)$ and use modulation theory to introduce a parameter $d(s)$ adapted to the linearized equation and derive from the energy barrier the smallness of the unstable direction with respect to the stable. Then they use this to show that $\left(w(s), \partial_{s} w(s)\right)$ to some $\kappa\left(\delta_{\infty}, \cdot\right)$ as $s \rightarrow \infty$ in the norm of $\mathcal{H}$.

This section is devoted to the proofs of Proposition 3. Theorem 4 and Theorem 5. Let us first give the proof of Proposition 3 and then derive Theorem 5 from Theorem 4 . Later we prove Theorem 4.

4.1. Convergence to a stationary solution. We give the proofs of Proposition 3 and Theorem 5 here.

Proof of Proposition 3, From Proposition C.1 and Proposition 1, one can see that the proof given in the real case in Section 3.1 in [33] holds here with no change. Indeed, all the estimates remain valid in the complex case, in particular, the Sobolev embedding and the Duhamel formulation of the wave equation (11).

Proof of Theorem 5 assuming Theorem 4. Consider $w=w_{x_{0}}$ where $x_{0}$ is non-charteristic. The conclusion will follow from the application of Theorem 4 to $w_{x_{0}}$. In order to conclude, we have to check conditions (12) and (13). From the monotonicity of functional $E$ (See Proposition C.1 below) and (A.ii) of Proposition 3, we see that

$$
\forall s \geq-\log \left(T\left(x_{0}\right)\right), E\left(w(s), \partial_{s} w(s)\right) \geq E\left(\kappa_{0}, 0\right)
$$

and (12) follows. Consider $\epsilon^{*}$ defined in Theorem 4, From $(A . i)$ of Proposition 3 , we have the existence of $s^{*} \geq-\log T\left(x_{0}\right)$ such that

$$
\inf _{\{|\delta|<1, \theta \in \mathbb{R}\}}\left\|\left(\begin{array}{c}
w\left(s^{*}\right) \\
\partial_{s} w\left(s^{*}\right)
\end{array}\right)-e^{i \theta}\left(\begin{array}{c}
\kappa(\delta, \cdot) \\
0
\end{array}\right)\right\|_{H^{1} \times L^{2}} \leq \frac{\epsilon^{*}}{2} .
$$

Therefore, there exists $\left|\delta^{*}\right|<1$ and $\theta^{*} \in \mathbb{R}$ such that

$$
\left\|\left(\begin{array}{c}
w\left(s^{*}\right) \\
\partial_{s} w\left(s^{*}\right)
\end{array}\right)-e^{i \theta^{*}}\left(\begin{array}{c}
\kappa\left(\delta^{*}, \cdot\right) \\
0
\end{array}\right)\right\|_{H^{1} \times L^{2}} \leq \epsilon^{*} .
$$

Since $0 \leq \rho(y) \leq 1$, it follows that

$$
\inf _{\{|\delta|<1, \theta \in \mathbb{R}\}}\left\|\left(\begin{array}{c}
w\left(s^{*}\right) \\
\partial_{s} w\left(s^{*}\right)
\end{array}\right)-e^{i \theta}\left(\begin{array}{c}
\kappa(\delta, \cdot) \\
0
\end{array}\right)\right\|_{\mathcal{H}} \leq \epsilon^{*}
$$

and (13) follows. Applying Theorem 4, we get the conclusion of Theorem 5 
4.2. A modulation technique. We introduce two parameters $\delta(s)$ and $\theta(s)$ and we use a modulation technique to claim the following:

Proposition 4.1 (Modulation of $w$ with respect to $e^{i \theta} \kappa(\delta, \cdot)$ ). There exists $\epsilon_{1}>0$ and $K_{1}>0$ such that if $\left(w, \partial_{s} w\right) \in C\left(\left[s^{*}, \infty\right), \mathcal{H}\right)$ for some $s^{*} \in \mathbb{R}$ is a solution to equation (5) which satisfies (13) for some $\left|\delta^{*}\right|<1, \theta^{*} \in \mathbb{R}$ and $\epsilon^{*}<\epsilon_{1}$, then the following is true:

(i) (Choice of the modulation parameter) There exist $\delta(s) \in C^{1}\left(\left[s^{*}, \infty\right)\right.$, $(-1,1))$ and $\theta(s) \in C^{1}\left(\left[s^{*}, \infty\right), \mathbb{R}\right)$ such that for all $s \in\left[s^{*}, \infty\right)$,

$$
\check{\pi}_{0}^{\delta(s)}(\check{q}(s))=\tilde{\pi}_{0}^{\delta(s)}(\tilde{q}(s))=0
$$

where $\check{\pi}_{0}^{\delta}$ and $\tilde{\pi}_{0}^{\delta}$ are defined in (38), (55) and $q=\left(q_{1}, q_{2}\right)$ is defined for all $s \in$ $\left[s_{0}, \infty\right)$ by

$$
\left(\begin{array}{c}
w(y, s) \\
\partial_{s} w(y, s)
\end{array}\right)=e^{i \theta(s)}\left[\left(\begin{array}{c}
\kappa(\delta(s), y) \\
0
\end{array}\right)+\left(\begin{array}{c}
q_{1}(y, s) \\
q_{2}(y, s)
\end{array}\right)\right]
$$

Moreover,

$$
\left|\theta\left(s^{*}\right)-\theta^{*}\right|+\left|\log \left(\frac{1+\delta\left(s^{*}\right)}{1-\delta\left(s^{*}\right)}\right)-\log \left(\frac{1+\delta^{*}}{1-\delta^{*}}\right)\right|+\left\|q\left(s^{*}\right)\right\|_{\mathcal{H}} \leq K_{1} \epsilon^{*} .
$$

(ii) (Equation on $q$ ) For all $s \in\left[s^{*}, \infty\right)$,

$$
\begin{aligned}
& \frac{\partial}{\partial s}\left(\begin{array}{c}
\check{q}_{1} \\
\check{q}_{2}
\end{array}\right)=\check{L}_{\delta(s)}\left(\begin{array}{c}
\check{q}_{1} \\
\check{q}_{2}
\end{array}\right)+\left(\begin{array}{c}
0 \\
\check{f}_{\delta(s)}\left(q_{1}\right)
\end{array}\right)-\delta^{\prime}(s)\left(\begin{array}{c}
\partial_{\delta} \kappa(\delta, y) \\
0
\end{array}\right)+\theta^{\prime}(s)\left(\begin{array}{c}
\tilde{q}_{1} \\
\tilde{q}_{2}
\end{array}\right), \\
& \frac{\partial}{\partial s}\left(\begin{array}{c}
\tilde{q}_{1} \\
\tilde{q}_{2}
\end{array}\right)=\tilde{L}_{\delta(s)}\left(\begin{array}{c}
\tilde{q}_{1} \\
\tilde{q}_{2}
\end{array}\right)+\left(\begin{array}{c}
0 \\
\tilde{f}_{\delta(s)}\left(q_{1}\right)
\end{array}\right)-\theta^{\prime}(s)\left(\begin{array}{c}
\kappa(\delta, y)+\check{q}_{1} \\
\check{q}_{2}
\end{array}\right),
\end{aligned}
$$

where $\check{L}_{\delta(s)}, \tilde{L}_{\delta(s)}, \check{f}_{\delta(s)}$ and $\tilde{f}_{\delta(s)}$ are defined in (30), (32) and (34).

Proof. (i) From (38) and (56), we see that the condition (172) becomes

$$
\Phi\left(\left(w(s), \partial_{s} w(s)\right), \delta(s), \theta(s)\right)=0,
$$

where $\Phi \in C(\mathcal{H} \times(-1,1) \times \mathbb{R}, \mathbb{R} \times \mathbb{R})$ is defined by

$$
\Phi(v, \delta, \theta)=\left(\begin{array}{c}
\check{\Phi}(v, \delta, \theta) \\
\tilde{\Phi}(v, \delta, \theta)
\end{array}\right)=\left(\begin{array}{c}
\phi\left(\operatorname{Re}\left(e^{-i \theta} v-(\kappa(\delta, \cdot), 0)\right), \check{W}_{0}\right) \\
\phi\left(\operatorname{Im}\left(e^{-i \theta} v\right), \tilde{W}_{0}\right)
\end{array}\right) .
$$

We recall the following inequality which has been proved on page 102 in [33]:

$$
\forall \delta_{1}, \delta_{2} \in(-1,1),\left\|\kappa\left(\delta_{1}, \cdot\right)-\kappa\left(\delta_{2}, \cdot\right)\right\|_{\mathcal{H}_{0}} \leq C_{0}\left|\lambda_{1}-\lambda_{2}\right|, \text { where } \lambda_{i}=\log \left(\frac{1+\delta_{i}}{1-\delta_{i}}\right) .
$$

We apply the implicit function theorem to $\Phi$ near the point $\left(e^{i \theta^{*}}\left(\kappa\left(\delta^{*}, \cdot\right), 0\right), \delta^{*}, \theta^{*}\right)$.

Three facts have to be checked:

1-First, note that

$$
\Phi\left(e^{i \theta^{*}}\left(\kappa\left(\delta^{*}, \cdot\right), 0\right), \delta^{*}, \theta^{*}\right)=0 .
$$

2-Then, we compute from (76), for all $u \in \mathcal{H}$,

$$
\begin{aligned}
& D_{v} \check{\Phi}(v, \delta, \theta)(u)=\phi\left(\operatorname{Re}\left(e^{-i \theta} u\right), \check{W}_{0}\right), \\
& D_{v} \tilde{\Phi}(v, \delta, \theta)(u)=\phi\left(\operatorname{Im}\left(e^{-i \theta} u\right), \tilde{W}_{0}\right),
\end{aligned}
$$

so we have

$$
\left\|D_{v} \check{\Phi}(v, \delta, \theta)\right\| \leq C_{0} \text { and }\left\|D_{v} \tilde{\Phi}(v, \delta, \theta)\right\| \leq C_{0} .
$$


3-Let $J(\check{\Phi}, \tilde{\Phi})$ be the jacobian matrix of $\Phi$, and $D$ its determinant so

$$
J=\left(\begin{array}{cc}
\partial_{\delta} \check{\Phi} & \partial_{\theta} \check{\Phi} \\
\partial_{\delta} \tilde{\Phi} & \partial_{\theta} \tilde{\Phi}
\end{array}\right)
$$

where

$$
\begin{aligned}
\partial_{\delta} \check{\Phi} & =\phi\left(\left(\partial_{\delta} \kappa(\delta, \cdot), 0\right), \check{W}_{0}\right)+\phi\left(\operatorname{Re}\left(e^{-i \theta} v-(\kappa(\delta, \cdot), 0)\right), \partial_{\delta} \check{W}_{0}\right) \\
& =\frac{2 \kappa_{0}}{(p-1)\left(1-\delta^{2}\right)}+\phi\left(\operatorname{Re}\left(e^{-i \theta} v-(\kappa(\delta, \cdot), 0)\right), \partial_{\delta} \check{W}_{0}\right), \\
\partial_{\theta} \check{\Phi} & =\phi\left(\operatorname{Im}\left(e^{-i \theta} v\right), \check{W}_{0}\right), \\
\partial_{\delta} \tilde{\Phi} & =\phi\left(\operatorname{Im}\left(e^{-i \theta} v\right), \partial_{\delta} \tilde{W}_{0}\right), \\
\partial_{\theta} \tilde{\Phi} & =\phi\left(-\kappa(\delta, \cdot), \tilde{W}_{0}\right)+\phi\left(-\operatorname{Re}\left(e^{-i \theta} v\right)+\kappa(\delta, \cdot), \tilde{W}_{0}\right) \\
& =-1+\phi\left(-\operatorname{Re}\left(e^{-i \theta} v\right)+\kappa(\delta, \cdot), \tilde{W}_{0}\right),
\end{aligned}
$$

referring to Lemma 4.4 in 33 . for the first equation and the orthogonality relation (49) for the last one. Using the Cauchy-Schwarz inequality, the continuity of $\phi$ in $\mathcal{H}$, the bound (50), Lemma 4.4 in [33], and (77), we see that if

$$
\left|\theta-\theta^{*}\right|+\left|\log \left(\frac{1+\delta}{1-d}\right)-\log \left(\frac{1+\delta^{*}}{1-\delta^{*}}\right)\right|+\left\|v-e^{i \theta^{*}}\left(\kappa\left(\delta^{*}, \cdot\right), 0\right)\right\|_{\mathcal{H}} \leq \epsilon_{1}
$$

for some $\epsilon_{1}>0$ small enough independent of $\delta^{*}$, then we have

$$
\begin{aligned}
\mid \partial_{\delta} \check{\Phi} & -\frac{2 \kappa_{0}}{(p-1)\left(1-\delta^{2}\right)} \mid \leq \frac{C}{1-\delta^{2}}\left(\| \kappa\left(\delta^{*}, \cdot\right)-\kappa(\delta, \cdot)||_{\mathcal{H}_{0}}\right. \\
& \left.+\left\|\operatorname{Re}\left(e^{-i \theta^{*}} v-\left(\kappa\left(\delta^{*}, \cdot\right), 0\right)\right)\right\|_{\mathcal{H}}+\left\|\operatorname{Re}\left(v\left(e^{-i \theta}-e^{-i \theta^{*}}\right)\right)\right\|_{\mathcal{H}}\right) \leq \frac{C \epsilon_{1}}{1-\delta^{2}}
\end{aligned}
$$

$$
\begin{gathered}
\left|\partial_{\delta} \tilde{\Phi}\right| \leq \frac{C}{1-\delta^{2}}\left\|\operatorname{Im}\left(e^{-i \theta} v\right)\right\|_{\mathcal{H}} \leq \frac{C \epsilon_{1}}{1-\delta^{2}}, \\
\left|\partial_{\theta} \check{\Phi}\right| \leq C\left\|\operatorname{Im}\left(e^{-i \theta} v\right)\right\|_{\mathcal{H}} \leq C \epsilon_{1},
\end{gathered}
$$

$$
\begin{aligned}
\left|\partial_{\theta} \tilde{\Phi}+1\right| & =\left|\phi\left(-\operatorname{Re}\left(e^{-i \theta} v\right)+\kappa(\delta, \cdot), \tilde{W}_{0}\right)\right| \leq C\left(\|-\operatorname{Re}\left(e^{-i \theta^{*}} v\right)\right. \\
& \left.+\kappa\left(\delta^{*}, \cdot\right)\left\|_{\mathcal{H}}+\right\| \operatorname{Re}\left(v\left(e^{-i \theta}-e^{-i \theta^{*}}\right)\right)\left\|_{\mathcal{H}}+\right\| \kappa(\delta, \cdot)-\kappa\left(\delta^{*}, \cdot\right) \|_{\mathcal{H}_{0}}\right) \leq C \epsilon_{1} .
\end{aligned}
$$

Collecting (80)- (83), we see that for $\epsilon_{1}$ small enough, we have

$$
\left|D+\frac{2 \kappa_{0}}{(p-1)\left(1-\delta^{2}\right)}\right| \leq \frac{C \epsilon_{1}}{1-\delta^{2}},
$$

so we have the non-degeneracy of $\tilde{\Phi}$ near the point $\left(e^{i \theta^{*}}\left(\kappa\left(\delta^{*}, \cdot\right), 0\right), \delta^{*}, \theta^{*}\right)$. Applying the implicit function theorem, we see from (78) and (84) that there exist $\epsilon_{2}, \epsilon_{3}>0, C^{1}$ applications $(f, g): \mathcal{H} \rightarrow(-1,1) \times \mathbb{R}$ such that for all $v \in \mathcal{H}$ satisfying $\left\|e^{i \theta^{*}}\left(\kappa\left(\delta^{*}, \cdot\right), 0\right)-v\right\|_{\mathcal{H}} \leq \epsilon_{2}$ and for all $(\delta, \theta) \in(-1,1) \times \mathbb{R}$ satisfying $\left|\delta-\delta^{*}\right|+$ $\left|\theta-\theta^{*}\right| \leq \epsilon_{3}$ we have

$$
\Phi(v, \delta, \theta)=0 \Leftrightarrow(\delta, \theta)=(f(v), g(v)) .
$$


Take $\epsilon_{0}=\frac{\epsilon_{2}}{2}$ and consider $\epsilon^{*} \leq \epsilon_{0}$. From (13) and the continuity of $\left(w, \partial_{s} w\right)$, we see that for some $\sigma^{*}>s^{*}$, we have:

$$
\forall s \in\left[s^{*}, \sigma^{*}\right],\left\|\left(w(s), \partial_{s} w(s)\right)-e^{i \theta^{*}}\left(\kappa\left(\delta^{*}, \cdot\right), 0\right)\right\|_{\mathcal{H}} \leq 2 \epsilon^{*} \leq \epsilon_{2} .
$$

Therefore, from (76) and (85), we see that requiring (72) is equivalent to having $d=f(w(s))$ and $\theta=g(w(s))$. Since $f$ and $g$ are $C^{1}$, we get the conclusion with $C^{1}$ functions $\delta(s)$ and $\theta(s)$ such that (72) holds for all $s \in\left[s^{*}, \sigma^{*}\right]$.

Now, let's prove that $\sigma^{*}=+\infty$. By contradiction, suppose $\sigma^{*}<+\infty$. We apply the implicit function theorem to $\Phi$ at the point $\left(v_{n}, \delta_{n}, \theta_{n}\right) \equiv\left(\left(w\left(s_{n}\right), \partial_{s} w\left(s_{n}\right)\right)\right.$, $\left.\delta\left(s_{n}\right), \theta\left(s_{n}\right)\right)$ where $s_{n}=\sigma^{*}-\frac{1}{n}$, and the uniform continuity of $\left(w(s), \partial_{s} w(s)\right)$ from $\left[\sigma^{*}-\eta_{0}, \sigma^{*}+\eta_{0}\right]$ to $\mathcal{H}$ for some $\eta_{0}>0$. In fact, from (85), $\Phi\left(\left(w\left(s_{n}\right), \partial_{s} w\left(s_{n}\right)\right), \delta\left(s_{n}\right)\right.$, $\left.\theta\left(s_{n}\right)\right)=0$. Moreover, (78) and (84) are uniformly satisfied, so as above we see that we can define $\delta(s)$ for all $s \in\left[s_{n}, s_{n}+\epsilon_{0}\right]$ for some $\epsilon_{0}>0$ independent of $n$. Therefore, for $n$ large enough, $\delta(s)$ exists beyond $\sigma^{*}$, which is a contradiction. Thus, $\sigma^{*}=+\infty$.

(ii) This is a direct consequence of the equation (5) satisfied by $w$ put in vectorial form:

$$
\begin{aligned}
\partial_{s} w & =v \\
\partial_{s} v & =\mathcal{L} w-\frac{2(p+1)}{(p-1)^{2}} w+|w|^{p-1} w-\frac{p+3}{p-1} v-2 y \partial_{y} v
\end{aligned}
$$

and the fact that $(\kappa(\delta, \cdot), 0)$ satisfies

$$
\mathcal{L} \kappa(\delta, \cdot)-\frac{2(p+1)}{(p-1)^{2}} \kappa(\delta, \cdot)+|\kappa(\delta, \cdot)|^{p-1} \kappa(\delta, \cdot)=0
$$

as a stationary solution. We have from (73)

$$
\begin{aligned}
\partial_{s} q_{1} & =q_{2}-i \theta^{\prime}\left(\kappa(\delta(s), y)+q_{1}\right)-\delta^{\prime} \partial_{\delta} \kappa \\
\partial_{s} q_{2} & =\mathcal{L} \kappa(\delta(s), y)+\mathcal{L} q_{1}-\frac{2(p+1)}{(p-1)^{2}}\left(\kappa(\delta(s), y)+q_{1}\right) \\
& +\left|\kappa(\delta(s), y)+q_{1}\right|^{p-1}\left(\kappa(\delta(s), y)+q_{1}\right)-\frac{p+3}{p-1} q_{2}-2 y \partial_{y} q_{2}-i \theta^{\prime} q_{2} .
\end{aligned}
$$

Dissociating the real and the imaginary part of these equations, we get (74) and (75).

4.3. Projection on the eigenspaces of the operator $L_{\delta}$. Given $s \geq s^{*}$ and following the previous section, we make in this section the following a priori estimate

$$
\|q(s)\|_{\mathcal{H}} \leq \epsilon
$$

for some $\epsilon>0$. From (72), we will expand $\check{q}$ and $\tilde{q}$ respectively according to the spectrum of the linear operators $\check{L}_{\delta}$ and $\tilde{L}_{\delta}$ as in (39) and (56):

$$
\begin{aligned}
& \check{q}(y, s)=\check{\alpha}_{1} \check{F}_{1}(\delta, y)+\check{q}_{-}(y, s), \\
& \tilde{q}(y, s)=\tilde{q}_{-}(y, s),
\end{aligned}
$$

where

$$
\begin{gathered}
\check{\alpha}_{1}=\check{\pi}_{1}^{\delta(s)}(\check{q}), \check{\alpha}_{0}=\check{\pi}_{0}^{\delta(s)}(\check{q})=0, \check{\alpha}_{-}(s)=\sqrt{\check{\varphi}_{\delta}\left(\check{q}_{-}, \check{q}_{-}\right)}, \\
\tilde{\alpha}_{0}=\tilde{\pi}_{0}^{\delta(s)}(\tilde{q})=0, \tilde{\alpha}_{-}(s)=\sqrt{\tilde{\varphi}_{\delta}\left(\tilde{q}_{-}, \tilde{q}_{-}\right)}
\end{gathered}
$$


and

$$
\begin{aligned}
& \check{q}_{-}=\left(\begin{array}{c}
\check{q}_{-, 1} \\
\check{q}_{-, 2}
\end{array}\right)=\check{\pi}_{-}^{\delta}(\check{q})=\check{\pi}_{-}^{\delta}\left(\begin{array}{c}
\check{q}_{1} \\
\check{q}_{2}
\end{array}\right), \\
& \tilde{q}_{-}=\left(\begin{array}{c}
\tilde{q}_{-, 1} \\
\tilde{q}_{-, 2}
\end{array}\right)=\tilde{\pi}_{-}^{\delta}(\tilde{q})=\tilde{\pi}_{-}^{\delta}\left(\begin{array}{c}
\tilde{q_{1}} \\
\tilde{q}_{2}
\end{array}\right) .
\end{aligned}
$$

From (87), (88), (41) and Proposition 3.7, we see that for all $s \geq s_{0}$,

$$
\begin{aligned}
\frac{1}{C_{0}} \check{\alpha}_{-}(s) & \leq\left\|\check{q}_{-}(s)\right\|_{\mathcal{H}} \leq C_{0} \check{\alpha}_{-}(s), \\
\frac{1}{C_{0}}\left(\left|\check{\alpha}_{1}(s)\right|+\check{\alpha}_{-}(s)\right) & \leq\|\check{q}(s)\|_{\mathcal{H}} \leq C_{0}\left(\left|\check{\alpha}_{1}(s)\right|+\check{\alpha}_{-}(s)\right), \\
\frac{1}{C_{0}} \tilde{\alpha}_{-}(s) & \leq\|\tilde{q}(s)\|_{\mathcal{H}} \leq C_{0} \tilde{\alpha}_{-}(s),
\end{aligned}
$$

for some $C_{0}>0$. Let us introduce

$$
R_{-}(s)=-\int_{-1}^{1} \mathcal{F}_{\delta}\left(q_{1}\right) \rho d y,
$$

where

$$
\begin{aligned}
\mathcal{F}_{\delta(s)}\left(q_{1}(y, s)\right)= & \frac{\left|\kappa(\delta, \cdot)+q_{1}\right|^{p+1}}{p+1}-\frac{\kappa(\delta, \cdot)^{p+1}}{p+1} \\
& -\kappa(\delta, \cdot)^{p} \check{q}_{1}-\frac{p}{2} \kappa(\delta, \cdot)^{p-1} \check{q}_{1}^{2}-\frac{\kappa(\delta, \cdot)^{p-1}}{2} \tilde{q}_{1}^{2} .
\end{aligned}
$$

In the following proposition, we derive from (74) and (75) differential inequalities satisfied by $\check{\alpha}_{1}(s), \check{\alpha}_{-}(s), \tilde{\alpha}_{-}(s), \theta(s)$ and $\delta(s)$.

Proposition 4.2. There exists $C_{0}$ and $\epsilon_{2}>0$ such that if $w$ is a solution to equation (5) satisfying (72) and (86) at some time $s$ for some $\epsilon \leq \epsilon_{2}$, where $q$ is defined in (173), then:

\section{(i) (Control of the modulation parameter)}

$$
\left|\theta^{\prime}\right|+\frac{\left|\delta^{\prime}\right|}{1-\delta^{2}} \leq C_{0}\left(\check{\alpha}_{1}^{2}+\check{\alpha}_{-}^{2}+\tilde{\alpha}_{-}^{2}\right) .
$$

(ii) (Projection of equation (174) on the different eigenspaces of $\check{L}_{\delta}$ and $\left.\tilde{L}_{\delta}\right)$

$$
\left|\check{\alpha}_{1}^{\prime}-\check{\alpha}_{1}\right| \leq C_{0}\left(\check{\alpha}_{1}^{2}+\check{\alpha}_{-}^{2}+\tilde{\alpha}_{-}^{2}\right)
$$

$$
\left(R_{-}+\frac{1}{2}\left(\check{\alpha}_{-}^{2}+\tilde{\alpha}_{-}^{2}\right)\right)^{\prime} \leq-\frac{4}{p-1} \int_{-1}^{1}\left(\check{q}_{-, 2}^{2}+\tilde{q}_{-, 2}^{2}\right) \frac{\rho}{1-y^{2}} d y+C_{0}\left(\check{\alpha}_{1}^{2}+\check{\alpha}_{-}^{2}+\tilde{\alpha}_{-}^{2}\right)^{\frac{3}{2}},
$$

for $R_{-}(s)$, as defined in (92), satisfying

$$
\left|R_{-}(s)\right| \leq C_{0}\left(\check{\alpha}_{1}^{2}+\check{\alpha}_{-}^{2}+\tilde{\alpha}_{-}^{2}\right)^{\frac{1+\check{p}}{2}}, \text { where } \check{p}=\min (p, 2)>1 \text {. }
$$

(iii) (An additional relation)

$$
\begin{aligned}
& \frac{d}{d s} \int_{-1}^{1} \check{q}_{1} \check{q}_{2} \rho \leq-\frac{4}{5} \check{\alpha}_{-}^{2}+C_{0} \int_{-1}^{1} \check{q}_{-, 2}^{2} \frac{\rho}{1-y^{2}}+C_{0}\left(\check{\alpha}_{1}^{2}+\tilde{\alpha}_{-}^{2}\right), \\
& \frac{d}{d s} \int_{-1}^{1} \tilde{q}_{1} \tilde{q}_{2} \rho \leq-\frac{4}{5} \tilde{\alpha}_{-}^{2}+C_{0} \int_{-1}^{1} \tilde{q}_{2}^{2} \frac{\rho}{1-y^{2}}+C_{0}\left(\check{\alpha}_{1}^{2}+\check{\alpha}_{-}^{2}\right) .
\end{aligned}
$$


(iv) (Energy barrier) If moreover (12) holds, then

$$
\check{\alpha}_{1}(s) \leq C_{0} \check{\alpha}_{-}(s)+C_{1} \tilde{\alpha}_{-}(s) \text {. }
$$

Remark. Estimate (96) shows a kind of Lyapunov functional for system (74)-(75). Indeed, if we imagine for a second that $\delta$ and $\theta$ do not depend on $s$ (in other words, if we forget the modulation technique), then proving (96) reduces to finding a Lyapunov functional for system (74)-(75), which follows, as for equation (5), by multiplication by the conjugate of the time derivative and then by integration over $(-1,1)$.

Because of the modulation, we need to be more careful and use $(i)$ to show that $\left|\delta^{\prime}\right|$ and $\left|\theta^{\prime}\right|$ are quadratic in $\|q\|_{\mathcal{H}}$.

Remark. The estimates concerning $\theta^{\prime}(s)$ and $R_{-}$are among the novelties of our paper, since they directly involve the complex structure. The other estimates are parallel to those of the real case treated in [33].

Remark. The behavior of the solution will be derived in Section 4.4 below, thanks to the differential inequalities stated in Proposition 4.2 above. One issue will be to show that the unstable direction $\check{\alpha}_{1}$, which satisfies (95) never dominates the other components. This fact is true from (100), which is a direct consequence of the energy barrier hypothesis $E\left(w(s), \partial_{s} w(s)\right) \geq E\left(\kappa_{0}, 0\right)$ given in (12). Let us stress the fact that such a hypothesis is natural, since Theorem 4 will be applied with $w=w_{x_{0}}$ where $x_{0}$ is non-characteristic, and thanks to Proposition 3 we know that (100) holds.

Let us give the following estimate:

Claim 4.3. For all $y \in(-1,1), q_{1}=\check{q}_{1}+i \tilde{q}_{1}$

$$
\begin{aligned}
& \left|f_{\delta(s)}\left(q_{1}(y, s)\right)\right| \leq C_{0} m M\left(k(\delta(s), y)^{p-2}\left|q_{1}(y, s)\right|^{2},\left|q_{1}(y, s)\right|^{p}\right), \\
& \left|\mathcal{F}_{\delta(s)}\left(q_{1}(y, s)\right)\right| \leq C_{0} m M\left(k(\delta(s), y)^{p-2}\left|q_{1}(y, s)\right|^{3},\left|q_{1}(y, s)\right|^{p+1}\right),
\end{aligned}
$$

where $f_{\delta}\left(q_{1}\right)$ and $\mathcal{F}_{\delta}\left(q_{1}\right)$ are introduced in (30) and (93), $m M=\min$ if $1<p<2$ and $m M=\max$ if $p \geq 2$.

Proof of Claim 4.3. Introducing $\xi=\check{\xi}+i \tilde{\xi}=q_{1} / \kappa(\delta(s), y)$ and considering the cases $|\xi| \leq 1$ and $|\xi| \geq 1$, for $f_{\delta}\left(q_{1}\right)=\check{f}_{\delta}\left(q_{1}\right)+i \tilde{f}_{\delta}\left(q_{1}\right)$ given in (30), we get the conclusion.

Proof of Proposition 4.2. We proceed in four parts in order to prove Proposition 4.2 ;

-In Part 1, we project equations (74) and (75) respectively with the projectors $\check{\pi}_{\lambda}(\lambda \in\{0,1\})$ (38) and $\tilde{\pi}_{0}$ (55) and we derive the smallness condition on $\delta^{\prime}$ and $\theta^{\prime}$, together with (95).

-In Part 2, we first give some preliminary estimations; then from the derivatives of $\check{\alpha}_{-}^{2}$ and $\tilde{\alpha}_{-}^{2}$ given by the quadratic form $\check{\phi}_{\delta}$ and $\tilde{\phi}_{\delta}$, we get (96) and (97).

-In Part 3, writing equations satisfied by $\check{q}(74), \tilde{q}(75)$ and using (72) we prove (98) and (99).

-In Part 4, we prove (iv).

Part 1 : Projection of equations (74) and (75). Projecting equation (74) with the projector $\check{\pi}_{\lambda}^{\delta}$ (38) for $\lambda=0$ and $\lambda=1$, we write

$$
\check{\pi}_{\lambda}^{\delta}\left(\partial_{s} \check{q}\right)=\check{\pi}_{\lambda}^{\delta}\left(\check{L}_{\delta(s)} \check{q}\right)+\check{\pi}_{\lambda}^{\delta}\left(\begin{array}{c}
0 \\
\check{f}_{\delta(s)}\left(q_{1}\right)
\end{array}\right)-\delta^{\prime}(s) \check{\pi}_{\lambda}^{\delta}\left(\begin{array}{c}
\partial_{\delta} \kappa(\delta, y) \\
0
\end{array}\right)+\theta^{\prime}(s) \check{\pi}_{\lambda}^{\delta}\left(\begin{array}{c}
\tilde{q}_{1} \\
\tilde{q}_{2}
\end{array}\right) .
$$


Proceeding exactly as on page 105 in 33 , with (86) and (91), and using the fact that

we get

$$
\left|\check{\pi}_{\lambda}^{\delta}\left(\begin{array}{c}
\tilde{q_{1}} \\
\tilde{q}_{2}
\end{array}\right)\right|=\left|\phi\left(\check{W}_{\lambda}(\delta, \cdot),\left(\begin{array}{c}
\tilde{q}_{1} \\
\tilde{q_{2}}
\end{array}\right)\right)\right| \leq\left\|\check{W}_{\lambda}(\delta, \cdot)\right\|\left\|_{\mathcal{H}}|| \tilde{q}\right\|_{\mathcal{H}} \leq C \tilde{\alpha}_{-},
$$

$$
\begin{array}{r}
\frac{2 \kappa_{0}}{(p-1)\left(1-\delta^{2}\right)}\left|\delta^{\prime}\right| \leq \frac{C_{0}}{1-\delta^{2}}\left|\delta^{\prime}\right|\left(\left|\check{\alpha}_{1}\right|+\check{\alpha}_{-}\right)+C_{0}\left(\check{\alpha}_{1}^{2}+\check{\alpha}_{-}^{2}+\tilde{\alpha}_{-}^{2}\right)+C_{0}\left|\theta^{\prime}\right| \tilde{\alpha}_{-}, \\
(104) \quad\left|\check{\alpha}_{1}^{\prime}(s)-\check{\alpha}_{1}(s)\right| \leq \frac{C_{0}}{1-\delta^{2}}\left|\delta^{\prime}\right|\left(\left|\check{\alpha}_{1}\right|+\check{\alpha}_{-}\right)+C_{0}\left(\check{\alpha}_{1}^{2}+\check{\alpha}_{-}^{2}+\tilde{\alpha}_{-}^{2}\right)+C_{0}\left|\theta^{\prime}\right| \tilde{\alpha}_{-} .
\end{array}
$$

Now, projecting equation (75) with the projector $\tilde{\pi}_{0}^{\delta}$ (55), we get

$$
\tilde{\pi}_{0}^{\delta}\left(\partial_{s} \tilde{q}\right)=\tilde{\pi}_{0}^{\delta}\left(\tilde{L}_{\delta(s)} \tilde{q}\right)+\tilde{\pi}_{0}^{\delta}\left(\begin{array}{c}
0 \\
\tilde{f}_{\delta(s)}\left(q_{1}\right)
\end{array}\right)-\theta^{\prime}(s) \tilde{\pi}_{0}^{\delta}\left(\begin{array}{c}
\kappa(\delta, y)+\check{q_{1}} \\
\check{q_{2}}
\end{array}\right)
$$

Since $\tilde{\alpha}_{0}(s)=\tilde{\pi}_{0}^{\delta}(\tilde{q})=\phi\left(\tilde{W}_{0}(\delta, \cdot), \tilde{q}\right)=0$ by (90) and the definition of $\tilde{\pi}_{0}^{\delta}(\underline{55)}$, we write

$$
0=\tilde{\alpha}_{0}^{\prime}(s)=\tilde{\pi}_{0}^{\delta}\left(\partial_{s} \tilde{q}\right)+\delta^{\prime}(s) \tilde{\phi}\left(\partial_{\delta} \tilde{W}_{0}(\delta, \cdot), \tilde{q}\right) .
$$

Using (50) and (91), we get

$$
\left|\tilde{\pi}_{0}^{\delta}\left(\partial_{s} \tilde{q}\right)\right| \leq \frac{C_{0}}{1-\delta^{2}}\left|\delta^{\prime}\right| \tilde{\alpha}_{-} .
$$

Using $(i)$ of Lemma 3.4, the definition of $\tilde{\pi}_{0}^{\delta}$ (155), we write

$$
\tilde{\pi}_{0}^{\delta}\left(\tilde{L}_{\delta}(\tilde{q})\right)=\tilde{\phi}\left(\tilde{W}_{0}(\delta, \cdot), \tilde{L}_{\delta}(\tilde{q})\right)=\tilde{\phi}\left(\tilde{L}_{\delta}^{*}\left(\tilde{W}_{0}(\delta, \cdot)\right), \tilde{q}\right)=0 .
$$

From the definitions of $\tilde{\pi}_{0}^{\delta}(55)$ and $\phi(35)$, together with Claim 4.3, we see that

$$
\begin{aligned}
& \left|\tilde{\pi}_{0}^{\delta}\left(\begin{array}{c}
0 \\
\tilde{f}_{\delta(s)}\left(q_{1}\right)
\end{array}\right)\right| \leq C \int_{-1}^{1} \kappa(\delta, y)\left|\tilde{f}_{\delta}\left(q_{1}\right)\right| \rho(y) d y \\
& \leq C_{0} \int_{-1}^{1} \kappa(\delta, y)^{p-1}\left|q_{1}(y, s)\right|^{2} \rho d y+C_{0} \delta_{\{p \geq 2\}} \int_{-1}^{1} \kappa(\delta, y)\left|q_{1}(y, s)\right|^{p} \rho(y) d y \\
& \leq C_{0}\left\|q_{1}\right\|_{L_{\rho}^{p+1}}^{2}\left\|\left.\kappa(\delta, \cdot)\right|_{L_{\rho}^{p+1}} ^{p-1}+C_{0} \delta_{\{p \geq 2\}}\right\| q_{1}\left\|_{L_{\rho}^{p+1}}^{p}\right\| \kappa(\delta, \cdot) \|_{L_{\rho}^{p+1}},
\end{aligned}
$$

where $\delta_{\{p \geq 2\}}$ is 0 if $1<p<2$ and 1 otherwise. Therefore, using (108), Lemma C.2. (86) and (91), we get

$$
\left|\tilde{\pi}_{0}^{\delta}\left(\begin{array}{c}
0 \\
\tilde{f}_{\delta(s)}\left(q_{1}\right)
\end{array}\right)\right| \leq C_{0}\left(\check{\alpha}_{1}(s)^{2}+\check{\alpha}_{-}(s)^{2}+\tilde{\alpha}_{-}(s)^{2}\right) .
$$

Since $\tilde{\pi}_{0}^{\delta}\left(\begin{array}{c}\kappa(\delta, y) \\ 0\end{array}\right)=1$ from Lemmas 3.1 and 3.4 and using (50) and (91), we write

$$
\left|\tilde{\pi}_{0}^{\delta}\left(\begin{array}{c}
\kappa(\delta, y)+\check{q}_{1} \\
\check{q}_{2}
\end{array}\right)-1\right|=\left|\tilde{\pi}_{0}^{\delta}\left(\begin{array}{c}
\kappa(\delta, y)+\check{q}_{1} \\
\check{q}_{2}
\end{array}\right)-\tilde{\pi}_{0}^{\delta}\left(\begin{array}{c}
\kappa(\delta, y) \\
0
\end{array}\right)\right| \leq C_{0}\left(\left|\check{\alpha}_{1}(s)\right|+\check{\alpha}_{-}(s)\right) .
$$

Using (106), (107), (109) and (110), to bound the terms of equation (105) we get:

$$
\left|\theta^{\prime}\right| \leq \frac{C_{0}}{1-\delta^{2}}\left|\delta^{\prime}\right| \tilde{\alpha}_{-}+C_{0}\left(\check{\alpha}_{1}^{2}+\check{\alpha}_{-}^{2}+\tilde{\alpha}_{-}^{2}\right)+C_{0}\left|\theta^{\prime}\right|\left(\left|\check{\alpha}_{1}\right|+\check{\alpha}_{-}\right) .
$$


Using (86) and (91), we see that

hence

$$
\left|\theta^{\prime}\right|+\frac{2 \kappa_{0}}{p-1} \frac{\left|\delta^{\prime}\right|}{1-\delta^{2}} \leq C_{0} \epsilon \frac{\left|\delta^{\prime}\right|}{1-\delta^{2}}+C_{0}\left(\check{\alpha}_{1}^{2}+\check{\alpha}_{-}^{2}+\tilde{\alpha}_{-}^{2}\right)+C_{0} \epsilon\left|\theta^{\prime}\right|,
$$

$$
\left(1-C_{0} \epsilon\right)\left|\theta^{\prime}\right|+\left(\frac{2 \kappa_{0}}{p-1}-C_{0} \epsilon\right) \frac{\left|\delta^{\prime}\right|}{1-\delta^{2}} \leq C_{0}\left(\check{\alpha}_{1}^{2}+\check{\alpha}_{-}^{2}+\tilde{\alpha}_{-}^{2}\right) .
$$

Taking $\epsilon$ small enough, we get (94). Then using (94) to bound the term of the right hand side of (104) we get (95).

Part 2 : A kind of Lyapunov functional for system (74)-(75). We need to put together information from $\check{q}_{-}$and $\tilde{q}_{-}$in order to conclude. Handling each one alone doesn't allow us to control the terms $\int_{-1}^{1} \check{q}_{2} \check{f}_{\delta}\left(q_{1}\right) \rho d y$ and $\int_{-1}^{1} \tilde{q}_{2} \tilde{f}_{\delta}\left(q_{1}\right) \rho d y$ which appear in the differential inequalities satisfied by $\check{\alpha}_{-}$and $\tilde{\alpha}_{-}$(use (115) and (121) below). We claim that (96) follows from the following lemmas:

Lemma 4.4 (Preliminary estimates for $\left.\check{q}_{-}\right)$). There exists $\epsilon_{3}>0$ such that if $\epsilon \leq \epsilon_{3}$ in the hypotheses of Proposition 4.2, then

$$
\begin{array}{r}
\left\|\partial_{s} \check{q}_{-}-\check{L}_{\delta}\left(\check{q}_{-}\right)-\check{\pi}_{-}^{\delta}\left(\begin{array}{c}
0 \\
\check{f}_{\delta}\left(q_{1}\right)
\end{array}\right)\right\|_{\mathcal{H}} \leq C_{0}\left(\check{\alpha}_{1}^{2}+\check{\alpha}_{-}^{2}+\tilde{\alpha}_{-}^{2}\right)^{\frac{3}{2}}, \\
\left|\check{\varphi}_{\delta}\left(\check{q}_{-}, \check{\pi}_{-}^{\delta}\left(\begin{array}{c}
0 \\
\check{f}_{\delta}\left(q_{1}\right)
\end{array}\right)\right)-\int_{-1}^{1} \check{q}_{2} \check{f}_{\delta}\left(q_{1}\right) \rho d y\right| \leq C_{0}\left(\check{\alpha}_{1}^{2}+\check{\alpha}_{-}^{2}+\tilde{\alpha}_{-}^{2}\right)^{\frac{3}{2}} .
\end{array}
$$

Proof. Since the equation (74) satisfied by $\check{q}$ is the same as in the real case treated in [33], except for the last term $\theta^{\prime}\left(\tilde{q}_{1}, \tilde{q}_{2}\right)$, we refer the reader to Claim 5.4 on page 106 in [33, and focus only on the last term. Using (91) and (94), we see that

$$
\left\|\theta^{\prime}\left(\tilde{q}_{1}, \tilde{q}_{2}\right)\right\|_{\mathcal{H}} \leq C_{0}\left(\check{\alpha}_{1}^{2}+\check{\alpha}_{-}^{2}+\tilde{\alpha}_{-}^{2}\right)^{\frac{3}{2}},
$$

which is precisely the error in the right hand sides of the inequalities in Lemma 4.4 , Since our equation (5) satisfies (86) and (91), we see that the proof of Merle and Zaag on page 108 in 33. can be adapted in our case.

Lemma 4.5 (Preliminary estimates for $\tilde{q}_{-}$). There exists $\epsilon_{4}>0$ such that if $\epsilon \leq \epsilon_{4}$ in the hypotheses of Proposition 4.2, then

$$
\left|\int_{-1}^{1} \check{q}_{2} \check{f}_{\delta}\left(q_{1}\right) \rho d y+\int_{-1}^{1} \tilde{q}_{2} \tilde{f}_{\delta}\left(q_{1}\right) \rho d y-\frac{d}{d s} \int_{-1}^{1} \mathcal{F}_{\delta(s)} \rho d y\right| \leq C_{0}\left(\check{\alpha}_{1}^{2}+\check{\alpha}_{-}^{2}+\tilde{\alpha}_{-}^{2}\right)^{2} .
$$

Remark. Note that (114) is one of the new features of our paper. Indeed, it directly involves the complex structure.

Let us derive (96) and (97) from Lemmas 4.4 and 4.5. Then we prove Lemma 4.5 .

Proof of (96) and (97). Using the definition of $\check{\alpha}_{-}$(89), we proceed as on page 107 in [33] and we apply the bound (94) on $\left|\delta^{\prime}\right|$, so we get

$$
\left|\check{\alpha}_{-}^{\prime} \check{\alpha}_{-}-\check{\varphi}_{\delta}\left(\check{q}_{-}, \partial_{s} \check{q}_{-}\right)\right| \leq C_{0}\left|\delta^{\prime}\right| \frac{\check{\alpha}_{-}^{2}}{1-\delta^{2}} \leq C_{0}\left(\check{\alpha}_{1}^{2}+\check{\alpha}_{-}^{2}+\tilde{\alpha}_{-}^{2}\right)^{2}
$$


Using Lemma 4.4, we write

$$
\begin{aligned}
& \left|\check{\alpha}_{-}^{\prime} \check{\alpha}_{-}-\check{\varphi}_{\delta}\left(\check{q}_{-}, \check{L}_{\delta} \check{q}_{-}\right)-\int_{-1}^{1} \check{q}_{2} \check{f}_{\delta}\left(q_{1}\right) \rho d y\right| \\
& \leq C_{0}\left(\check{\alpha}_{1}^{2}+\check{\alpha}_{-}^{2}+\tilde{\alpha}_{-}^{2}\right)^{\frac{3}{2}}+\left|\check{\varphi}_{\delta}\left(\check{q}_{-}, \partial_{s} \check{q}_{-}-\check{L}_{\delta}\left(\check{q}_{-}\right)-\check{\pi}_{-}^{\delta}\left(\begin{array}{c}
0 \\
\check{f}_{\delta}\left(q_{1}\right)
\end{array}\right)\right)\right| \\
& \leq C_{0}\left(\check{\alpha}_{1}^{2}+\check{\alpha}_{-}^{2}+\tilde{\alpha}_{-}^{2}\right)^{\frac{3}{2}}+|| \check{q}_{-}||{ }_{\mathcal{H}}\left(\check{\alpha}_{1}^{2}+\check{\alpha}_{-}^{2}+\tilde{\alpha}_{-}^{2}\right)^{\frac{3}{2}} \leq C_{0}\left(\check{\alpha}_{1}^{2}+\check{\alpha}_{-}^{2}+\tilde{\alpha}_{-}^{2}\right)^{\frac{3}{2}} .
\end{aligned}
$$

Since we easily get from the definition (32) of $\check{L}_{\delta}$ that

$$
\check{\varphi}_{\delta}\left(\check{q}_{1} \check{L}_{\delta}(\check{q})\right)=-\frac{4}{p-1} \int_{-1}^{1} \check{q}_{-, 2}^{2} \frac{\rho}{1-y^{2}} d y
$$

we conclude that

$$
\left|\check{\alpha}_{-}^{\prime} \check{\alpha}_{-}-\int_{-1}^{1} \check{q}_{2} \check{f}_{\delta}\left(q_{1}\right) \rho d y\right| \leq-\frac{4}{p-1} \int_{-1}^{1} \check{q}_{-, 2}^{2} \frac{\rho}{1-y^{2}} d y+C_{0}\left(\check{\alpha}_{1}^{2}+\check{\alpha}_{-}^{2}+\tilde{\alpha}_{-}^{2}\right)^{\frac{3}{2}} .
$$

Arguing similarly for $\tilde{\alpha}_{-}$(89), we see that

$$
\tilde{\alpha}_{-}^{2}(s)=\tilde{\varphi}_{\delta}(\tilde{q}(s), \tilde{q}(s)) .
$$

Using the definition (58) of $\tilde{\varphi}_{\delta}$, we have by differentiation

$$
\tilde{\alpha}_{-}^{\prime} \tilde{\alpha}_{-}=\tilde{\varphi}_{\delta}\left(\tilde{q}, \partial_{s} \tilde{q}\right)-\frac{1}{2} \delta^{\prime}(s) \int_{-1}^{1} \partial_{\delta} \tilde{\psi}(\delta, y) \tilde{q}_{1}^{2} \rho .
$$

Using the Hölder inequality, the Hardy-Sobolev estimate of Lemma C.2 and (91), we write

$$
\left|\int_{-1}^{1} \partial_{\delta} \tilde{\psi}(\delta, y) \tilde{q}_{1}^{2} \rho\right| \leq\left\|\partial_{\delta} \tilde{\psi}(\delta, y)\right\|_{L_{\rho}^{\frac{p+1}{p-1}}}\left\|\tilde{q}_{1}\right\|_{L_{\rho}^{p+1}}^{2} \leq C_{0}\left\|\partial_{\delta} \tilde{\psi}(\delta, y)\right\|_{L_{\rho}^{\frac{p+1}{p-1}}} \tilde{\alpha}_{-}(s)^{2} .
$$

Since $\left|\partial_{\delta} \tilde{\psi}(\delta, y)\right| \leq C /(1+\delta y)^{2}$ for all $(\delta, y) \in(-1,1)^{2}$ from the expression of $\tilde{\psi}$ in (30), using Claim 3.2, we see that $\left\|\partial_{\delta} \tilde{\psi}(\delta, y)\right\|_{L_{\rho}^{p-1}}^{\frac{p+1}{p-1}} \leq C /\left(1-\delta^{2}\right)$. Therefore, using (116), (117), and the bound (94) on $\left|\delta^{\prime}(s)\right|$, we get

$$
\left|\tilde{\alpha}_{-}^{\prime} \tilde{\alpha}_{-}-\tilde{\varphi}_{\delta}\left(\tilde{q}, \partial_{s} \tilde{q}\right)\right| \leq C_{0}\left|\delta^{\prime}\right| \frac{\tilde{\alpha}_{-}^{2}}{1-\delta^{2}} \leq C_{0}\left(\check{\alpha}_{1}^{2}+\check{\alpha}_{-}^{2}+\tilde{\alpha}_{-}^{2}\right)^{2}
$$

From (118), the continuity of $\tilde{\varphi}_{\delta}$, and Lemma 4.5, we write

$$
\begin{aligned}
& \left|\tilde{\alpha}_{-}^{\prime} \tilde{\alpha}_{-}-\tilde{\varphi}_{\delta}\left(\tilde{q}, \tilde{L}_{\delta} \tilde{q}\right)-\int_{-1}^{1} \tilde{q}_{2} \tilde{f}_{\delta}\left(q_{1}\right) \rho d y\right| \\
& \leq C_{0}\left(\check{\alpha}_{1}^{2}+\check{\alpha}_{-}^{2}+\tilde{\alpha}_{-}^{2}\right)^{\frac{3}{2}}+\left|\tilde{\varphi}_{\delta}\left(\tilde{q}, \partial_{s} \tilde{q}-\tilde{L}_{\delta}(\tilde{q})-\tilde{\pi}_{-}^{\delta}\left(\begin{array}{c}
0 \\
\tilde{f}_{\delta}\left(q_{1}\right)
\end{array}\right)\right)\right| \\
& \leq C_{0}\left(\check{\alpha}_{1}^{2}+\check{\alpha}_{-}^{2}+\tilde{\alpha}_{-}^{2}\right)^{\frac{3}{2}}+\|\tilde{q}\|_{\mathcal{H}}\left(\check{\alpha}_{1}^{2}+\check{\alpha}_{-}^{2}+\tilde{\alpha}_{-}^{2}\right)^{\frac{3}{2}} \leq C_{0}\left(\check{\alpha}_{1}^{2}+\check{\alpha}_{-}^{2}+\tilde{\alpha}_{-}^{2}\right)^{\frac{3}{2}} .
\end{aligned}
$$


Besides, using the expressions of $\tilde{L}_{\delta}$ (34) and $\tilde{\varphi}_{\delta}$ (158), we have

$$
\begin{aligned}
& \tilde{\varphi}_{\delta}\left(\tilde{q}, \tilde{L}_{\delta}(\tilde{q})\right)=\tilde{\varphi}_{\delta}\left(\left(\mathcal{L} \tilde{q}_{1}+\tilde{\psi}(\delta, y) \tilde{q}_{1}-\frac{p+3}{p-1} \tilde{q}_{2}-2 y \partial_{y} \tilde{q}_{2}\right),\left(\begin{array}{c}
\tilde{q}_{1} \\
\tilde{q}_{2}
\end{array}\right)\right) \\
& =-\int_{-1}^{1} \tilde{q}_{2}\left(\mathcal{L} \tilde{q}_{1}+\tilde{\psi}(\delta, y) \tilde{q}_{1}\right) \rho d y \\
& +\int_{-1}^{1}\left(\mathcal{L} \tilde{q}_{1}+\tilde{\psi}(\delta, y) \tilde{q}_{1}-\frac{p+3}{p-1} \tilde{q}_{2}-2 y \tilde{q}_{2}^{\prime}\right) \tilde{q}_{2} \rho d y \\
& =-\frac{p+3}{p-1} \int_{-1}^{1} \tilde{q}_{2}^{2} \rho d y-\int_{-1}^{1} y\left(\tilde{q}_{2}^{2}\right)^{\prime} \rho d y=-\frac{p+3}{p-1} \int_{-1}^{1} \tilde{q}_{2}^{2}+\int_{-1}^{1} \tilde{q}_{2}^{2}\left(\rho-y \rho^{\prime}\right) d y \\
(120) & =-\frac{4}{p-1}\left[\int_{-1}^{1} \tilde{q}_{-, 2}^{2} \rho d y+\int_{-1}^{1} \tilde{q}_{-, 2}^{2} \frac{y^{2} \rho}{1-y^{2}} d y\right]=-\frac{4}{p-1} \int_{-1}^{1} \tilde{q}_{-, 2}^{2} \frac{\rho}{1-y^{2}} d y .
\end{aligned}
$$

Using (119) and (120), we see that

$$
\left|\tilde{\alpha}_{-}^{\prime} \tilde{\alpha}_{-}-\int_{-1}^{1} \tilde{q}_{2} \tilde{f}_{\delta}\left(q_{1}\right) \rho d y\right| \leq-\frac{4}{p-1} \int_{-1}^{1} \tilde{q}_{-, 2}^{2} \frac{\rho}{1-y^{2}} d y+C_{0}\left(\check{\alpha}_{1}^{2}+\check{\alpha}_{-}^{2}+\tilde{\alpha}_{-}^{2}\right)^{\frac{3}{2}} .
$$

Therefore, using (115) with (121), we write

$$
\begin{aligned}
& \left|\frac{1}{2}\left(\check{\alpha}_{-}^{2}+\tilde{\alpha}_{-}^{2}\right)^{\prime}-\left[\int_{-1}^{1} \check{q}_{2} \check{f}_{\delta}\left(q_{1}\right)+\tilde{q}_{2} \tilde{f}_{\delta}\left(q_{1}\right) \rho d y\right]\right| \\
& \leq-\frac{4}{p-1} \int_{-1}^{1}\left(\check{q}_{-, 2}^{2}+\tilde{q}_{-, 2}^{2}\right) \frac{\rho}{1-y^{2}} d y+C_{0}\left(\check{\alpha}_{1}^{2}+\check{\alpha}_{-}^{2}+\tilde{\alpha}_{-}^{2}\right)^{\frac{3}{2}}
\end{aligned}
$$

Better yet, by (114) we see that estimate (96) holds with $R_{-}$given by (92). Using Claim 4.3. Lemma C.2 and condition (86) (considering first the case $p \geq 2$ and then the case $1<p<2$ ), we see that (97) holds. It remains to prove Lemma 4.5 in order to conclude the proof of (98) and (100).

\section{Proof of Lemma 4.5 .}

- Proof of (112): We first project equation (75) using the negative projector $\tilde{\pi}_{-}^{\delta}$ introduced in Definition 3.6

$$
\tilde{\pi}_{-}^{\delta}\left(\partial_{s} \tilde{q}\right)=\tilde{\pi}_{-}^{\delta}\left(\tilde{L}_{\delta(s)} \tilde{q}\right)+\tilde{\pi}_{-}^{\delta}\left(\begin{array}{c}
0 \\
\tilde{f}_{\delta}\left(q_{1}\right)
\end{array}\right)-\theta^{\prime}(s) \tilde{\pi}_{-}^{\delta}\left(\begin{array}{c}
\kappa(\delta, \cdot)+\check{q_{1}} \\
\check{q_{2}}
\end{array}\right) .
$$

We write the expansion (56) with $\partial_{s} \tilde{q}$

$$
\partial_{s} \tilde{q}=\tilde{\pi}_{0}^{\delta}\left(\partial_{s} \tilde{q}\right) \tilde{F}_{0}(\delta, \cdot)+\tilde{\pi}_{-}^{\delta}\left(\partial_{s} \tilde{q}\right) .
$$

Using (72) and (124), we see that

$$
\tilde{\pi}_{-}^{\delta}\left(\partial_{s} \tilde{q}\right)-\partial_{s} \tilde{q}=0 .
$$

From the remark after Definition [3.6, we see that $\tilde{L}_{\delta}(\tilde{q}) \in \mathcal{H}_{-}\left(\right.$as $\left.\tilde{q} \in \mathcal{H}_{-}\right)$and

$$
\tilde{\pi}_{-}^{\delta}\left(\tilde{L}_{\delta}(\tilde{q})\right)=\tilde{L}_{\delta}(\tilde{q}) .
$$

Using (56) with $(\kappa(\delta, y), 0)$, (49) and (55), we get

$$
\tilde{\pi}_{-}^{\delta}\left(\begin{array}{c}
\kappa(\delta, \cdot) \\
0
\end{array}\right)=0 ;
$$


therefore, using (56) with $\check{q}$, we write

$$
\begin{aligned}
\left\|\tilde{\pi}_{-}^{\delta}\left(\begin{array}{c}
\kappa(\delta, y)+\check{q_{1}} \\
\check{q_{2}}
\end{array}\right)\right\|_{\mathcal{H}} & =\left\|\tilde{\pi}_{-}^{\delta}\left(\begin{array}{c}
\check{\check{q}_{1}} \\
\check{q_{2}}
\end{array}\right)\right\|_{\mathcal{H}} \leq\|\check{q}\|_{\mathcal{H}}+\left|\tilde{\pi}_{0}^{\delta}(\check{q})\right|\left\|\tilde{F}_{0}(\delta, \cdot)\right\|_{\mathcal{H}} \\
& \leq C\|\check{q}\|_{\mathcal{H}} \leq C_{0}\left(\check{\alpha}_{1}^{2}+\check{\alpha}_{-}^{2}+\tilde{\alpha}_{-}^{2}\right)^{\frac{1}{2}}
\end{aligned}
$$

where we used (55), (50), (43) and (91) to get the last line. Using (123), (125), (126), (127) and (94) we get (112).

- Proof of (113): Note from (56) that

$$
\left(\begin{array}{c}
0 \\
\tilde{f}_{\delta}\left(q_{1}\right)
\end{array}\right)=\tilde{B}_{0}(s) \tilde{F}_{0}(\delta, \cdot)+\tilde{\pi}_{-}^{\delta}\left(\begin{array}{c}
0 \\
\tilde{f}_{\delta}\left(q_{1}\right)
\end{array}\right),
$$

where $\tilde{B}_{0}(s)=\tilde{\pi}_{0}^{\delta}\left(\begin{array}{c}0 \\ \tilde{f}_{\delta}\left(q_{1}\right)\end{array}\right)$. So from the definition (58), the bilinearity of $\tilde{\varphi}_{\delta}$ and the bound on the norm of $\tilde{F}_{0}$, we have

$$
\begin{aligned}
& \left|\tilde{\varphi}_{\delta}\left(\tilde{q}, \tilde{\pi}_{-}^{\delta}\left(\begin{array}{c}
0 \\
\tilde{f}_{\delta}\left(q_{1}\right)
\end{array}\right)\right)-\int_{-1}^{1} \tilde{q}_{2} \tilde{f}_{\delta}\left(q_{1}\right) \rho d y\right| \\
= & \left|\tilde{\varphi}_{\delta}\left(\tilde{q},\left(\begin{array}{c}
0 \\
\tilde{f}_{\delta}\left(q_{1}\right)
\end{array}\right)-\tilde{B}_{0}(s) \tilde{F}_{0}(\delta, y)\right)-\tilde{\varphi}_{\delta}\left(\tilde{q},\left(\begin{array}{c}
0 \\
\tilde{f}_{\delta}\left(q_{1}\right)
\end{array}\right)\right)\right| \\
= & \left|\tilde{\varphi}_{\delta}\left(\tilde{q}, \tilde{B}_{0}(s) \tilde{F}_{0}(\delta, y)\right)\right| \leq C\left|\tilde{B}_{0}(s)\right||| \tilde{q} \|_{\mathcal{H}} \leq C\left(\check{\alpha}_{1}^{2}+\check{\alpha}_{-}^{2}+\tilde{\alpha}_{-}^{2}\right)^{\frac{3}{2}} .
\end{aligned}
$$

From (108) and (91), (113) follows.

[• Proof of (114)] We see from the expression of $\mathcal{F}_{\delta(s)}\left(q_{1}\right)$ (93) that

$$
\begin{aligned}
& \int_{-1}^{1} \check{q}_{2} \check{f}_{\delta}\left(q_{1}\right)+\int_{-1}^{1} \tilde{q}_{2} \tilde{f}_{\delta}\left(q_{1}\right) \rho d y=\int_{-1}^{1} \partial_{s} \mathcal{F}_{\delta(s)}\left(q_{1}\right) \rho d y \\
& +\int_{-1}^{1} \frac{p-1}{2} \kappa^{p-2}(\delta) \partial_{\delta} \kappa(\delta) \delta^{\prime}(s)\left(p \check{q}_{1}^{2}+\tilde{q}_{1}^{2}\right) \rho d y \\
& +\theta^{\prime}(s) \int_{-1}^{1}\left[\left(\kappa(\delta, y)+\check{q}_{1}\right) \tilde{f}_{\delta}\left(q_{1}\right)-\tilde{q}_{1} \check{f}_{\delta}\left(q_{1}\right)\right] \rho d y .
\end{aligned}
$$

Since we have $\left\|\partial_{\delta} \kappa(\delta, \cdot) \kappa(\delta, .)^{p-2}\right\|_{L_{\rho}^{\frac{p+1}{p-1}}} \leq C_{0} /\left(1-\delta^{2}\right)$, from the expression of $\partial_{\delta} \kappa(\delta, y)$,

$$
\partial_{\delta} \kappa(\delta, y)=-\frac{2 \kappa_{0}}{p-1} \frac{(y+\delta)\left(1-\delta^{2}\right)^{\frac{2-p}{p-1}}}{(1+\delta y)^{-\frac{p+1}{p-1}}},
$$

the definition of (10) $\kappa(\delta, y)$ and Claim 3.2, we use the Hölder inequality and the Hardy-Sobolev inequality of Lemma C.2 to derive that

$$
\left|\int_{-1}^{1} \partial_{\delta} \kappa(\delta, y) \kappa(\delta, y)^{p-2}\left(p \check{q}_{1}^{2}+\tilde{q}_{1}^{2}\right) \rho d y\right| \leq \frac{C_{0}}{1-\delta^{2}}\|q(s)\|_{\mathcal{H}}^{2},
$$

so, by (91) and (94), we get

$$
\left|(p-1) \delta^{\prime}(s) \int_{-1}^{1} \kappa^{p-1}(\delta, y) \partial_{\delta} \kappa(\delta, y)\left(p \check{q}_{1}^{2}+\tilde{q}_{1}^{2}\right) \rho d y\right| \leq C_{0}\left(\check{\alpha}_{1}^{2}+\check{\alpha}_{-}^{2}+\tilde{\alpha}_{-}^{2}\right)^{2} .
$$


Since $\forall|\delta|<1,\|\kappa(\delta, \cdot)\|_{\mathcal{H}_{0}} \leq C_{0}$ by definition (10), using Claim 4.3, Lemma C.2 and (91) we see that

$$
\begin{aligned}
& \left|\int_{-1}^{1} \check{q}_{1} \tilde{f}_{\delta}\left(q_{1}\right) \rho(y) d y\right|+\left|\int_{-1}^{1} \tilde{q}_{1} \check{f}_{\delta}\left(q_{1}\right) \rho(y) d y\right| \\
& \leq C_{0}\left(\delta_{\{p \geq 2\}} \int_{-1}^{1} \kappa(\delta, y)^{p-2}\left|q_{1}(y, s)\right|^{3} \rho d y+\int_{-1}^{1}\left|q_{1}(y, s)\right|^{p+1} \rho d y\right) \\
& \leq C_{0}\left(\delta_{\{p \geq 2\}}\|\kappa(\delta, \cdot)\|_{L_{\rho}^{p+1}}^{p-2}\left\|q_{1}\right\|_{L_{\rho}^{p+1}}^{p+1}+\left\|q_{1}\right\|_{L_{\rho}^{p+1}}^{p+1}\right) \\
& \leq C_{0}\left\|q_{1}(s)\right\|_{\mathcal{H}}^{\check{p}+1}, \quad \text { where } \check{p}=\min (p, 2)>1 \\
& \leq C_{0}\left(\check{\alpha}_{1}^{2}+\check{\alpha}_{-}^{2}+\tilde{\alpha}_{-}^{2}\right) .
\end{aligned}
$$

Using (128), (129), (108), (130) and (94) we see that estimate (114) holds. This concludes the proof of Lemma 4.5 .

Part 3 : An additional relation. (iii) First, note from (91) that

$$
\begin{aligned}
& \int_{-1}^{1} \check{q}_{1}^{2} \rho+\int_{-1}^{1}\left(\partial_{y} \check{q}_{1}\right)^{2}\left(1-y^{2}\right) \rho+\int_{-1}^{1} \check{q}_{2}^{2} \rho \leq C_{0}\left(\check{\alpha}_{1}^{2}+\check{\alpha}_{-}^{2}\right) \\
& \int_{-1}^{1} \tilde{q}_{1}^{2} \rho+\int_{-1}^{1}\left(\partial_{y} \tilde{q}_{1}\right)^{2}\left(1-y^{2}\right) \rho+\int_{-1}^{1} \tilde{q}_{2}^{2} \rho \leq C_{0} \tilde{\alpha}_{-}^{2}
\end{aligned}
$$

and, using equation (74) and the definition of $\check{L}_{\delta}$ (32), we write

$$
\begin{aligned}
& \frac{d}{d s} \int_{-1}^{1} \check{q}_{1} \check{q}_{2} \rho=\int_{-1}^{1}\left(\theta^{\prime}(s) \tilde{q}_{1} \check{q}_{2}+\check{q}_{2}^{2}-\delta^{\prime}(s) \check{q}_{2} \partial_{\delta} \kappa(\delta, \cdot)\right) \rho \\
+ & \int_{-1}^{1} \check{q}_{1}\left(\mathcal{L} \check{q_{1}}+\check{\psi}(\delta, \cdot) \check{q_{1}}-\frac{p+3}{p-1} \check{q_{2}}-2 y \partial_{y} \check{q_{2}}+\check{f}_{\delta}\left(q_{1}\right)+\theta^{\prime}(s) \tilde{q}_{2}\right) \rho .
\end{aligned}
$$

Almost all of the terms in the right hand side of (133) have been studied in 33 , except for the two terms with $\theta^{\prime}(s)$.

Using the Cauchy-Schwartz inequality, (86), (91) and (94), we see that

$$
\left|\int_{-1}^{1} \theta^{\prime}(s) \tilde{q}_{1} \check{q}_{2} \rho\right| \leq\left|\theta^{\prime}(s)\right|\left(\int_{-1}^{1} \tilde{q}_{1}^{2} \rho\right)^{\frac{1}{2}}\left(\int_{-1}^{1} \check{q}_{2}^{2} \rho\right)^{\frac{1}{2}} \leq \frac{1}{100}\left(\check{\alpha}_{1}^{2}+\check{\alpha}_{-}^{2}+\tilde{\alpha}_{-}^{2}\right),
$$

and

$$
\left|\int_{-1}^{1} \theta^{\prime}(s) \check{q}_{1} \tilde{q}_{2} \rho\right| \leq\left|\theta^{\prime}(s)\right|\left(\int_{-1}^{1} \check{q}_{1}^{2} \rho\right)^{\frac{1}{2}}\left(\int_{-1}^{1} \tilde{q}_{2}^{2} \rho\right)^{\frac{1}{2}} \leq \frac{1}{100}\left(\check{\alpha}_{1}^{2}+\check{\alpha}_{-}^{2}+\tilde{\alpha}_{-}^{2}\right),
$$

for $\epsilon$ small enough. Using the proof of Proposition 5.2 on page 103 in 33 ] to control the other terms we get (98).

In the same way, in order to prove (99), we will bound all the terms on the right hand side of the following:

$$
\begin{gathered}
\frac{d}{d s} \int_{-1}^{1} \tilde{q}_{1} \tilde{q}_{2} \rho=\int_{-1}^{1}\left(-\theta^{\prime}(s) \tilde{q}_{2}\left(\kappa(\delta, \cdot)+\tilde{q}_{1}\right)+\tilde{q}_{2}^{2}\right) \rho \\
+\quad \int_{-1}^{1} \tilde{q}_{1}\left(\mathcal{L} \tilde{q_{1}}+\tilde{\psi}(\delta, \cdot) \tilde{q_{1}}-\frac{p+3}{p-1} \tilde{q_{2}}-2 y \partial_{y} \tilde{q_{2}}+\tilde{f}_{\delta}\left(q_{1}\right)-\theta^{\prime}(s) \check{q}_{2}\right) \rho .
\end{gathered}
$$


We use the Cauchy-Schwartz inequality, (94), (131), (132), (86) and bound $\kappa(\delta, y)$ to write for $\epsilon$ small enough,

$$
\begin{aligned}
\left|\int_{-1}^{1} \theta^{\prime}(s) \tilde{q}_{2}\left(\kappa(\delta, y)+\tilde{q}_{1}\right) \rho\right| & \leq\left|\theta^{\prime}(s)\right|\left(\|\kappa(\delta, \cdot)\|_{L_{\rho}^{2}}+\left(\int_{-1}^{1} \tilde{q}_{1}^{2} \rho\right)^{\frac{1}{2}}\right)\left(\int_{-1}^{1} \tilde{q}_{2}^{2} \rho\right)^{\frac{1}{2}} \\
& \leq C\left(\check{\alpha}_{1}^{2}+\check{\alpha}_{-}^{2}+\tilde{\alpha}_{-}^{2}\right)^{\frac{3}{2}} \leq \frac{1}{100}\left(\check{\alpha}_{1}^{2}+\check{\alpha}_{-}^{2}+\tilde{\alpha}_{-}^{2}\right) .
\end{aligned}
$$

From the definition of $\tilde{\varphi}_{\delta}$ (58) and the definition of $\tilde{\alpha}_{-}$(90), we write

$$
\int_{-1}^{1} \tilde{q}_{1}\left(\mathcal{L} \tilde{q_{1}}+\tilde{\psi}(\delta, \cdot) \tilde{q_{1}}\right) \rho=-\tilde{\varphi}_{\delta}\left(\left(\begin{array}{c}
\tilde{q_{1}} \\
0
\end{array}\right),\left(\begin{array}{c}
\tilde{q_{1}} \\
0
\end{array}\right)\right)=-\tilde{\alpha}_{-}^{2} .
$$

Using integration by parts, the fact that $\left|y \partial_{y} \rho(y)\right| \leq C \frac{\rho}{1-y^{2}}$, the Cauchy-Schwartz inequality, Lemma C.2 (131) and (132), we write

$$
\begin{aligned}
& \left|-\frac{p+3}{p-1} \int_{-1}^{1} \tilde{q}_{1} \tilde{q}_{1} \rho-2 \int_{-1}^{1} \tilde{q}_{1} y \partial_{y} \tilde{q}_{2} \rho\right| \\
= & \left|2 \int_{-1}^{1} \tilde{q}_{2} \partial_{y} \tilde{q}_{1} y \rho+\left(2-\frac{p+3}{p-1}\right) \int_{-1}^{1} \tilde{q}_{1} \tilde{q}_{1} \rho+2 \int_{-1}^{1} \tilde{q}_{2} \tilde{q}_{1} y \partial_{y} \rho\right| \\
\leq & C \int_{-1}^{1}\left(\left|\tilde{q}_{2}\right|\left|\partial_{y} \tilde{q}_{1}\right| \rho+\left|\tilde{q}_{2}\right|\left|\tilde{q}_{1}\right| \frac{\rho}{1-y^{2}}\right) \\
\leq & \left(\int_{-1}^{1} \tilde{q}_{2}^{2} \frac{\rho}{1-y^{2}}\right)^{1 / 2}\left[\int_{-1}^{1}\left(\partial_{y} \tilde{q}_{1}\right)^{2}\left(1-y^{2}\right) \rho+\int_{-1}^{1} \tilde{q}_{1}^{2} \frac{\rho}{1-y^{2}}\right]^{1 / 2} \\
\leq & C_{0} \tilde{\alpha}_{-}\left(\int_{-1}^{1} \tilde{q}_{2}^{2} \frac{\rho}{1-y^{2}}\right)^{1 / 2} \leq \frac{1}{100} \tilde{\alpha}_{-}^{2}+C \int_{-1}^{1} \tilde{q}_{2}^{2} \frac{\rho}{1-y^{2}} .
\end{aligned}
$$

Arguing as for (130) and (134) and using (86) we write for $\epsilon$ small enough

$$
\begin{aligned}
\left|\int_{-1}^{1} \tilde{q}_{1} \tilde{f}_{\delta}\left(q_{1}\right) \rho(y) d y\right| & \leq C_{0}|| q_{1}(s)||_{\mathcal{H}}^{\check{\check{H}+1}} \leq \frac{1}{100}\left(\check{\alpha}_{1}^{2}+\check{\alpha}_{-}^{2}+\tilde{\alpha}_{-}^{2}\right), \\
\left|\int_{-1}^{1} \theta^{\prime}(s) \check{q}_{2} \tilde{q}_{1} \rho\right| & \leq C\left|\theta^{\prime}(s)\right|\left(\check{\alpha}_{1}^{2}+\check{\alpha}_{-}^{2}+\tilde{\alpha}_{-}^{2}\right) \leq \frac{1}{100}\left(\check{\alpha}_{1}^{2}+\check{\alpha}_{-}^{2}+\tilde{\alpha}_{-}^{2}\right) .
\end{aligned}
$$

Collecting (136)-(141), we get

$$
\frac{d}{d s} \int_{-1}^{1} \tilde{q}_{1} \tilde{q}_{2} \rho \leq-\frac{4}{5} \tilde{\alpha}_{-}^{2}+C_{0} \int_{-1}^{1} \tilde{q}_{2}^{2} \frac{\rho}{1-y^{2}}+C_{0}\left(\check{\alpha}_{1}^{2}+\check{\alpha}_{-}^{2}\right) .
$$

Part 4 : Energy barrier. (iv) Using the definition of $q(y, s)(73)$, we can make an expansion of $E\left(w(s), \partial_{s} w(s)\right)$ (9) for $q \rightarrow 0$ in $\mathcal{H}$ and obtain from straightforward computations:

$$
E\left(w(s), \partial_{s} w(s)\right)=E\left(\kappa_{0}, 0\right)+\frac{1}{2}\left(\check{\varphi}_{\delta}(\check{q}, \check{q})+\tilde{\varphi}_{\delta}(\tilde{q}, \tilde{q})\right)-\int_{-1}^{1} \mathcal{F}_{\delta}\left(q_{1}\right) \rho d y,
$$

where $\check{\varphi}_{\delta}, \tilde{\varphi}_{\delta}$ and $\mathcal{F}_{\delta}\left(q_{1}\right)$ are defined in (40), (58) and (93).

Since we have (92), (97), (86) and (91):

$$
\left|\int_{-1}^{1} \mathcal{F}_{\delta}\left(q_{1}\right) \rho d y\right| \leq C\|q(s)\|_{\mathcal{H}}^{\check{p}+1} \leq C \epsilon^{\check{p}-1}\left(\check{\alpha}_{1}^{2}+\check{\alpha}_{-}^{2}+\tilde{\alpha}_{-}^{2}\right)
$$

we note that for some $C_{1}>0$

$$
\check{\varphi}_{\delta}(\check{q}, \check{q}) \leq C_{0} \check{\alpha}_{1}^{2}-C_{1} \check{\alpha}_{-}^{2},
$$


which was proved in [33] on page 113. From (12), (142), (144) and (143), we see that taking $\epsilon$ small enough so that $C \epsilon^{\check{p}-1}<\frac{C_{1}}{4}$, we get

$$
0 \leq E\left(w(s), \partial_{s} w(s)\right)-E\left(\kappa_{0}, 0\right) \leq\left(\frac{C_{0}}{2}+\frac{C_{1}}{4}\right) \check{\alpha}_{-}^{2}-\frac{C_{1}}{4} \check{\alpha}_{1}^{2}+\left(\frac{1}{2}+\frac{C_{1}}{4}\right) \tilde{\alpha}_{-}^{2},
$$

which yields (100).

4.4. Exponential decay of the different components. Our aim is to show that $\|q(s)\|_{\mathcal{H}} \rightarrow 0$ and that both $\theta$ and $\delta$ converge as $s \rightarrow \infty$. An important issue will be to show that the unstable mode $\check{\alpha}_{1}$, which satisfies equation (91), never dominates. This is true thanks to item (iv) in Proposition 4.2 (see the third remark following that Proposition for more details). Let us first introduce a more adapted notation and rewrite Proposition 4.2.

If we introduce

$$
\lambda(s)=\frac{1}{2} \log \left(\frac{1+\delta(s)}{1-\delta(s)}\right), a(s)=\check{\alpha}_{1}(s)^{2} \text { and } b(s)=\check{\alpha}_{-}(s)^{2}+\tilde{\alpha}_{-}(s)^{2}+R_{-}(s)
$$

(note that $\delta(s)=\tanh (\lambda(s))$ ), then we see from (97) and (91) that if (86) holds, then $\left|b-\left(\check{\alpha}_{-}(s)^{2}+\tilde{\alpha}_{-}(s)^{2}\right)\right| \leq C_{0} \epsilon^{\check{p}-1}\left(\check{\alpha}_{1}(s)^{2}+\check{\alpha}_{-}(s)^{2}+\tilde{\alpha}_{-}(s)^{2}\right)$. Hence

$$
\frac{99}{100} \check{\alpha}_{-}(s)^{2}+\frac{99}{100} \tilde{\alpha}_{-}(s)^{2}-\frac{1}{100} a \leq b \leq \frac{101}{100} \check{\alpha}_{-}(s)^{2}+\frac{101}{100} \tilde{\alpha}_{-}(s)^{2}+\frac{1}{100} a
$$

for $\epsilon$ small enough. Therefore, using Proposition 4.2, estimate (86), (91) and the fact that $\lambda^{\prime}(s)=\frac{\delta^{\prime}(s)}{1-\delta(s)^{2}}$, we derive the following:

Claim 4.6 (Relations between $a, b, \lambda, \theta, \int_{-1}^{1} \check{q}_{1} \check{q}_{2} \rho$ and $\left.\int_{-1}^{1} \tilde{q}_{1} \tilde{q}_{2} \rho\right)$. There exist positive $\epsilon_{4}, K_{4}$ and $K_{5}$ such that if $w$ is a solution to equation (5) such that (72) and (86) hold at some time $s$ for some $\epsilon \leq \epsilon_{4}$, where $q$ is defined in (73), then using the notation (145), we have:

(i) (Size of the solution)

$$
\begin{aligned}
\frac{1}{K_{4}}(a(s)+b(s)) \leq\|q(s)\|_{\mathcal{H}}^{2} & \leq K_{4}(a(s)+b(s)) \leq K_{4}^{2} \epsilon^{2}, \\
\left|\theta^{\prime}(s)\right|+\left|\lambda^{\prime}(s)\right| & \leq K_{4}(a(s)+b(s)) \leq K_{4}^{2}\|q(s)\|_{\mathcal{H}}^{2}, \\
\left|\int_{-1}^{1} \check{q}_{1} \check{q}_{1} \rho\right| & \leq K_{4}(a(s)+b(s)), \\
\left|\int_{-1}^{1} \tilde{q}_{1} \tilde{q}_{1} \rho\right| & \leq K_{4} b(s),
\end{aligned}
$$

and (146) holds.

(ii) (Equations)

$$
\begin{aligned}
\frac{3}{2} a-K_{4} \epsilon b & \leq a^{\prime} \leq \frac{5}{2} a-K_{4} \epsilon b, \\
b^{\prime} & \leq-\frac{8}{p-1} \int_{-1}^{1}\left(\check{q}_{-, 2}^{2}+\tilde{q}_{-, 2}^{2}\right) \frac{\rho}{1-y^{2}} d y+K_{4} \epsilon(a+b), \\
\frac{d}{d s} \int_{-1}^{1}\left(\check{q}_{1} \check{q}_{2}+\tilde{q}_{1} \tilde{q}_{2}\right) \rho & \leq-\frac{3}{5} b+K_{4} \int_{-1}^{1}\left(\check{q}_{-, 2}^{2}+\tilde{q}_{2}^{2}\right) \frac{\rho}{1-y^{2}}+K_{4} a .
\end{aligned}
$$

(iii) (Energy barrier) If (12) holds, then

$$
a(s) \leq K_{5} b(s) \text {. }
$$


Proof of Theorem 4. Consider $w \in C\left(\left[s^{*}, \infty\right), \mathcal{H}\right)$ for some $s^{*} \in \mathbb{R}$ a solution of equation (5) such that (12) and (13) hold for some $\delta^{*} \in(-1,1), \theta^{*} \in[0,2 \pi)$ and $\epsilon^{*} \in\left(0, \epsilon_{0}\right]$. Consider then

$$
\epsilon=2 K_{0} K_{1} \epsilon^{*}
$$

where $K_{1}$ is given in Proposition 4.1 and $K_{0}$ will be fixed later. If

$$
\epsilon^{*} \leq \epsilon_{1} \text { and } \epsilon \leq \epsilon_{4},
$$

then we see that Proposition 4.1, Claim 4.6 and (146) apply respectively with $\epsilon^{*}$ and $\epsilon$. In particular, there is a maximal solution $\delta(s) \in C^{1}\left(\left[s^{*}, \infty\right),(-1,1)\right)$ such that (72) holds for all $s \in\left[s^{*}, \infty\right)$ where $q(y, s)$ is defined in (73) and

$$
\left|\theta-\theta^{*}\right|+\left|\lambda\left(s^{*}\right)-\lambda^{*}\right|+\left\|q\left(s^{*}\right)\right\|_{\mathcal{H}} \leq K_{1} \epsilon^{*} \text { with } \lambda^{*}=\log \left(\frac{1+\delta^{*}}{1-\delta^{*}}\right) .
$$

If in addition we have

$$
K_{0} \geq 1 \text {, hence } \epsilon \geq 2 K_{1} \epsilon^{*},
$$

then, we can give two definitions:

- We define first from (154) and (155) $s_{1}^{*} \in\left(s^{*}, \infty\right)$ such that for all $s \in\left[s^{*}, s_{1}^{*}\right]$,

$$
\|q(s)\|_{\mathcal{H}}<\epsilon
$$

and if $s_{1}^{*}<\infty$, then $\left\|q\left(s_{1}^{*}\right)\right\|_{\mathcal{H}}=\epsilon$.

-Then, we define $s_{2}^{*} \in\left[s^{*}, s_{1}^{*}\right]$ as the first $s \in\left[s^{*}, s_{1}^{*}\right]$ such that

$$
a(s) \geq \frac{b(s)}{5 K_{4}}
$$

where $K_{4}$ is introduced in Claim 4.6, or $s_{2}^{*}=s_{1}^{*}$ if (157) is never satisfied on $\left[s^{*}, s_{1}^{*}\right]$. We claim the following:

Claim 4.7. There exist positive $\epsilon_{6}, \mu_{6}, K_{6}$ and $f \in C^{1}\left(\left[s^{*}, s_{2}^{*}\right]\right.$ such that if $\epsilon \leq \epsilon_{6}$, then for all $s \in\left[s^{*}, s_{2}^{*}\right]$ :

(i)

$$
\frac{1}{2} f(s) \leq b(s) \leq 2 f(s) \text { and } f^{\prime}(s) \leq-2 \mu_{6} f(s)
$$

$$
\|q(s)\|_{\mathcal{H}} \leq K_{6}\left\|q\left(s^{*}\right)\right\|_{\mathcal{H}} e^{-\mu_{6}\left(s-s^{*}\right)} \leq K_{6} K_{1} \epsilon^{*} e^{-\mu_{6}\left(s-s^{*}\right)} .
$$

Proof. The proof of Claim 5.6 on page 115 in 33 remains valid where $f(s)$ is given by

$$
f(s)=b(s)+\eta_{6} \int_{-1}^{1}\left(\check{q}_{1} \check{q}_{2}+\tilde{q}_{1} \tilde{q}_{2}\right) \rho,
$$

where $\eta_{6}>0$ is fixed small independent of $\epsilon$.

Claim 4.8. (i) There exists $\epsilon_{7}>0$ such that for all $\sigma>0$, there exists $K_{7}(\sigma)>0$ such that if $\epsilon \leq \epsilon_{7}$, then

$$
\forall s \in\left[s_{2}^{*}, \min \left(s_{2}^{*}+\sigma, s_{1}^{*}\right)\right],\|q(s)\|_{\mathcal{H}} \leq K_{7}\left\|q\left(s^{*}\right)\right\|_{\mathcal{H}} e^{-\mu_{6}\left(s-s^{*}\right)} \leq K_{7} K_{1} \epsilon^{*} e^{-\mu_{6}\left(s-s^{*}\right)}
$$

where $\mu_{6}$ has been introduced in Claim 4.7 .

(ii) There exists $\epsilon_{8}>0$ such that if $\epsilon \leq \epsilon_{8}$, then

$$
\forall s \in\left(s_{2}^{*}, s_{1}^{*}\right], b(s) \leq a(s)\left(5 K_{4} e^{-\frac{\left(s-s_{2}^{*}\right)}{2}}+\frac{1}{4 K_{5}}\right)
$$

where $K_{4}$ and $K_{5}$ have been introduced in Claim 4.6 . 
Proof. The proof is the same as the proof of Claim 5.7 on page 117 in 33 .

Now, in order to conclude the proof of Theorem 4, we fix $\sigma_{0}>0$ such that

$$
5 K_{4}^{-\frac{\sigma_{0}}{2}}+\frac{1}{4 K_{5}} \leq \frac{1}{2 K_{5}}
$$

where $K_{4}$ and $K_{5}$ are introduced in Claim 4.6. Then, we impose the condition

$$
\epsilon=2 K_{0} K_{1} \epsilon^{*}, \text { where } K_{0}=\max \left(2, K_{6}, K_{7}\left(\sigma_{0}\right)\right),
$$

and the constants are defined in Proposition 4.1 and Claims 4.7 and 4.8 . Then, we fix

$$
\epsilon_{0}=\min \left(1, \epsilon_{1}, \frac{\epsilon_{i}}{2 K_{0} K_{1}} \text { for } i \in\{4,6,7,8\}\right)
$$

and the constants are defined in Proposition 4.1 and Claims 4.6, 4.7 and 4.8, Now, if $\epsilon^{*} \leq \epsilon_{0}$, then Claim 4.6. Claim 4.7 and Claim 4.8 apply. We claim that for all $s \in\left[s^{*}, s_{1}^{*}\right]$,

$$
\|q(s)\|_{\mathcal{H}} \leq K_{0}\left\|q\left(s^{*}\right)\right\|_{\mathcal{H}} e^{-\mu_{6}\left(s-s^{*}\right)} \leq K_{0} K_{1} \epsilon^{*} e^{-\mu_{6}\left(s-s^{*}\right)}=\frac{\epsilon}{2} e^{-\mu_{6}\left(s-s^{*}\right)} .
$$

Indeed, if $s \in\left[s^{*}, \min \left(s_{2}^{*}+\sigma_{0}, s_{1}^{*}\right)\right]$, then, this comes from (ii) of Claim 4.7 or $(i)$ of Claim 4.8 and the definition of $K_{0}$ (159).

Now, if $s_{2}^{*}+\sigma_{0}<s_{1}^{*}$ and $s \in\left[s_{2}^{*}+\sigma_{0}, s_{1}^{*}\right]$, then we have from (158) and the definition of $\sigma_{0}, b(s) \leq \frac{a(s)}{2 K_{5}}$ on the one hand. On the other hand, from (iii) in Claim 4.6. we have $a(s) \leq K_{5} b(s)$, hence $a(s)=b(s)=0$ and from (147), $q(y, s) \equiv 0$, hence (160) is satisfied trivially.

In particular, we have for all $s \in\left[s^{*}, s_{1}^{*}\right],\|q\|_{\mathcal{H}} \leq \frac{\epsilon}{2}$, hence, by definition of $s_{1}^{*}$, this means that $s_{1}^{*}=\infty$.

From $(i)$ of Claim 4.8 and (148), we have

$$
\forall s \geq s^{*},\|q(s)\|_{\mathcal{H}} \leq \frac{\epsilon}{2} e^{-\mu_{6}\left(s-s^{*}\right)} \text { and }\left|\theta^{\prime}(s)\right|+\left|\lambda^{\prime}(s)\right| \leq K_{4}^{2} \frac{\epsilon^{2}}{4} e^{-2 \mu_{6}\left(s-s^{*}\right)} .
$$

Hence, there is $\theta_{\infty}, \lambda_{\infty}$ in $\mathbb{R}$ such that $\theta(s) \rightarrow \theta_{\infty}, \lambda(s) \rightarrow \lambda_{\infty}$ as $s \rightarrow \infty$ and

$$
\begin{aligned}
& \forall s \geq s^{*},\left|\lambda_{\infty}-\lambda(s)\right| \leq C_{1} \epsilon^{* 2} e^{-2 \mu_{6}\left(s-s^{*}\right)}=C_{2} \epsilon^{2} e^{-2 \mu_{6}\left(s-s^{*}\right)}, \\
& \forall s \geq s^{*},\left|\theta_{\infty}-\theta(s)\right| \leq C_{1} \epsilon^{* 2} e^{-2 \mu_{6}\left(s-s^{*}\right)}=C_{2} \epsilon^{2} e^{-2 \mu_{6}\left(s-s^{*}\right)},
\end{aligned}
$$

for some positive $C_{1}$ and $C_{2}$. Taking $s=s^{*}$ here, and using (154) and (153), we see that $\left|\lambda_{\infty}-\lambda^{*}\right|+\left|\theta_{\infty}-\theta^{*}\right| \leq C_{0} \epsilon^{*}$. If $\delta_{\infty}=\tanh \lambda_{\infty}$, then we see that $\left|\delta_{\infty}-\delta^{*}\right| \leq C_{3}\left(1-\delta^{* 2}\right) \epsilon^{*}$.

Using the definition of $q$ (73), (79), (161), (162) and (163) we write

$$
\begin{aligned}
& \left\|\left(\begin{array}{c}
w(s) \\
\partial_{s} w(s)
\end{array}\right)-e^{i \theta_{\infty}}\left(\begin{array}{c}
\kappa\left(\delta_{\infty}, \cdot\right) \\
0
\end{array}\right)\right\|_{\mathcal{H}} \\
\leq & \left\|\left(\begin{array}{c}
w(s) \\
\partial_{s} w(s)
\end{array}\right)-e^{i \theta_{\infty}}\left(\begin{array}{c}
\kappa(\delta(s), \cdot) \\
0
\end{array}\right)\right\|_{\mathcal{H}}+\left\|e^{i \theta(s)}\left(\kappa(\delta(s), \cdot)-\kappa\left(\delta_{\infty}, \cdot\right)\right)\right\|_{\mathcal{H}_{0}} \\
+ & \left\|\kappa\left(\delta_{\infty}, \cdot\right)\right\|_{\mathcal{H}_{0}}\left|e^{i \theta(s)}-e^{i \theta(\infty)}\right| \\
\leq & \|q(s)\|_{\mathcal{H}}+C\left|\lambda_{\infty}-\lambda(s)\right|+C\left|\theta_{\infty}-\theta(s)\right| \leq C_{4} \epsilon^{*} e^{-\mu_{6}\left(s-s^{*}\right)} .
\end{aligned}
$$

This concludes the proof of Theorem 4 . 


\section{ApPendix A. EnERgy estimates in Similarity VARIABles}

For the sake of completeness, we give in this section sketches of the proofs of Propositions 1 and 3 proved in the real case in 32] and [33], and which extend to the complex case straightforwardly.

Sketch of the proof of Proposition 1, Consider $x_{0} \in \mathbb{R}$. If $T\left(x_{0}\right)=\min _{x \in \mathbb{R}} T(x)$, the proof is given in [30. If not, then we have to use a geometrical covering argument in addition to the ideas of [30. In order to keep this sketch to a reasonable length, we don't mention this covering argument and refer the reader to [32. Thus, we only focus on the real case where

$$
T_{0} \equiv T\left(x_{0}\right)=\min _{x \in \mathbb{R}} T(x) .
$$

- The lower bound:

Note first that the lower bound follows from the finite speed of propagation and scaling. Indeed, if $\left(w, \partial_{s} w\right)$ is small in $H^{1} \times L^{2}$ at some time $s=s_{0}$, then using back the similarity variables transformation (4), we see that initial data for $\left(u, \partial_{t} u\right)$ is small on the basis of the light cone, which means that the solution cannot blow-up at time $T$. See Remark after Theorem 1 on page 1149 in [30].

- The upper bound:

The proof is performed in similarity variables and relies on two arguments:

- The fact that the functional $E(w)$ defined in (9) is a Lyapunov functional for equation (5) which satisfies

$$
\frac{d}{d s} E(w(s))=-\frac{4}{p-1} \int_{-1}^{1}\left|\partial_{s} w\right|^{2} \frac{\rho}{1-y^{2}} d y .
$$

- A blow-up criterion from Antonini and Merle [3] stating that a solution $w$ of the equation cannot be defined for all $s \in\left[s_{0},+\infty\right)$ if $E\left(w\left(s_{0}\right)\right)<0$.

From these two facts, we see that for any $\bar{x} \in \mathbb{R}, w_{\bar{x}}$ satisfies

$$
\forall s \geq-\log T_{0},\left\{\begin{array}{l}
0 \leq E\left(w_{\bar{x}}(s)\right) \leq C_{0} \\
\int_{-\log T_{0}}^{+\infty} \int_{|y|<1}\left|\partial_{s} w\right|^{2} \frac{\rho}{1-y^{2}} \leq C_{0} .
\end{array}\right.
$$

With this identity, the proof is done in three steps:

Step 1. Multiplying (5) by $\bar{w} \rho$ and integrating for $x \in(-1,1)$, we obtain a new identity. Combining that identity with (164) we end up proving that

$$
\begin{aligned}
\forall \bar{x} \in \mathbb{R}, \forall s \leq & -\log T_{0}+1, \\
& \int_{s}^{s+1} \int_{|y|<\frac{1}{2}}\left(\left|\partial_{s} w_{\bar{x}}\right|^{2}+\left|\partial_{y} w_{\bar{x}}\right|^{2}\left(1-|y|^{2}\right)+\left|w_{\bar{x}}\right|^{p+1}\right) d y \leq C_{0} .
\end{aligned}
$$

Note that it is important to get (164) and (165) for any $\bar{x} \in \mathbb{R}$ and not just for $\bar{x}=x_{0}$.

Step 2. Using interpolation, Sobolev embeddings and a covering argument we end up with the fact that

$$
\forall \bar{x} \in \mathbb{R}, \forall s \geq-\log T_{0}+1, \int_{|y|<1}\left|w_{\bar{x}}\right|^{p+1} d y \leq C_{0} .
$$


Step 3. Given $s \geq-\log T_{0}+1$, we need to work at $\check{x}=\check{x}(s)$ such that

$$
\int_{|y|<1}\left|\nabla w_{\check{x}(s)}(y, s)\right|^{2} d y \geq \frac{1}{2} \sup _{x \in \mathbb{R}} \int_{|y|<1}\left|\nabla w_{x(s)}(y, s)\right|^{2} d y .
$$

Thanks to a covering technique, we see that at such an $\bar{x}(s)$, we have the equivalence of pure and weighted $L^{2}$ norm of the gradient, in the sense that

$$
\begin{aligned}
\frac{1}{C_{0}} \int_{|y|<1}\left|\nabla w_{\check{x}(s)}(y, s)\right|^{2} d y & \leq \int_{|y|<1}\left|\nabla w_{\check{x}(s)}(y, s)\right|^{2} \rho\left(1-y^{2}\right) d y \\
& \leq C_{0} \int_{|y|<1}\left|\nabla w_{\check{x}(s)}(y, s)\right|^{2} d y .
\end{aligned}
$$

This equivalence of norms is really crucial to finish the proof. Then we need the following and the Gagliardo-Nirenberg inequality (see Proposition 3.2 on page 1158 in 30]):

$$
\int_{|y|<1}|w|^{P+1} d y \leq C\left(\int_{|y|<1}\left(\partial_{y} w\right)^{2} d y\right)^{\beta} \text { for some } \beta<1 .
$$

Indeed, thanks to interpolation estimates in Sobolev spaces and the GagliardoNirenberg inequality (168), we use the energy boundedness (164) to derive that

$$
\forall s \geq-\log T_{0}+1, \int_{|y|<1}\left|\nabla w_{\check{x}(s)}(y, s)\right|^{2} d y \leq K\left(C_{0}\right)
$$

for some $K>0$. Using (167), we see that

$$
\forall s \geq-\log T_{0}+1, \forall \check{x} \in \mathbb{R}, \int_{|y|<1}\left|\nabla w_{\check{x}(s)}(y, s)\right|^{2} d y \leq 2 K\left(C_{0}\right) .
$$

Using the definition of the functional $E(w)$ (9) and a covering argument together with (166), we conclude the proof of the upper bound of Proposition 1 . For details, see [30] and 32.

Sketch of the proof of Proposition 3 . The idea is simple: equation (5) has a Lyapunov functional $E(w)$ defined in (9) which satisfies

$$
\forall s \geq-\log T\left(x_{0}\right), \frac{d}{d s} E\left(w_{x_{0}}(s)\right)=-\frac{4}{p-1} \int_{-1}^{1}\left(\partial_{s} w_{x_{0}}\right)^{2} \frac{\rho}{1-y^{2}} d y .
$$

From (164), it follows that

$$
\int_{-\log T\left(x_{0}\right)}^{+\infty} \int_{|y|<1}\left(\partial_{s} w\right)^{2} \frac{\rho}{1-y^{2}} d y d s \leq E\left(w_{x_{0}}\left(-\log T\left(x_{0}\right)\right) \leq C_{0} .\right.
$$

From this identity, we see that for any $\epsilon>0$

$$
\int_{s}^{s+1} \int_{|y|<1-\epsilon}\left(\partial_{s} w\right)^{2} \frac{\rho}{1-y^{2}} d y d s \rightarrow 0 \text { as } s \rightarrow+\infty .
$$

This means that $\partial_{s} w \rightarrow 0$, in a certain sense, which means that $w$ would approach the set of stationary solutions. From the lower bound in Proposition $1 w$ cannot approach the zero solutions. Since the set of non-zero solutions of (5) is given by $\left\{0, e^{i \theta} \kappa(\delta, \cdot),|\delta|<1, \theta \in \mathbb{R}\right\}$, we get the conclusion. For the actual proof and for details, see Theorem 2 on page 47 in 33 . 


\section{Appendix B. Explicit solution of the Elliptic EQUATion IN ONE SPACE DIMENSION IN $H^{1}$}

Here we solve equation (27), deriving the well-known KDV solutions. Our aim is to prove (28). Multiplying equation (27) by $W^{\prime}$ and integrating in space we have

$$
\frac{1}{2} W^{\prime 2}-\frac{c}{2} W^{2}+\frac{|W|^{p+1}}{p+1}=K \text {. }
$$

As $W \in H^{1}$, all the terms of the left hand side of (171) are integrable, so $K=0$. We claim that

$$
\exists \xi_{0} \in \mathbb{R}, W^{\prime}\left(\xi_{0}\right)=0,
$$

otherwise, if

$$
\forall \xi \in \mathbb{R}, W^{\prime}(\xi) \neq 0,
$$

$W$ would be monotonic, with limits (in $\overline{\mathbb{R}}$ ) at \pm infinity. Since $W \in L^{2}$, those limits have to be zero, leading to $W \equiv 0$, contradicting (173). Thus (172) holds, and from (171), we see that either

$$
W\left(\xi_{0}\right)=0 \text { or } W\left(\xi_{0}\right)= \pm \kappa_{0},
$$

given in (10). Since we already know two solutions satisfying (172) and (174), namely,

$$
W \equiv 0 \text { or } W(\xi)=\check{k}\left(\xi-\xi_{0}\right),
$$

this concludes the proof of (28).

\section{Appendix C. Basic Properties And some Results}

In the following, we recall some results which we have used in this work. We first give the boundedness for $E$.

Proposition C.1 (Boundedness of the Lyapunov functional for equation (5)). Consider $w(y, s)$ a solution to (5) defined for all $(y, s) \in(-1,1) \times[-\log T,+\infty)$ such that

$$
\left(w, \partial_{s} w\right)(-\log T) \in H^{1} \times L^{2}(-1,1) .
$$

For all $s \geq-\log T$, we have

$$
0 \leq E\left(w(s), \partial_{s} w(s)\right) \leq E\left(w(-\log T), \partial_{s} w(-\log T)\right),
$$

and

$$
\int_{-\log T}^{\infty} \int_{-1}^{1}\left(\partial_{s} w(y, s)\right)^{2} \frac{\rho(y)}{1-y^{2}} d y d s \leq \frac{p-1}{4} E\left(w(-\log T), \partial_{s} w(-\log T)\right) .
$$

Proof. See Antonini and Merle [3].

These following properties have been cited and proved in Section 2 in [33. We first give Hardy-Sobolev identities in the space $\mathcal{H}_{0}$ (8).

Lemma C.2 (A Hardy-Sobolev type identity). For all $h \in \mathcal{H}_{0}$, it holds that

$$
\left(\int_{-1}^{1} h(y)^{2} \frac{\rho(y)}{1-y^{2}} d y\right)^{1 / 2}+\|h\|_{L_{\rho}^{p+1}}+\left\|h\left(1-y^{2}\right)^{\frac{1}{p-1}}\right\|_{L^{\infty}(-1,1)} \leq C\|h\|_{\mathcal{H}_{0}} .
$$


The operator $\mathcal{L}$ introduced in equation (5) has the following properties:

Proposition C.3 (Properties of the operator $\mathcal{L}(6)$ ). The operator $\mathcal{L}$ is self-adjoint in $L_{\rho}^{2}$. For each $n \in \mathbb{N}$, there exists a polynomial $h_{n}$ of degree $n$ such that

$$
\mathcal{L} h_{n}=\gamma_{n} h_{n} \text { where } \gamma_{n}=-n\left(n+\frac{p+3}{p-1}\right) .
$$

The family $\left\{h_{n} \mid n \in \mathbb{N}\right\}$ is orthogonal and spans the whole space $L_{\rho}^{2}$. When $n=0$ and $n=1$, the eigenfunctions are $h_{0}=c_{0}$ and $h_{1}=c_{1} y$ for some positive $c_{0}$ and $c_{1}$, and

$$
\mathcal{L} c_{0}=0, \mathcal{L} c_{1} y=-\frac{2(p+1)}{p-1} c_{1} y
$$

We also claim the following:

Lemma C.4. Consider $u \in L_{\rho}^{2}$ such that $\mathcal{L} u \in L_{\rho}^{2}$ and

$$
\int_{-1}^{1} u(y) \rho(y) d y=0
$$

Then, $\int_{-1}^{1} u \mathcal{L} u \rho d y \leq \gamma_{1} \int u^{2} \rho d y$, where $\gamma_{1}=-2 \frac{p+1}{p-1}$.

Using the Lorentz transform we get a one-dimensional group which keeps invariant equation (5):

Lemma C.5 (The Lorentz transform in similarity variables). Consider $w(y, s) a$ solution of equation (5) defined for all $|y|<1$ and $s \in\left(s_{0}, s_{1}\right)$ for some $s_{0}$ and $s_{1}$ in $\mathbb{R}$, and introduce for any $\delta \in(-1,1)$, the function $W \equiv \mathcal{T}_{\delta}(w)$ defined by

$$
W(Y, S)=\frac{\left(1-\delta^{2}\right)^{\frac{1}{p-1}}}{(1+\delta Y)^{\frac{2}{p-1}}} w(y, s), \text { where } y=\frac{Y+\delta}{1+\delta Y} \text { and } s=S-\log \frac{1+\delta Y}{\sqrt{1-\delta^{2}}} .
$$

Then $W(Y, S)=\mathcal{T}_{\delta}(w)$ is also a solution of (5) defined

$$
\text { for all }|Y|<1 \text { and } S \in\left(s_{0}+\frac{1}{2} \log \frac{1+|\delta|}{1-|\delta|}, s_{1}-\frac{1}{2} \log \frac{1+|\delta|}{1-|\delta|}\right) \text {. }
$$

In the following, we recall from [33] some properties of the transformation $\mathcal{T}_{\delta}$ :

Lemma C.6 (Continuity of $\mathcal{T}_{\delta}$ ). There exists $C_{0}>0$ such that for all $\delta \in(-1,1)$ and $v \in \mathcal{H}_{0}$, we have

(i) (Continuity of $\mathcal{T}_{\delta}$ in $\mathcal{H}_{0}$ )

$$
\frac{1}{C_{0}}\|v\|_{\mathcal{H}_{0}} \leq\left\|\mathcal{T}_{\delta}(v)\right\|_{\mathcal{H}_{0}} \leq C_{0}\|v\|_{\mathcal{H}_{0}} .
$$

(ii) For any $V_{1}$ and $V_{2}$ in $L_{\rho}^{2}$, we have

$$
\int_{-1}^{1} V_{1}(y) V_{2}(y) \rho(y) d y=\int_{-1}^{1} \frac{1-\delta^{2}}{(1-\delta z)^{2}} v_{1}(z) v_{2}(z) \rho(z) d z
$$

where $v_{i}=\mathcal{T}_{-\delta} V_{i}, i \in\{1,2\}$.

Proof. (i) See Lemma 2.8 on page 57 in [33].

(ii) This is a direct consequence of the change of variable (176) and the definition (6) of $\rho(y)$. 


\section{ACKNOWLEDGMENTS}

The author would like to thank the referee for his remarks and suggestions which undoubtedly greatly improved the presentation of our results. The author would also like to thank H. Zaag for useful suggestions during the preparation of this paper.

\section{REFERENCES}

[1] Serge Alinhac, Blowup for nonlinear hyperbolic equations, Progress in Nonlinear Differential Equations and their Applications, 17, Birkhäuser Boston, Inc., Boston, MA, 1995. MR:1339762 (96h:35109)

[2] Serge Alinhac, A minicourse on global existence and blowup of classical solutions to multidimensional quasilinear wave equations, Journées "Équations aux Dérivées Partielles" (Forgesles-Eaux, 2002), Univ. Nantes, Nantes, 2002, pp. Exp. No. I, 33. MR.1968197 (2004b:35227)

[3] Christophe Antonini and Frank Merle, Optimal bounds on positive blow-up solutions for a semilinear wave equation, Internat. Math. Res. Notices 21 (2001), 1141-1167, DOI 10.1155/S107379280100054X. MR1861514(2002h:35027)

[4] Luis A. Caffarelli and Avner Friedman, The blow-up boundary for nonlinear wave equations, Trans. Amer. Math. Soc. 297 (1986), no. 1, 223-241, DOI 10.2307/2000465. MR849476 (87h:35215)

[5] Raphaël Côte and Hatem Zaag, Construction of a multisoliton blowup solution to the semilinear wave equation in one space dimension, Comm. Pure Appl. Math. 66 (2013), no. 10, 1541-1581, DOI 10.1002/cpa.21452. MR3084698

[6] R. Donninger, M. Huang, J. Krieger and W. Schlag, Exotic blowup solutions for the $u^{5}$ focusing wave equation in $\mathbb{R}^{3}$, preprint, arXiv:1212.4718, 2012.

[7] Roland Donninger and Birgit Schörkhuber, Stable blow up dynamics for energy supercritical wave equations, Trans. Amer. Math. Soc. 366 (2014), no. 4, 2167-2189, DOI 10.1090/S00029947-2013-06038-2. MR.3152726

[8] Roland Donninger and Birgit Schörkhuber, Stable self-similar blow up for energy subcritical wave equations, Dyn. Partial Differ. Equ. 9 (2012), no. 1, 63-87, DOI 10.4310/DPDE.2012.v9.n1.a3. MR2909934

[9] Thomas Duyckaerts, Carlos Kenig, and Frank Merle, Universality of blow-up profile for small radial type II blow-up solutions of the energy-critical wave equation, J. Eur. Math. Soc. (JEMS) 13 (2011), no. 3, 533-599, DOI 10.4171/JEMS/261. MR2781926 (2012e:35160)

[10] Thomas Duyckaerts, Carlos Kenig, and Frank Merle, Profiles of bounded radial solutions of the focusing, energy-critical wave equation, Geom. Funct. Anal. 22 (2012), no. 3, 639-698, DOI 10.1007/s00039-012-0174-7. MR2972605

[11] Thomas Duyckaerts, Carlos Kenig, and Frank Merle, Universality of the blow-up profile for small type II blow-up solutions of the energy-critical wave equation: the nonradial case, J. Eur. Math. Soc. (JEMS) 14 (2012), no. 5, 1389-1454, DOI 10.4171/JEMS/336. MR2966655

[12] T. Duyckaerts, C. Kenig, and F. Merle, Classification of radial solutions of the focusing, energy-critical wave equation, Cambridge J. Math. 1 (2013), 75-144.

[13] Thomas Duyckaerts and Frank Merle, Dynamics of threshold solutions for energy-critical wave equation, Int. Math. Res. Pap. IMRP (2008), Art ID rpn002, 67. MR2470571 (2009m:35329)

[14] Stathis Filippas and Frank Merle, Modulation theory for the blowup of vector-valued nonlinear heat equations, J. Differential Equations 116 (1995), no. 1, 119-148, DOI 10.1006/jdeq.1995.1031. MR.1317705 (95m:35087)

[15] J. Ginibre, A. Soffer, and G. Velo, The global Cauchy problem for the critical nonlinear wave equation, J. Funct. Anal. 110 (1992), no. 1, 96-130, DOI 10.1016/0022-1236(92)90044-J. MR.1190421 (94d:35105)

[16] J. Ginibre and G. Velo, Regularity of solutions of critical and subcritical nonlinear wave equations, Nonlinear Anal. 22 (1994), no. 1, 1-19, DOI 10.1016/0362-546X(94)90002-7. MR.1256167 (95g:35129)

[17] M. A. Hamza and H. Zaag, Blow-up behavior for the Klein-Gordon and other perturbed semilinear wave equations, Bull. Sci. Math. 137 (2013), no. 8, 1087-1109, DOI 10.1016/j.bulsci.2013.05.004. MR3130348 
[18] Slim Ibrahim, Nader Masmoudi, and Kenji Nakanishi, Scattering threshold for the focusing nonlinear Klein-Gordon equation, Anal. PDE 4 (2011), no. 3, 405-460, DOI 10.2140/apde.2011.4.405. MR2872122

[19] S. Ibrahim, N. Masmoudi and K. Nakanishi, Threshold solutions in the case of mass-shift for the critical Klein-Gordon equation, Trans. Amer. Math. Soc., to appear, 2014.

[20] Carlos E. Kenig and Frank Merle, Global well-posedness, scattering and blow-up for the energy-critical focusing non-linear wave equation, Acta Math. 201 (2008), no. 2, 147-212, DOI 10.1007/s11511-008-0031-6. MR2461508(2011a:35344)

[21] Carlos E. Kenig and Frank Merle, Radial solutions to energy supercritical wave equations in odd dimensions, Discrete Contin. Dyn. Syst. 31 (2011), no. 4, 1365-1381, DOI 10.3934/dcds.2011.31.1365. MR2836357 (2012j:35256)

[22] Satyanad Kichenassamy and Walter Littman, Blow-up surfaces for nonlinear wave equations. I, Comm. Partial Differential Equations 18 (1993), no. 3-4, 431-452, DOI 10.1080/03605309308820936. MR 1214867 (94f:35087)

[23] Satyanad Kichenassamy and Walter Littman, Blow-up surfaces for nonlinear wave equations. II, Comm. Partial Differential Equations 18 (1993), no. 11, 1869-1899, DOI 10.1080/03605309308820997. MR.1243529(94m:35197)

[24] Rowan Killip, Betsy Stovall, and Monica Visan, Blowup behaviour for the nonlinear KleinGordon equation, Math. Ann. 358 (2014), no. 1-2, 289-350, DOI 10.1007/s00208-013-0960-z. MR 3157999

[25] J. Krieger and W. Schlag, On the focusing critical semi-linear wave equation, Amer. J. Math. 129 (2007), no. 3, 843-913, DOI 10.1353/ajm.2007.0021. MR2325106 (2009f:35231)

[26] Joachim Krieger, Kenji Nakanishi, and Wilhelm Schlag, Global dynamics away from the ground state for the energy-critical nonlinear wave equation, Amer. J. Math. 135 (2013), no. 4, 935-965, DOI 10.1353/ajm.2013.0034. MR.3086065

[27] Joachim Krieger, Kenji Nakanishi, and Wilhelm Schlag, Global dynamics of the nonradial energy-critical wave equation above the ground state energy, Discrete Contin. Dyn. Syst. 33 (2013), no. 6, 2423-2450. MR3007693

[28] Howard A. Levine, Instability and nonexistence of global solutions to nonlinear wave equations of the form $P u_{t t}=-A u+\mathcal{F}(u)$, Trans. Amer. Math. Soc. 192 (1974), 1-21. MR0344697 (49 \#9436)

[29] Hans Lindblad and Christopher D. Sogge, On existence and scattering with minimal regularity for semilinear wave equations, J. Funct. Anal. 130 (1995), no. 2, 357-426, DOI 10.1006/jfan.1995.1075. MR 1335386 (96i:35087)

[30] Frank Merle and Hatem Zaag, Determination of the blow-up rate for the semilinear wave equation, Amer. J. Math. 125 (2003), no. 5, 1147-1164. MR2004432 (2004g:35163)

[31] Frank Merle and Hatem Zaag, Determination of the blow-up rate for a critical semilinear wave equation, Math. Ann. 331 (2005), no. 2, 395-416, DOI 10.1007/s00208-004-0587-1. MR2115461(2005k:35286)

[32] Frank Merle and Hatem Zaag, On growth rate near the blowup surface for semilinear wave equations, Int. Math. Res. Not. 19 (2005), 1127-1155, DOI 10.1155/IMRN.2005.1127. MR2147056(2006h:35183)

[33] Frank Merle and Hatem Zaag, Existence and universality of the blow-up profile for the semilinear wave equation in one space dimension, J. Funct. Anal. 253 (2007), no. 1, 43-121, DOI 10.1016/j.jfa.2007.03.007. MR2362418 (2009b:35288)

[34] Frank Merle and Hatem Zaag, Openness of the set of non-characteristic points and regularity of the blow-up curve for the $1 \mathrm{D}$ semilinear wave equation, Comm. Math. Phys. 282 (2008), no. 1, 55-86, DOI 10.1007/s00220-008-0532-3. MR.2415473 (2010e:35193)

[35] F. Merle and H. Zaag. Isolatedness of characteristic points for a semilinear for a semilinear wave equation in one space dimension. Points caractéristiques à l'explosion pour une équation semilinéaire des ondes. In "Séminaire sur les Équations aux Dérivées Partielles, 2009-2010", pages Exp. No. 11, 10p. École Polytech., Palaiseau, 2010.

[36] Frank Merle and Hatem Zaag, Blow-up behavior outside the origin for a semilinear wave equation in the radial case, Bull. Sci. Math. 135 (2011), no. 4, 353-373, DOI 10.1016/j.bulsci.2011.03.001. MR2799813 (2012i:35247)

[37] Frank Merle and Hatem Zaag, Existence and classification of characteristic points at blow-up for a semilinear wave equation in one space dimension, Amer. J. Math. 134 (2012), no. 3, 581-648, DOI 10.1353/ajm.2012.0021. MR2931219 
[38] Frank Merle and Hatem Zaag, Isolatedness of characteristic points at blowup for a 1dimensional semilinear wave equation, Duke Math. J. 161 (2012), no. 15, 2837-2908, DOI 10.1215/00127094-1902040. MR.2999314

[39] F. Merle and H. Zaag, Dynamics near explicit stationary solutions in similarity variables for solutions of a semilinear wave equation in higher dimensions, Submitted. arXiv:1309.7756, 2013

[40] F. Merle and H. Zaag, On the stability of the notion of non-characteristic point and blow-up profile for semilinear wave equations, Submitted. arXiv:1309.7760, 2013

[41] Kenji Nakanishi and Wilhelm Schlag, Invariant manifolds and dispersive Hamiltonian evolution equations, Zurich Lectures in Advanced Mathematics, European Mathematical Society (EMS), Zürich, 2011. MR2847755 (2012m:37120)

[42] Jalal Shatah and Michael Struwe, Geometric wave equations, Courant Lecture Notes in Mathematics, vol. 2, New York University, Courant Institute of Mathematical Sciences, New York; American Mathematical Society, Providence, RI, 1998. MR.1674843(2000i:35135)

Institut Galilée, Laboratoire Analyse Géometrie et Applications, CNRS-UMR 7539,

Université Paris 13, 99 avenue J.B. Clément 93430, Villetaneuse, France

E-mail address: azaiez@math.univ-paris13.fr 